Publ. Mat. 63 (2019), 361-422

DOI: $10.5565 /$ PUBLMAT6321901

\title{
THE LINEAR NATURE OF PSEUDOWORDS
}

\author{
Jorge Almeida, Alfredo Costa, José Carlos Costa, And \\ MARC ZEITOUN
}

\begin{abstract}
Given a pseudoword over suitable pseudovarieties, we associate to it a labeled linear order determined by the factorizations of the pseudoword. We show that, in the case of the pseudovariety of aperiodic finite semigroups, the pseudoword can be recovered from the labeled linear order.
\end{abstract}

2010 Mathematics Subject Classification: Primary: 20M07, 20M05; Secondary: 06A05, 68Q45, 37B10.

Key words: Relatively free profinite semigroup, aperiodic semigroup, equidivisible semigroup, labeled linear order, pseudoword, pseudovariety.

\section{Contents}

1. Introduction 362

2. Preliminaries 364

3. Equidivisibility and pseudovarieties closed under concatenation

4. The quasi-order of 2-factorizations 368

4.1. Definition and properties 368

4.2. The category of transitions 370

5. The minimum ideal semigroupoid and the $\mathcal{J}$-class associated to a $\sim$-class 372

6. Finitely cancelable semigroups 376

7. Step points and stationary points 379

8. Cluster words 385

9. Stabilizers 389

10. A characterization of the $\mathcal{J}$-class associated to a $\sim$-class $\quad 392$

11. Proof of Theorem $8.6 \quad 394$

12. The effect of multiplication on the quasi-order 403

13. The image of the representation $w \mapsto \mathfrak{L}_{c}(w)$ in the aperiodic case

14. On the cardinality of the set of stationary points 412

14.1. Subshifts 
14.3. Uncountable $<_{\mathcal{R}}$-chains and uncountable sets of stationary points

14.4. About the proof of Theorem 14.7

Acknowledgments

References

\section{Introduction}

Since the publication of Eilenberg's textbook [19], a large body of finite semigroup theory is in fact the theory of pseudovarieties of semigroups. Besides its own mathematical interest, it draws motivation from the connections with computer science through Eilenberg's correspondence between pseudovarieties of semigroups and varieties of regular languages. As pseudovarieties are classes of finite semigroups, only in very special cases do they contain most general members on a given finite set of generators, that is relatively free semigroups, namely semigroups on $n$ generators in the pseudovariety such that every other member of the pseudovariety on $n$ generators is their homomorphic image. To obtain relatively free structures, one needs to step away from finiteness into the more general framework of profinite semigroups, and indeed such a tool has been shown to lead to useful insights and has found many applications $[\mathbf{1}, \mathbf{3}, \mathbf{8}, \mathbf{3 6}, \mathbf{4 7}, 40]$.

As topological semigroups, relatively free profinite semigroups $S$ over a finite alphabet $A$ are generated by $A$, which means that elements of $S$ are arbitrarily well approximated by words in the letters of $A$. Thus, the elements of $S$ may be considered a sort of generalization of words on the alphabet $A$, which are sometimes called pseudowords. Of course, $S$ may satisfy nontrivial identities, which means that different words may represent the same element of $S$, although in the most interesting examples of pseudovarieties, this is not the case. Now, words on the alphabet $A$ may be naturally viewed as $A$-labeled finite linear orders, a perspective that underlies many fruitful connections with finite model theory [45]. For some pseudovarieties, such as $\mathrm{R}$, of all finite $\mathcal{R}$-trivial semigroups, and DA, of all finite semigroups in which the idempotents are the only regular elements, representations of the corresponding finitely generated relatively free profinite semigroups by labeled linear orders have been obtained and significantly applied $[\mathbf{1 0}, \mathbf{3 3}]$. The purpose of this paper is to investigate such a linear nature of pseudowords for pseudovarieties with suitable properties. Our main motivation is to understand pseudowords over the pseudovariety $\mathrm{A}$, of all finite aperiodic semigroups.

The key properties of the pseudovariety $A$ that play a role in this paper are of a combinatorial nature: the corresponding variety of lan- 
guages is closed under concatenation and the cancelability of first and last letters. The first of these properties entails a very useful feature of the corresponding finitely generated relatively free profinite semigroups, namely equidivisibility, which means that different factorizations of the same pseudoword have a common refinement. This condition already forces a linear quasi-order on the factorizations of a given pseudoword, and this is the starting point for the whole paper. The cancelability condition leads to special types of factorizations, which we call step points, to which a letter is naturally associated. The corresponding linear order has interesting order and topological properties, such as being compact for the interval topology. The step points are the isolated points and there are only countably many of them. All other points are called stationary and, in contrast, there may be uncountably many of them. As one goes down in the J-order, one might expect to find more complexity in terms of the number of stationary points. However, it turns out that there is no correlation between the number of stationary points and how low pseudowords fall in the $\mathcal{J}$-order. This is basically due to the fact that the product of pseudowords does not correspond to the mere concatenation of the linear orders associated with the factors, as some fusion may occur near the concatenating point.

Our main result is that the linear order of factorizations with alphabetlabeled step points provides a faithful representation of pseudowords over A. We also obtain a characterization of the partially labeled linear orders that appear in this way, albeit in terms of properties involving finite aperiodic semigroups. A natural goal for future work consists in looking for a characterization of the image of the representation which is independent of such semigroups, as has been done in the case of the pseudovarieties R and DA [10, 33].

While this paper was being written, Gool and Steinberg developed a different approach to pseudowords over A, applying Stone duality and model theory to view them as elementary equivalence classes of labeled linear orders $[\mathbf{2 1}]$. They worked specially with saturated models. In our paper, the models that appear in the image of the representation are not saturated in general. It would be worth developing further research to understand the relationship between the two approaches but this is out of the scope of the present paper.

We also mention the articles [24] and [28], where labeled linear orders were assigned only to a special class of pseudowords, the $\omega$-terms, and were used to solve the word problem for $\omega$-terms in several pseudovarieties, either for the first time, or with new proofs, as in the case of $A$, treated in [24]. 
The paper is organized as follows. After a section of preliminaries, Section 3 introduces the key notion of equidivisible semigroup in the context of relatively free profinite semigroups, with an emphasis on pseudovarieties closed under concatenation. Several results of the paper apply to all such pseudovarieties, but at a certain point our hypothesis restricts to $A$. In the next four sections, we develop more on the tools and the language necessary for the main results. In Section 8, we give our faithful representation of pseudowords over A as labeled linear orders. The following three sections relate to the proof of this representation (the first two of them having independent interest). This is followed by a study of the effect of the multiplication in the image of the representation, and by a characterization of the image. The paper closes with Section 14 where, among other things, it is shown that the ordered set of real numbers can be embedded in the ordered set of the stationary points of a pseudoword over a finitely cancelable pseudovariety containing LSI. This is done via a connection with symbolic dynamics.

\section{Preliminaries}

We assume some familiarity with pseudovarieties of semigroups and relatively free profinite semigroups $[\mathbf{3}, \mathbf{1}, \mathbf{4 0}]$. For the reader's convenience, some notation and terminology is presented here. The following is a list of some of the pseudovarieties we will be working with:

- I: all trivial semigroups;

- S: all finite semigroups;

- A: all finite aperiodic semigroups;

- N: all finite nilpotent semigroups;

- D: all finite semigroups in which the idempotents are right zeros;

- LSI: all finite local semilattices.

In the whole paper, $A$ denotes a finite alphabet. Let $\mathrm{V}$ be a pseudovariety of semigroups. The free pro- $\mathrm{V}$ semigroup generated by $A$ is denoted $\bar{\Omega}_{A} \mathrm{~V}$. Its elements are pseudowords over $\mathrm{V}$. When $\mathrm{V} \neq \mathrm{I}$, as the associated generating mapping $A \rightarrow \bar{\Omega}_{A} \mathrm{~V}$ is injective, one considers $A$ to be contained in $\bar{\Omega}_{A} \mathrm{~V}$. If $\varphi: A \rightarrow S$ is a generating mapping of a pro- $\mathrm{V}$ semigroup, then we denote by $\varphi_{\mathrm{V}}$ the unique continuous homomorphism $\bar{\Omega}_{A} \mathrm{~V} \rightarrow S$ extending $\varphi$.

If $\mathrm{V}$ contains $\mathrm{N}$, then the subsemigroup of $\bar{\Omega}_{A} \mathrm{~V}$ generated by $A$ is isomorphic to $A^{+}$and its elements are the isolated points of $\bar{\Omega}_{A} \mathrm{~V}$, in view of which $A^{+}$is considered to be contained in $\bar{\Omega}_{A} \mathrm{~V}$, and the elements of $A^{+}$and $\bar{\Omega}_{A} \vee \backslash A^{+}$are respectively called the finite and infinite pseudowords over $\mathrm{V}$. 
By a topological semigroup, we mean a semigroup endowed with a topology that makes the semigroup multiplication continuous. Unlike some authors, we require that a compact space be Hausdorff. By a compact semigroup, we mean a compact topological semigroup. See [14].

We denote by $S^{I}$ the monoid obtained from the semigroup $S$ by adjoining to $S$ an element denoted by 1 which acts as the identity. Every semigroup homomorphism $\varphi: S \rightarrow T$ is extended to a semigroup homomorphism $S^{I} \rightarrow T^{I}$, also denoted $\varphi$, such that $\varphi(1)=1$. If $S$ is a topological semigroup, then $S^{I}$ is viewed as a topological monoid whose topology is the sum of the topological spaces $S$ and $\{1\}$, whence 1 is an isolated point of $S^{I}$.

We use the standard notation for Green's relations and its quasi-orders on a semigroup $S$. Hence, $s \leq_{\mathcal{R}} t, s \leq_{\mathcal{L}} t$, and $s \leq_{\mathcal{J}} t$ respectively mean $s \in t S^{I}, s \in S^{I} t$, and $s \in S^{I} t S^{I} ; \mathcal{R}, \mathcal{L}, \mathcal{J}$ are the associated equivalence relations, $\mathcal{D}=\mathcal{R} \vee \mathcal{L}$, and $\mathcal{H}=\mathcal{R} \cap \mathcal{L}$.

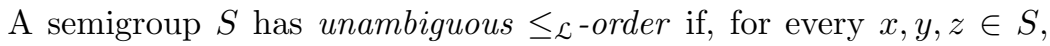
$x \leq_{\mathcal{L}} y$ and $x \leq_{\mathcal{L}} z$ implies $y \leq_{\mathcal{L}} z$ or $z \leq_{\mathcal{L}} y$. One also has the dual notion of unambiguous $\leq_{\mathcal{R}}$-order. An unambiguous semigroup is a semigroup with unambiguous $\leq_{\mathcal{R}}$-order and unambiguous $\leq_{\mathcal{L}}$-order. The next proposition is an important tool to show one of our main results.

Proposition 2.1. Let $A$ be a finite alphabet. Let $u, v \in \bar{\Omega}_{A} \mathrm{~A}$. Then $u=v$ if and only if $\varphi_{\mathrm{A}}(u)=\varphi_{\mathrm{A}}(v)$ for every mapping $\varphi$ from $A$ onto an unambiguous finite aperiodic semigroup.

Proof: The "only if" direction of the statement is immediate. To establish the "if" direction, it suffices to show that points of $\bar{\Omega}_{A} \mathrm{~A}$, that may be separated by continuous homomorphisms into finite aperiodic semigroups, may also be separated by such mappings into finite unambiguous aperiodic semigroups.

It is well known that every $A$-generated finite aperiodic semigroup is the image under a homomorphism respecting generators of an unambiguous $A$-generated finite aperiodic semigroup, namely its Birget-Rhodes expansion (also called iterated Rhodes expansion), cut down to the set of generators $A[\mathbf{1 2}, \mathbf{2 2}]$. Since pairs of distinct points of $\bar{\Omega}_{A} \mathrm{~A}$ may be separated by continuous homomorphisms into finite aperiodic semigroups, the result follows.

\section{Equidivisibility and pseudovarieties closed under concatenation}

A language $L \subseteq A^{+}$is said to be $\mathrm{V}$-recognizable if there is a homomorphism $\varphi: A^{+} \rightarrow S$ into a semigroup $S$ from $\vee$ such that $L=\varphi^{-1} \varphi(L)$. 
We say that a pseudovariety $\mathrm{V}$ of semigroups is closed under concatenation if, for every finite alphabet $A$, whenever $L$ and $K$ are $\mathrm{V}$-recognizable languages of $A^{+}$, the set $L K$ is also a $\mathrm{V}$-recognizable language of $A^{+}$. We use $(m)$ to denote the Mal'cev product of pseudovarieties.

Theorem 3.1. The following conditions are equivalent for a pseudovariety $\mathrm{V}$ of semigroups:

(a) $\mathrm{V}$ is closed under concatenation;

(b) $\mathrm{A}$ (m) $\mathrm{V}=\mathrm{V}$;

(c) $\mathrm{V}$ contains $\mathrm{N}$ and the multiplication in $\bar{\Omega}_{A} \mathrm{~V}$ is an open mapping for every finite alphabet $A$.

The equivalence $(\mathrm{a}) \Leftrightarrow(\mathrm{c})$ in Theorem 3.1 is from [4, Lemma 2.3]. The difficult part of the theorem is the equivalence $(a) \Leftrightarrow(b)$, which is a particular case of a more general result established by Chaubard, Pin, and Straubing [15]. The latter, in turn, extends an earlier result of Straubing [44], establishing that a nontrivial pseudovariety $\mathrm{V}$ of monoids satisfies $\mathrm{A}(m \mathrm{~V}=\mathrm{V}$ if and only if, for every finite alphabet $A$, whenever $L$ and $K$ are $\mathrm{V}$-recognizable languages of $A^{*}$, the set $L K$ is also a $\mathrm{V}$-recognizable language of $A^{*}$. In the case of semigroups, the absence in Theorem 3.1 of reference to the pseudovariety I of trivial semigroups is not surprising if we take into account that $A^{+}$is I-recognizable but $A^{+} A^{+}$is not, where we view these languages as languages of $A^{+}$.

Schützenberger $[\mathbf{4 3}]$ proved that a language over a finite alphabet is A-recognizable if and only if it is star-free, in the sense that it can be obtained from finite languages by using only finite Boolean operations and concatenation. In particular, it follows that $A$ is closed under concatenation. As important classes of examples of pseudovarieties closed under concatenation that include $\mathrm{A}$, one has the complexity pseudovarieties $\mathrm{C}_{n}$ (cf. [40, Definition 4.3.10]) and every pseudovariety $\overline{\mathrm{H}}$ formed by the finite semigroups whose subgroups belong to the pseudovariety of groups $\mathrm{H}$.

Combined with Theorem 3.1, the next lemma, which will be quite useful in the sequel, provides yet another characterization of the pseudovarieties closed under concatenation. A weaker version of the direct implication was proved in [4, Lemma 2.5]. In the special case of relatively free profinite semigroups over pseudovarieties $\mathrm{V}$ such that $\mathrm{V}=$ A $m \mathrm{~V}$, condition (b) of Lemma 3.2 below was established in the proof of [23, Lemma 4.3], which in turn provides a proof of $(b) \Rightarrow(c)$ in Theorem 3.1 . 
Lemma 3.2. Let $S$ be a topological semigroup whose topology is defined by a metric. The following conditions are equivalent:

(a) The multiplication in $S$ is an open mapping.

(b) For every $u, v \in S$, if $\left(w_{n}\right)_{n}$ is a sequence of elements of $S$ converging to $u v$, then there are sequences $\left(u_{n}\right)_{n}$ and $\left(v_{n}\right)_{n}$ of elements of $S^{I}$ such that $w_{n}=u_{n} v_{n}, \lim u_{n}=u$, and $\lim v_{n}=v$.

Proof: Consider a metric $d$ inducing the topology of $S$. We denote by $B(t, \varepsilon)$ the open ball in $S$ with center $t$ and radius $\varepsilon$.

$(\mathrm{a}) \Rightarrow(\mathrm{b})$ : Let $k$ be a positive integer. Since the multiplication is an open mapping, the set $B\left(u, \frac{1}{k}\right) B\left(v, \frac{1}{k}\right)$ is an open neighbourhood of $u v$. Hence there is $p_{k}$ such that $w_{n} \in B\left(u, \frac{1}{k}\right) B\left(v, \frac{1}{k}\right)$ if $n \geq p_{k}$. Let $n_{k}$ be the strictly increasing sequence recursively defined by $n_{1}=p_{1}$ and $n_{k}=$ $\max \left\{n_{k-1}+1, p_{k}\right\}$ whenever $k>1$. Then there are sequences $\left(u_{n}\right)_{n}$ and $\left(v_{n}\right)_{n}$ satisfying the following conditions: if $n_{k} \leq n<n_{k+1}$ then $u_{n} \in B\left(u, \frac{1}{k}\right), v_{n} \in B\left(v, \frac{1}{k}\right)$, and $w_{n}=u_{n} v_{n}$; and if $n<n_{1}$ then $u_{n}=1$ and $v_{n}=w_{n}$. The pair of sequences $\left(u_{n}\right)_{n}$ and $\left(v_{n}\right)_{n}$ satisfies condition (b).

(b) $\Rightarrow(\mathrm{a})$ : We want to prove that $B(s, \varepsilon) B(t, \varepsilon)$ is open, for every $s, t \in S$ and $\varepsilon>0$. Let $\left(w_{n}\right)_{n}$ be a sequence of elements of $S$ converging to an element of $B(s, \varepsilon) B(t, \varepsilon)$. Let $u \in B(s, \varepsilon)$ and $v \in B(t, \varepsilon)$ be such that $\lim w_{n}=u v$. Take sequences $\left(u_{n}\right)_{n}$ and $\left(v_{n}\right)_{n}$ as in the statement of condition (b). There is $N$ such that $d\left(u_{n}, u\right)<\varepsilon-d(u, s)$ for all $n \geq$ $N$. Then $d\left(u_{n}, s\right) \leq d\left(u_{n}, u\right)+d(u, s)<\varepsilon$ for all $n \geq N$. Similarly, $d\left(v_{n}, t\right)<\varepsilon$ for all sufficiently large $n$. Therefore, since $w_{n}=u_{n} v_{n}$, we have $w_{n} \in B(s, \varepsilon) B(t, \varepsilon)$ for all sufficiently large $n$, which proves that $B(s, \varepsilon) B(t, \varepsilon)$ is open.

A semigroup $S$ is said to be equidivisible $[32, \mathbf{2 9}]$ if, for every equality of the form $x y=u v$, with $x, y, u, v \in S$, there exists $t \in S^{I}$ such that, either $x t=u$ and $y=t v$, or $x=u t$ and $t y=v$. Clearly, free semigroups and groups are equidivisible. Moreover, all completely simple semigroups are equidivisible. Actually, a semigroup $S$ is completely simple if and only if, for every $x, y, u, v \in S$ such that $x y=u v$, there are $t, s \in S$ such that $x t=u, y=t v, x=u s$, and $s y=v[\mathbf{3 2}]$. Note that every equidivisible semigroup is unambiguous. The converse is not true: for instance, free bands are unambiguous, which follows easily from the solution of the word problem for free bands (see, for instance $[\mathbf{1}$, Section 5.4]) but not equidivisible for more than one free generator since, if $a, b$ are two distinct free generators in a free band then, for $x=a$, $y=b, u=v=a b$, we have $x y=u v$, yet $y>_{\mathcal{L}} v$ and $x>_{\mathcal{R}} u$. More 
generally, it is shown in [39, Section 15] that, if $\mathrm{V}$ is a pseudovariety of semigroups such that $\mathrm{V}=\mathrm{RB}(\mathrm{m} \mathrm{V}$, where RB denotes the pseudovariety of finite rectangular bands, then $\bar{\Omega}_{A} \mathrm{~V}$ is unambiguous for every finite alphabet $A$.

Let us say that a pseudovariety of semigroups $\mathrm{V}$ is equidivisible if $\bar{\Omega}_{A} \mathrm{~V}$ is equidivisible for every finite alphabet $A$. This property is implicitly but not explicitly established in $[\mathbf{2 3}]$ for pseudovarieties of the form $\mathrm{A} \cap \mathrm{V} \mathrm{V}$. The following result was proved by the first two authors $[\mathbf{6}]$, where LI denotes the pseudovariety of all finite locally trivial semigroups and CS the pseudovariety of all finite completely simple semigroups.

Theorem 3.3. A pseudovariety of semigroups $\mathrm{V}$ is equidivisible if and only if $\mathrm{V}=\mathrm{LI} \rightarrow \mathrm{V}$ or $\mathrm{V} \subseteq \mathrm{CS}$.

In particular, every pseudovariety closed under concatenation is equidivisible. Many of our results below are formulated not in terms of pseudovarieties but more abstractly for free profinite semigroups with suitable properties, which are satisfied for free profinite semigroups over pseudovarieties that are closed under concatenation or, sometimes, more generally, equidivisible.

\section{The quasi-order of 2-factorizations}

By a quasi-order on a set we mean a reflexive transitive relation. In case the relation is additionally anti-symmetric, the quasi-order is called a partial order. A quasi-ordering $(X, \leq)$, in the sense of a set $X$ with a quasi-order $\leq$, is said to be total, or linear if $x \leq y$ or $y \leq x$, for every $x, y \in X$.

4.1. Definition and properties. Let $S$ be a semigroup. A 2-factorization of $s \in S$ is a pair $(u, v)$ of elements of $S^{I}$ such that $s=u v$. We denote the set of 2-factorizations of $s$ by $\mathfrak{F}(s)$. We introduce in $\mathfrak{F}(s)$ a relation $\leq$ defined by $(u, v) \leq\left(u^{\prime}, v^{\prime}\right)$ if there exists $t \in S^{I}$ such that $u t=u^{\prime}$ and $v=t v^{\prime}$, in which case we say that $t$ is a transition from $(u, v)$ to $\left(u^{\prime}, v^{\prime}\right)$. The relation $\leq$ is a quasi-order. Concerning transitivity, we have more precisely that if $t$ is a transition from $(u, v)$ to $\left(u^{\prime}, v^{\prime}\right)$ and $t^{\prime}$ is a transition from $\left(u^{\prime}, v^{\prime}\right)$ to $\left(u^{\prime \prime}, v^{\prime \prime}\right)$, then $t t^{\prime}$ is a transition from $(u, v)$ to $\left(u^{\prime \prime}, v^{\prime \prime}\right)$.

Given a quasi-order $\leq$ on a set $P$, we denote by $\sim$ the equivalence relation on $P$ induced by $\leq$ and we write $p<q$ if $p \leq q$ but not $p \sim q$. Denote by $\prec$ the relation on $P$ such that $p \prec q$ if and only if $q$ is a successor of $p$ (equivalently, $p$ is a predecessor of $q$ ), that is, $p \prec q$ if and only if $p<q$ and $p \leq r \leq q \Rightarrow(r \sim p \vee r \sim q)$. 
For every element $s$ of a semigroup $S$, the quotient set $\mathfrak{F}(s) / \sim$ is denoted $\mathfrak{L}(s)$. We denote the quotient mapping $\mathfrak{F}(s) \rightarrow \mathfrak{L}(s)$ by $\chi$. The partial order on $\mathfrak{L}(s)$ induced by the quasi-order $\leq$ on $\mathfrak{F}(s)$ is also denoted by $\leq$. For $p, q \in \mathfrak{L}(s)$, we also write $p \prec q$ if $p$ is a predecessor of $q$. Sometimes we will also consider the unions $\mathfrak{F}(S)=\bigcup_{s \in S} \mathfrak{F}(s)$ and $\mathfrak{L}(S)=\bigcup_{s \in S} \mathfrak{L}(s)$.

The following result is immediate.

Lemma 4.1. A semigroup $S$ is equidivisible if and only if $\mathfrak{L}(s)$ is linearly ordered for every $s \in S$.

The previous lemma is the departing point motivating this paper. For a good supporting reference on the theory of linear orderings, see [42].

We proceed to extract from topological assumptions on $S$ some consequences on the quasi-order of 2 -factorizations. In what follows, $\mathfrak{F}(s)$ is viewed as a topological subspace of $S^{I} \times S^{I}$.

Lemma 4.2. If $S$ is a compact semigroup, then, for every $s \in S$, the quasi-order $\leq$ on $\mathfrak{F}(s)$ is a closed subset of $\mathfrak{F}(s) \times \mathfrak{F}(s)$.

Proof: Suppose $\left(p_{i}, q_{i}\right)_{i \in I}$ is a convergent net of elements of $\mathfrak{F}(s) \times \mathfrak{F}(s)$ with limit $(p, q)$ and such that $p_{i} \leq q_{i}$ for every $i \in I$. Then, for each $i \in I$, there is $t_{i} \in S^{I}$ making a transition from $p_{i}$ to $q_{i}$. Since $S^{I}$ is compact, the net $\left(t_{i}\right)_{i \in I}$ has a subnet converging to some $t \in S^{I}$. Then, by continuity of multiplication on $S^{I}$, one deduces that indeed $p \leq q$, with $t$ being a transition from $p$ to $q$.

We shall denote the proper open intervals of a quasi-ordered set $P$ by ]$\leftarrow, p[=\{r \in P: r<p\}] p,, \rightarrow[=\{r \in P: p<r\}] p,, q[=] p, \rightarrow[\cap] \leftarrow, q[$, for every $p, q \in P$. Considering the relation $\leq$, we also have the intervals of the form $] \leftarrow, p]=\{r \in P: r \leq p\},[p, \rightarrow[=\{r \in P: p \leq r\}$, and so on. Recall that the order topology of a linearly ordered set $P$ is the topology with subbase the sets of the form $] \leftarrow, p[$ and $] p, \rightarrow[$. In particular, we consider the order topology on $\mathfrak{L}(s)$.

The underlying order part of the following result is probably well known but we have not been able to locate it in the literature.

Proposition 4.3. Let $S$ be a compact equidivisible semigroup. For every $s \in S$, the mapping $\chi: \mathfrak{F}(s) \rightarrow \mathfrak{L}(s)$ is continuous.

Proof: It is sufficient to show that the sets of both forms $\left.\left.\chi^{-1}(] \leftarrow, q\right]\right)$ and $\chi^{-1}([q, \rightarrow[)$ are closed. By duality, we are actually reduced to show that $\chi^{-1}\left(\left[q, \rightarrow[)\right.\right.$ is closed. Consider a net $\left(r_{i}\right)_{i \in I}$ of elements of $\left.\left.\chi^{-1}(] \leftarrow, q\right]\right)$, 
converging to some $r \in \mathfrak{F}(s)$. Let $\hat{q} \in \chi^{-1}(q)$. Then $r_{i} \leq \hat{q}$ for every $i \in I$. It follows from Lemma 4.2 that $r \leq \hat{q}$, that is, $\left.\left.r \in \chi^{-1}(] \leftarrow, q\right]\right)$, showing that $\left.\left.\chi^{-1}(] \leftarrow, q\right]\right)$ is closed.

Corollary 4.4. Let $S$ be a compact equidivisible semigroup. Then, for every $s \in S$, the order topology of $\mathfrak{L}(s)$ is compact. Moreover, if the space $S$ is metrizable, then the space $\mathfrak{L}(s)$ is also metrizable and the set of isolated points of $\mathfrak{L}(s)$ is countable.

Proof: Since $S$ is compact, $\mathfrak{F}(s)$ is compact, being the preimage in $S^{I} \times S^{I}$ under multiplication of the closed set $\{s\}$, it is a closed subset of a compact space, whence compact. Since $\mathfrak{L}(s)$ is clearly Hausdorff, it follows from Proposition 4.3 that $\mathfrak{L}(s)$ is compact.

Suppose that $S$ is metrizable. Then $\mathfrak{F}(s)$ is metrizable, being a subspace of a product of two metrizable spaces. Since the continuous image of a compact metric space in a Hausdorff space is metrizable (cf. [48, Corollary 23.2]), it also follows from Proposition 4.3 that $\mathfrak{L}(s)$ is metrizable. As a compact metrizable space, $\mathfrak{L}(s)$ has a dense countable subset. Since isolated points belong to every dense subset, they form a countable set.

Recall that a linearly ordered set $L$ is said to be complete if every subset of $L$ which is bounded above has a least upper bound (i.e., a supremum) or, equivalently, if every subset of $L$ which is bounded below has a greatest lower bound (i.e., an infimum) [42, Section 2.4].

Proposition 4.5. Suppose $S$ is a compact equidivisible semigroup. Then the linearly ordered set $\mathfrak{L}(s)$ is complete.

Proof: As $S$ is equidivisible, we know by Lemma 4.1 that $\mathfrak{L}(s)$ is a linear order, whence in particular it is a semilattice. By Corollary $4.4, \mathfrak{L}(s)$ is also compact. By [26, Corollary VII.1.5], it follows that $\mathfrak{L}(s)$ is complete.

4.2. The category of transitions. A directed graph with vertex set $V$ and edge set $E$, which are assumed to be disjoint, is given by mappings $\alpha, \omega: E \rightarrow V$ assigning to each edge $s$ its source $\alpha(s)$ and its target $\omega(s)$. A semigroupoid is a directed graph, with a nonempty set of edges, endowed with a partial associative binary operation on the set of its edges such that if $s$ and $t$ are edges, then $s t$ is defined if and only if $\omega(s)=\alpha(t)$, in which case $\alpha(s t)=\alpha(s)$ and $\omega(s t)=\omega(t)$.

Semigroupoids can be viewed as generalizations of semigroups, which in turn can be viewed as one-vertex semigroupoids. In particular, Green's relations generalize straightforwardly to Green's relations between the 
edges in semigroupoids. For instance, in a semigroupoid $S, s \leq_{\mathcal{J}} t$ means that the edge $t$ is a factor of the edge $s$ and $s \mathcal{J} t$ means that $s \leq_{\mathrm{g}} t$ and $t \leq_{\text {f }} s$. A subsemigroupoid of the semigroupoid $S$ is a subgraph $T$ of $S$, with a nonempty set of edges, such that $s, t \in T$ implies $s t \in T$ whenever $\omega(s)=\alpha(t)$. Also, an ideal of a semigroupoid $S$ is a subsemigroupoid $I$ of $S$ such that for every $t \in I$ and every $s \in S, \omega(s)=\alpha(t)$ implies $s t \in I$, and $\omega(t)=\alpha(s)$ implies $t s \in I$.

A category is a semigroupoid such that, for each vertex $v$, there is a loop $1_{v}$ at $v$ satisfying $1_{v} s=s$ and $t 1_{v}=t$ for every edge $s$ starting in $v$ and every edge $t$ ending in $v$. This coincides with the notion of small category from Category Theory, except that we compose in the opposite direction. In doing so, we are following a common convention in Semigroup Theory, see for example [46].

If the sets of edges and vertices of a semigroupoid are both endowed with compact topologies, for which the semigroupoid operation and the mappings $\alpha$ and $\omega$ are continuous, then the semigroupoid is said to be compact.

Let $S$ be an arbitrary semigroup. To each $s \in S$, we associate a category $\mathcal{T}(s)$, the category of transitions for $s$, as follows:

(a) the set of vertices of $\mathcal{T}(s)$ is $\mathfrak{F}(s)$;

(b) we have an edge $(u, v, t, x, y)$ from $(u, v)$ to $(x, y)$, which we may denote $(u, v) \stackrel{t}{\rightarrow}(x, y)$, if $t$ is a transition from $(u, v)$ to $(x, y)$ (thus implying $(u, v) \leq(x, y))$; we say that $t$ is the label of the edge;

(c) multiplication of consecutive edges is done by multiplying their labels, that is, the product of $\left(u_{1}, v_{1}\right) \stackrel{t_{1}}{\rightarrow}\left(u_{2}, v_{2}\right)$ an $\left(u_{2}, v_{2}\right) \stackrel{t_{2}}{\longrightarrow}$ $\left(u_{3}, v_{3}\right)$ is $\left(u_{1}, v_{1}\right) \stackrel{t_{1} t_{2}}{\longrightarrow}\left(u_{3}, v_{3}\right)$.

Note that the sets of vertices of the strongly connected components of the category $\mathcal{T}(s)$ are precisely the $\sim$-classes of $\mathfrak{F}(s)$.

The category of transitions for $S$, denoted $\mathcal{T}(S)$, is the coproduct category $\bigcup_{s \in S} \mathcal{T}(s)$. This natural construction appears elsewhere in the literature. Rhodes and Tilson introduced in [41] the kernel category of a relational morphism of semigroups, of which $\mathcal{T}(S)$ is an example: $\mathcal{T}(S)$ is the kernel category of the identity on $S$. More precisely, in [41] one works with monoids, but an immediate translation is provided via the mapping $S \mapsto S^{I}$, as done in [40] in situations where one deals with the semigroup counterpart of some construction on monoids. We also mention that in $[\mathbf{4 1}]$ the kernel category of the identity on a monoid is called the two-sided Cayley category.

We denote by $\Lambda$ the faithful functor $\mathcal{T}(S) \rightarrow S^{I}$ mapping each edge $(u, v, t, x, y)$ to $t$. We say that $\Lambda$ is the labeling functor associated to $\mathcal{T}(S)$. 
We remark that if $S$ is a compact semigroup, then $\mathcal{T}(S)$ is a compact category, with the vertex and edge sets respectively endowed with the subspace topology of $\left(S^{I}\right)^{2}$ and of $\left(S^{I}\right)^{5}$. Note that $\Lambda$ is continuous.

Suppose that in $\mathfrak{L}(s)$ we have $p \leq q$. An element $t \in S^{I}$ will be called a transition from $p$ to $q$ if $t$ is a transition from an element of $p$ to an element of $q$, in which case we use the notation $p \stackrel{t}{\rightarrow} q$.

For future reference, it is convenient to register the following remark, concerning the relationship between $\mathcal{T}(u)$ and $\mathcal{T}(u v)$.

Remark 4.6. Let $u, v$ be elements of a semigroup $S$. If $(\alpha, \beta) \stackrel{t}{\rightarrow}(\gamma, \delta)$ is an edge of $\mathcal{T}(u)$, then $(\alpha, \beta v) \stackrel{t}{\rightarrow}(\gamma, \delta v)$ is an edge of $\mathcal{T}(u v)$.

This remark is applied in the proof of the following lemma, which in turn will later be used in the proof of Theorem 10.1.

Lemma 4.7. Let $S$ be an equidivisible semigroup. Consider two edges $\sigma$ and $\tau$ of $\mathcal{T}(S)$ with the same target and such that $\alpha(\sigma)<\alpha(\tau)$. Then the label of $\sigma$ is a suffix of the label of $\tau$.

Proof: Let $\sigma$ be the edge $(\beta, \gamma) \stackrel{t}{\rightarrow}(\varphi, \psi)$ and $\tau$ be the edge $(\delta, \varepsilon) \stackrel{z}{\rightarrow}$ $(\varphi, \psi)$, with $(\beta, \gamma)<(\delta, \varepsilon)$. The following equalities hold: $\varphi=\beta t=\delta z$, $\gamma=t \psi$, and $\varepsilon=z \psi$. From the equality $\beta t=\delta z$ and by equidivisibility, we deduce that if $z$ is not a suffix of $t$, then there exists $s \in S$ such that $\delta s=\beta$ and $s t=z$, that is, we have an edge $(\delta, z) \stackrel{s}{\rightarrow}(\beta, t)$ in $\mathcal{T}(\varphi)$. By Remark 4.6, there is an edge $(\delta, \varepsilon) \stackrel{s}{\rightarrow}(\beta, \gamma)$ in $\mathcal{T}(S)$, which contradicts the hypothesis that $(\beta, \gamma)<(\delta, \varepsilon)$. Hence $z$ is a suffix of $t$.

\section{The minimum ideal semigroupoid and the $\mathcal{J}$-class associated to a $\sim$-class}

In a strongly connected compact semigroupoid $C$, there is an underlying minimum ideal semigroupoid $K(C)$ which may be defined as follows. Consider any vertex $v$ of $C$ and the local semigroup $C_{v}$ of $C$ at $v$, that is, the semigroup formed by the loops at $v$. Then $C_{v}$ is a compact semigroup, and therefore it has a minimum ideal $K_{v}$. Let $K(C)$ be the subsemigroupoid of $C$ with the same set of vertices as $C$ and whose edges are those edges of $C$ which admit some (and therefore every) element of $K_{v}$ as a factor. The next lemma is folklore.

Lemma 5.1. If $C$ is a strongly connected compact semigroupoid, then $K(C)$ is a closed ideal of $C$ whose definition does not depend on the choice of $v$. Moreover, the edges in $K(C)$ are J-equivalent, more precisely they are J-below every edge of $C$. 
Let $(u, v) \in \mathfrak{F}(S)$. An element $z \in S^{I}$ stabilizes $(u, v)$ if $z$ labels a loop of $\mathcal{T}(S)$ at $(u, v)$. Note that the set $M_{(u, v)}$ of stabilizers of $(u, v)$ is a monoid and that $M_{(u, v)}$ is the isomorphic image, under the labeling functor $\Lambda$, of the local monoid of $\mathcal{T}(S)$ at $(u, v)$.

Assume $S$ is a compact semigroup. For $p \in \mathfrak{L}(S)$, let $\mathcal{T}_{p}$ be the strongly connected component of $\mathcal{T}(S)$ whose vertices are the elements of $p$. We denote by $\mathcal{K}_{p}$ the minimum ideal semigroupoid $K\left(\mathcal{T}_{p}\right)$. Since $\Lambda: \mathcal{T}(S) \rightarrow S^{I}$ is a (continuous) functor, where $S^{I}$ is viewed as the set of edges of a single vertex semigroupoid, in view of Lemma 5.1 the set of labels of edges in $\mathcal{K}_{p}$ is contained in a single $\mathcal{J}$-class of $S^{I}$, which we denote $J_{p}$. For every $(u, v) \in p$, the minimum ideal of $M_{(u, v)}$, which we denote $I_{(u, v)}$, is the image under $\Lambda$ of the minimum ideal of the local monoid of $\mathcal{T}(S)$ at $(u, v)$, whence $I_{(u, v)} \subseteq J_{p}$. Note that $J_{p}$ is regular, since $I_{(u, v)}$ is itself regular. The set $J_{p}$ can also be characterized as the set of $\mathcal{J}$-minimum transitions from $p$ to itself, as seen in the next lemma.

Lemma 5.2. Let $S$ be a compact semigroup, and let $p \in \mathfrak{L}(S)$. Then $t$ is a transition from $p$ to $p$ if and only if $t$ is a factor of the elements of $J_{p}$.

Proof: Let $(u, v) \stackrel{t}{\rightarrow}(x, y)$ be a transition between elements of $p$. Since $(u, v) \sim(x, y)$, there is a transition $(x, y) \stackrel{s}{\rightarrow}(u, v)$. The loop $(u, v) \stackrel{t s}{\longrightarrow}$ $(u, v)$ is a factor of every element $\varepsilon$ in the minimum ideal of the local monoid at $(u, v)$. Therefore, $t s$ is a factor of $\Lambda(\varepsilon) \in J_{p}$.

Conversely, suppose that $t$ is factor of the elements of $J_{p}$. Then there is a loop $(u, v) \stackrel{z}{\rightarrow}(u, v)$ in $\mathcal{K}_{p}$ such that $z=x t y$ for some $x, y \in S^{I}$. In $\mathcal{T}(S)$ we have the following path: $(u, v) \stackrel{x}{\rightarrow}(u x, t y v) \stackrel{t}{\rightarrow}(u x t, y v) \stackrel{y}{\rightarrow}(u, v)$. Therefore, $(u x, t y v) \stackrel{t}{\rightarrow}(u x t, y v)$ is an edge of $\mathcal{T}_{p}$.

We next give some results that further highlight the role of idempotent stabilizers of 2-factorizations of elements of $S$, specially those idempotents in a $\mathcal{J}$-class of the form $J_{p}$.

Recall that a semigroup is stable if $\mathcal{J} \cap \leq_{\mathcal{L}}=\mathcal{L}$ and $\mathcal{J} \cap \leq_{\mathcal{R}}=\mathcal{R}$. In particular, any compact semigroup is stable, see for instance [40].

Lemma 5.3. Let $S$ be a stable unambiguous semigroup. Let $e, f$ be idempotents stabilizing an element $(u, v)$ of $\mathfrak{F}(S)$. If e $\mathcal{J} f$ then $e=f$.

Proof: The hypothesis gives $u e=u=u f$ and $e v=v=f v$. Since $S$ is unambiguous, from $u e=u f$ we get $e \leq_{\mathcal{L}} f$ or $f \leq_{\mathcal{L}} e$. By stability, as $e \mathcal{J} f$, it follows that $e \mathcal{L} f$. Dually, from $e v=f v$ we get $e \mathcal{R} f$. Hence $e=f$. 
Corollary 5.4. Let $S$ be a compact unambiguous semigroup, $p \in \mathfrak{L}(S)$, and $(u, v),(x, y) \in p$. The edge $(u, v) \stackrel{t}{\rightarrow}(x, y)$ of $\mathcal{T}(S)$ belongs to $\mathcal{K}_{p}$ if and only if $t \in J_{p}$.

Proof: The "only if" part holds by definition of $J_{p}$. Conversely, suppose that $t \in J_{p}$. Denote by $\varepsilon$ the edge $(u, v) \stackrel{t}{\rightarrow}(x, y)$. As $J_{p}$ is regular, there is an idempotent $e \in J_{p}$ such that $t=e t$. Since $t$ is a prefix of $v$, we have $v=e v$, thus we may consider the edges $(u, v) \stackrel{e}{\rightarrow}(u e, v)$, $(u e, v) \stackrel{e}{\rightarrow}(u e, v)$, and $(u e, v) \stackrel{t}{\rightarrow}(x, y)$, respectively denoted by $\alpha, \beta$, and $\gamma$. Observe that $\varepsilon=\alpha \beta \gamma$, and so it suffices to show that the loop $\beta$ belongs to $\mathcal{K}_{p}$. The ideal $\mathcal{K}_{p}$ contains the minimum ideal of the local monoid of $\mathcal{T}(S)$ at $(u e, v)$. The latter contains an idempotent, of the form $(u e, v) \stackrel{f}{\rightarrow}(u e, v)$ for some $f \in J_{p}$. But $f=e$ by Lemma 5.3 and therefore $\varepsilon \in \mathcal{K}_{p}$.

Note that in the next lemma one does not assume that $S$ is unambiguous.

Lemma 5.5. Let $S$ be a compact semigroup, and let $(u, v) \in \mathfrak{F}(S)$. Let $e$ be an idempotent stabilizing $(u, v)$. If $f$ is an idempotent J-equivalent to $e$, then $f$ stabilizes an element of the $\sim$-class $p$ of $(u, v)$. Moreover, if e labels a loop of $\mathcal{K}_{p}$, then $f$ also labels a loop of $\mathcal{K}_{p}$.

Proof: If $f \mathcal{J} e$, then there are in the $\mathcal{J}$-class of $e$ some elements $s, t$ such that $s t s=s, t s t=t, s t=e, t s=f$. We have the four edges in $\mathcal{T}(S)$ which are depicted in Figure 1. In particular, $f$ stabilizes a vertex $\sim$-equivalent to $(u, v)$.

Denote by $\varepsilon, \sigma, \phi, \tau$ the edges in Figure 1 labeled by $e, s, f, t$, respectively. Since $s=e s, t=t e, f=t s$, we have $\sigma=\varepsilon \sigma, \tau=\tau \varepsilon$, and $\phi=\tau \sigma$. Therefore, if $\varepsilon$ belongs to the ideal $\mathcal{K}_{p}$, then all edges in Figure 1 belong to $\mathcal{K}_{p}$, and so $f$ labels a loop of $\mathcal{K}_{p}$.

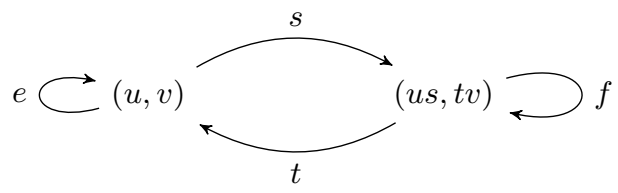

Figure 1. Edges in $\mathcal{T}(S)$.

Corollary 5.6. Let $S$ be a compact semigroup, and let $p \in \mathfrak{L}(S)$. Every idempotent of $J_{p}$ labels a loop of $\mathcal{K}_{p}$.

For $e \in J_{p}$, denote by $p_{e}$ the nonempty set of elements of $p$ stabilized by $e$. 
Proposition 5.7. Let $S$ be a compact unambiguous semigroup. Let $p \in$ $\mathfrak{L}(S)$. Then $J_{p}$ is the set of labels of edges of $\mathcal{K}_{p}$. Moreover, if $s \in J_{p}$ and $e$ and $f$ are idempotents such that $e \mathcal{R} s \mathcal{L} f$, then $s$ labels an edge from $p_{e}$ to $p_{f}$. Moreover, there is a bijection $p_{e} \rightarrow p_{f}$, given by $\mu_{s}(u, v)=$ $(u s, t v)$, where $t$ is the unique $t \in J_{p}$ such that $s t=e$ and $t s=f$.

Proof: Let $s$ be an element of $J_{p}$ and let $e$ and $f$ be idempotents such that $e \mathcal{R} s \mathcal{L} f$. Then there exists (a unique) $t \in J_{p}$ such that $s t=e$ and $t s=f$, for which we have $e \mathcal{L} t \mathcal{R} f$. Let $(u, v) \in p_{e}$. Note that such a pair $(u, v)$ exists by Corollary 5.6. Therefore, we are in the same situation as in the proof of Lemma 5.5, with the four edges depicted in Figure 1 belonging to $\mathcal{K}_{p}$ by Corollary 5.4. If there is another edge $(u, v) \stackrel{s}{\rightarrow}(x, y)$ in $\mathcal{K}_{p}$ with $(x, y) \in p_{f}$, then $x=u s$ and $v=s y$, thus $y=f y=t s y=t v$. Hence, there is for each vertex in $p_{e}$ exactly one edge labeled $s$ into a vertex of $p_{f}$. This defines the function $\mu_{s}: p_{e} \rightarrow p_{f}$ such that $\mu_{s}(u, v)=(u s, t v)$. Finally, note that $\mu_{s}$ and $\mu_{t}$ are mutually inverse.

We finish this section with a couple of observations concerning aperiodic semigroups, starting with the next lemma.

Lemma 5.8. Let $S$ be a compact aperiodic semigroup. Let $p \in \mathfrak{L}(S)$. If $(u, v),(x, y)$ are elements of $p$ stabilized by the same idempotent $e$ of $J_{p}$, then $(u, v)=(x, y)$.

Proof: Since $(u, v) \sim(x, y)$, there are $t, z \in S^{I}$ such that there are edges $(u, v) \stackrel{t}{\rightarrow}(x, y)$ and $(x, y) \stackrel{z}{\rightarrow}(u, v)$ in the category $\mathcal{T}(S)$. Then we also have edges as in Figure 2.

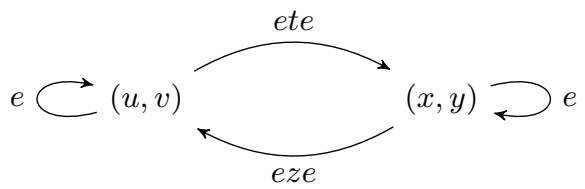

FIGURE 2 .

By Lemma 5.2 and stability of $S$, we conclude that ete and eze are $\mathcal{H}$-equivalent to $e$, thus, by aperiodicity, we get ete $=e z e=e$. By the definition of the category $\mathcal{T}(S)$, we deduce that $u=u e=x$ and $v=$ $e y=y$.

In the following result, we have a case in which the idempotents of $J_{p}$ parameterize the elements of $p$. 
Proposition 5.9. Let $S$ be a compact and unambiguous aperiodic semigroup. Let $p \in \mathfrak{L}(S)$. Then there is a bijection between the $\sim$-class $p$ and the set of idempotents in $J_{p}$, sending each $(u, v)$ to the unique idempotent $e \in J_{p}$ that stabilizes $(u, v)$.

Proof: Let $(u, v) \in p$. There are in $J_{p}$ idempotents that stabilize $(u, v)$, as $J_{p}$ contains the minimum ideal of the monoid of stabilizers of $(u, v)$. If $e, f$ are idempotents of $J_{p}$ stabilizing $(u, v)$, then $e=f$ by Lemma 5.3. Hence, we can consider the function $\varepsilon: p \rightarrow J_{p}$ sending $(u, v)$ to the unique idempotent of $J_{p}$ stabilizing $(u, v)$. The function $\varepsilon$ is injective by Lemma 5.8, and it is surjective by Corollary 5.6.

\section{Finitely cancelable semigroups}

Consider a compact semigroup $S$ generated by a closed set $A$. Recall that, in the context of topological semigroups, that means that every element of $S$ is arbitrarily close to products of elements of $A$. Note that, since $A$ is closed, we have $S=S^{I} A=A S^{I}$. Indeed, every element of $S$ is the limit of a net of the form $\left(w_{i} a_{i}\right)_{i \in I}$, where the $a_{i} \in A$ and the $w_{i}$ are perhaps empty products of elements of $A$. By compactness, we may assume that the nets $\left(w_{i}\right)_{i \in I}$ and $\left(a_{i}\right)_{i \in I}$ converge in $S^{I}$, say to $w$ and $a$, respectively. Since $A$ is closed, we conclude that $a \in A$, which shows that $S \subseteq S^{I} A$.

Say that $S$ is right finitely cancelable with respect to $A$ when, for every $a, b \in A$ and $u, v \in S^{I}$, the equality $u a=v b$ implies $a=b$ and $u=v$. This implies $A \cap S A=A \cap A S=\emptyset$.

Say that $S$ is right finitely cancelable if it is finitely cancelable with respect to some closed generating subset $A$. It turns out that the set $A$ is uniquely determined by $S$, as shown next.

Lemma 6.1. Let $S$ be a compact semigroup generated by closed subsets $A$ and $B$ such that $A \cap S A=B \cap S B=\emptyset$. Then we have $A=B$. In particular, if $S$ is right finitely cancelable with respect to both $A$ and $B$, then $A=B$.

Proof: Let $a \in A$. Since $S=S^{I} B=S^{I} A$, we have $a=s b$ for some $s \in$ $S^{I}$ and $b \in B$, and $b=t c$ for some $t \in S^{I}$ and $c \in A$. We obtain the factorization $a=s t c$. Since $A \cap S A=\emptyset$, we must have $s=t=1$, and so $a=b \in B$, showing that $A \subseteq B$. By symmetry, we have $B \subseteq A$.

Say that a pseudovariety of semigroups is right finitely cancelable if $\bar{\Omega}_{A} \mathrm{~V}$ is right finitely cancelable with respect to $A$, for every finite alphabet $A$. 
Proposition 6.2. A pseudovariety of semigroups $\mathrm{V}$ is right finitely cancelable if and only if $\mathrm{V}=\mathrm{D} * \mathrm{~V}$.

Proof: It is observed in [7] that $\mathrm{V}$ is right finitely cancelable if and only if, for every finite alphabet $A$, and for every $\vee$-recognizable language $L$ of $A^{+}$and $a \in A$, the language $L a$ is also $\mathrm{V}$-recognizable. In [37] one finds a proof that this is equivalent to $\mathrm{V}=\mathrm{D} * \mathrm{~V}$.

The above definitions have obvious duals which are obtained by replacing right by left. Note that a semigroup pseudovariety $\mathrm{V}$ is right finitely cancelable if and only the pseudovariety $\mathrm{V}^{\mathrm{op}}$ of semigroups of $\mathrm{V}$ with reversed multiplications is left finitely cancelable. We say that a compact semigroup is finitely cancelable (with respect to $A$ ) if it is simultaneously right and left finitely cancelable (with respect to $A$ ). Similarly, a pseudovariety of semigroups is finitely cancelable if it is simultaneously right and left finitely cancelable.

Example 6.3. If $\mathrm{V}$ is a semigroup pseudovariety containing some nontrivial monoid and such that $\mathrm{V}=\mathrm{V} * \mathrm{D}$, then $\mathrm{V}$ is finitely cancelable (cf. [1, Exercise 10.2.10] and [16, Proposition 1.60]).

The following proposition is [6, Proposition 6.3].

Proposition 6.4. If $\mathrm{V}$ is an equidivisible pseudovariety of semigroups not contained in $\mathrm{CS}$, then $\mathrm{V}$ is finitely cancelable.

The next lemma is the first of a series of results in which the hypothesis of a semigroup being finitely cancelable enables us to get further insight into the quasi-order of 2-factorizations.

Lemma 6.5. Suppose $S$ is a compact semigroup, finitely cancelable with respect to $A$. Let $u, v \in S^{I}$ and $a \in A$. If the $\sim$-class of at least one of $(u a, v)$ and $(u, a v)$ is not a singleton, then $(u, a v) \sim(u a, v)$.

Proof: By duality, it suffices to consider the case where the $\sim$-class of $p=$ $(u a, v)$ is not a singleton. Let $q$ be in $p / \sim$ with $p \neq q$. As $p \leq q$, we may consider a transition $x$ from $p$ to $q$. Then we have $q=($ uax, $y)$ for some $y \in S^{I}$ such that $v=x y$. Since $q \leq p$, there is $t$ such that $u a=$ uaxt and $y=t x y$. Because $p \neq q$, we must have $t \neq 1$, whence we may take $b \in A$ and $z \in S^{I}$ such that $t=z b$. Because $S$ is finitely cancelable with respect to $A$, from $u a=u a x t=u a x z b$ we get $a=b$ and $u=(u a)(x z)$. On the other hand, we have $(x z)(a v)=x(z a) v=x t x y=x y=v$, which shows that $(u a, v) \sim(u, a v)$.

We now turn our attention to profinite semigroups. 
Proposition 6.6. Suppose $S$ is a profinite semigroup generated by a closed subset $A$. Let $p, q \in \mathfrak{F}(s)$ with $p<q$. Then, there are $x, y \in S^{I}$ and $a \in A$ such that

$$
p \leq(x, a y)<(x a, y) \leq q .
$$

Proof: Let $p=(u, v)$ and $q=\left(u^{\prime}, v^{\prime}\right)$. Since $(u, v)<\left(u^{\prime}, v^{\prime}\right)$, there exists $t \in S$ such that $u^{\prime}=u t$ and $v=t v^{\prime}$, and the system

$$
\left\{\begin{array}{l}
u t X=u, \\
X t v^{\prime}=v^{\prime}
\end{array}\right.
$$

has no solution $X \in S$. By a standard compactness argument which can be found in the proof of [1, Theorem 5.6.1], there is some continuous onto homomorphism $\varphi_{0}: S \rightarrow R$, with $R$ finite, which may be naturally extended to an onto continuous homomorphism $\varphi: S^{I} \rightarrow R^{I}$, and such that the following system (6.2) has no solution $X \in R$ :

$$
\left\{\begin{array}{l}
\varphi(u) \varphi(t) X=\varphi(u), \\
X \varphi(t) \varphi\left(v^{\prime}\right)=\varphi\left(v^{\prime}\right) .
\end{array}\right.
$$

Let $\left(t_{n}\right)_{n}$ be a net of elements of the (discrete) subsemigroup of $S$ generated by $A$ such that $\left(t_{n}\right)_{n}$ converges to $t$ and such that $\varphi\left(t_{n}\right)=\varphi(t)$ for all $n$. Write $t_{n}=a_{n, 0} a_{n, 1} \cdots a_{n, k_{n}}$, with the $a_{n, i} \in A$. Then the following inequalities hold for $i=0, \ldots, k_{n}$ :

$$
\begin{aligned}
\left(\varphi\left(u a_{n, 0} \cdots a_{n, i-1}\right), \varphi\right. & \left.\left(a_{n, i} \cdots a_{n, k_{n}} v^{\prime}\right)\right) \\
\leq & \left(\varphi\left(u a_{n, 0} \cdots a_{n, i}\right), \varphi\left(a_{n, i+1} \cdots a_{n, k_{n}} v^{\prime}\right)\right) .
\end{aligned}
$$

Since $\leq$ is a transitive relation and the non-existence of a solution to (6.2) guarantees that the following strict inequality holds

$$
\left(\varphi(u), \varphi\left(a_{n, 0} \cdots a_{n, k_{n}} v^{\prime}\right)\right)<\left(\varphi\left(u a_{n, 0} \cdots a_{n, k_{n}}\right), \varphi\left(v^{\prime}\right)\right),
$$

we deduce that there is $i=i_{n}$ such that the inequality (6.3) is also strict. As $A$ is closed and $S$ is compact, by taking subnets we may assume that the net $\left(a_{n, i_{n}}\right)_{n}$ converges to some $a \in A$, that $\varphi\left(a_{n, i_{n}}\right)=\varphi(a)$ for every $n$, and that each of the nets $t_{n}^{\prime}=a_{n, 0} \cdots a_{n, i_{n}-1}$ and $t_{n}^{\prime \prime}=$ $a_{n, i_{n}+1} \cdots a_{n, k_{n}}$ converges to some $t^{\prime}, t^{\prime \prime} \in S^{I}$, respectively (in particular, this yields $\left.t=t^{\prime} a t^{\prime \prime}\right)$. Then the strict inequality in (6.3), with $i=i_{n}$, yields $\left(\varphi\left(u t^{\prime}\right), \varphi\left(a t^{\prime \prime} v^{\prime}\right)\right)<\left(\varphi\left(u t^{\prime} a\right), \varphi\left(t^{\prime \prime} v^{\prime}\right)\right)$, which implies that

$$
p=\left(u, t^{\prime} a t^{\prime \prime} v^{\prime}\right) \leq\left(u t^{\prime}, a t^{\prime \prime} v^{\prime}\right)<\left(u t^{\prime} a, t^{\prime \prime} v^{\prime}\right) \leq\left(u t^{\prime} a t^{\prime \prime}, v^{\prime}\right)=q .
$$

Thus, it suffices to choose $x=u t^{\prime}$ and $y=t^{\prime \prime} v^{\prime}$ to obtain the inequalities of the statement of the proposition. 
We close this subsection with a result regarding the existence of a successor in the quasi-ordered set of 2-factorizations.

Proposition 6.7. Suppose $S$ is a profinite semigroup, finitely cancelable with respect to $A$. Let $p, q \in \mathfrak{F}(s)$ and suppose that $p<q$.

(a) Consider the unique $u, v$, a such that $u, v \in S^{I}, a \in A$, and $p=(u, a v)$. If $p \prec q$, then we have

$$
p=(u, a v) \prec(u a, v)=q .
$$

Moreover, the $\sim$-classes of $p$ and $q$ are singletons.

(b) Conversely, if $p=(u, a v)$ and $q=(u a, v)$, where $u, v \in S^{I}$ and $a \in A$, then we have $p \prec q$.

Proof: (a) Notice that $u, v, a$ really exist and are unique. Indeed, take $p=(u, w)$, with $u, w \in S^{I}$. One has $w \neq 1$, because $p<q$, and so $w=a v$ for some $a \in A$ and $v \in S^{I}$, which are unique because $S$ is finitely cancelable with respect to $A$. By Proposition 6.6, there are $u^{\prime}, v^{\prime} \in S^{I}$ and $a^{\prime} \in A$ such that $p \leq\left(u^{\prime}, a^{\prime} v^{\prime}\right)<\left(u^{\prime} a^{\prime}, v^{\prime}\right) \leq q$. Since $p \prec q$, we must have $p \sim\left(u^{\prime}, a^{\prime} v^{\prime}\right)<\left(u^{\prime} a^{\prime}, v^{\prime}\right) \sim q$. It then follows from Lemma 6.5 that the $\sim$-classes of $\left(u^{\prime}, a^{\prime} v^{\prime}\right)$ and $\left(u^{\prime} a^{\prime}, v^{\prime}\right)$ are singletons, thus $p=\left(u^{\prime}, a^{\prime} v^{\prime}\right)$ and $q=\left(u^{\prime} a^{\prime}, v^{\prime}\right)$. By the uniqueness of $u$, $v$, and $a$, we then have $q=(u a, v)$

(b) Assume there exists $r=(x, y)$ with $p<r<q$. There are $z, t$ such that $x=u t, a v=t y, u a=x z$, and $y=z v$. If $t=1$, then $r=p$, while if $z=1$, then $r=q$, hence both $t$ and $z$ are different from 1 . Since $a v=t y$, from the fact that $S$ is finitely cancelable with respect to $A$, it follows that there is $t^{\prime}$ such that $t=a t^{\prime}$ and $v=t^{\prime} y$. Similarly, there is $z^{\prime}$ such that $z=z^{\prime} a$ and $u=x z^{\prime}$. Therefore, $u=x z^{\prime}=u t z^{\prime}=u a \cdot t^{\prime} z^{\prime}$ and $v=t^{\prime} y=t^{\prime} z v=t^{\prime} z^{\prime} \cdot a v$. This shows that $p \sim q$, in contradiction with $p<q$. Hence $p \prec q$.

\section{Step points and stationary points}

In this section, we continue gathering important properties of the linear orders induced by pseudowords. We identify two types of elements in such orders, that we call step points and stationary points. Let us start by introducing these notions.

Let $L$ be a partially ordered set. We call step points the points of $L$ that admit either a successor or a predecessor, or are the minimum or the maximum of $L$, if they exist. All other points are said to be stationary. The set of step points of $L$ will be denoted by $\operatorname{step}(L)$, and the set of stationary points of $L$ will be denoted by $\operatorname{stat}(L)$. 
For an element $s$ of a semigroup $S$, we also say that $p \in \mathfrak{F}(s)$ is a step point (respectively, a stationary point) if $\chi(p)$ is a step point (respectively, a stationary point) of $\mathfrak{L}(s)$. In this section we further develop the results obtained in Section 6 , using the notions of step point and stationary point. If $S$ is profinite and finitely cancelable, then the $\sim$-class $p$ of a step point $(u, v)$ of $\mathfrak{F}(S)$ is a singleton (cf. Proposition $6.7(\mathrm{a})$ ), for which reason, in that case, we feel free to make the abuse of notation $p=(u, v)$.

As preparation for the following example, recall that in a compact semigroup $S$, if $s \in S$, then $s^{\omega}$ denotes the unique idempotent in the closed subsemigroup of $S$ generated by $s$. Later on, we shall also make use of the notation $s^{\omega+1}$ for $s^{\omega} s$, and $s^{\omega-1}$ for the inverse of $s^{\omega+1}$ in the maximal subgroup containing $s^{\omega+1}$.

Example 7.1. Consider the pseudoword $a^{\omega}$ of the free pro-aperiodic semigroup $\bar{\Omega}_{\{a\}}$ A. Then $\mathfrak{F}\left(a^{\omega}\right)$ has only one stationary point, namely $\left(a^{\omega}, a^{\omega}\right)$. The set $\mathfrak{F}\left(a^{\omega}\right)$ is linearly ordered, whence isomorphic to $\mathfrak{L}\left(a^{\omega}\right)$, with order type $\omega+1+\omega^{*}$. More precisely, its elements are ordered as follows:

$$
\begin{aligned}
\left(1, a^{\omega}\right)<\left(a, a^{\omega}\right)<\left(a^{2}, a^{\omega}\right)<\cdots< & \left(a^{\omega}, a^{\omega}\right)<\cdots \\
& \cdots<\left(a^{\omega}, a^{2}\right)<\left(a^{\omega}, a\right)<\left(a^{\omega}, 1\right) .
\end{aligned}
$$

Example 7.1 should be compared with the following one.

Example 7.2. Consider the pseudoword $a^{\omega}$ of the free profinite semigroup $\bar{\Omega}_{A}$ S. Like in Example 7.1, $\mathfrak{L}\left(a^{\omega}\right)$ has order type $\omega+1+\omega^{*}$, and its sole stationary point is $p=\left(a^{\omega}, a^{\omega}\right) / \sim$ :

$$
\begin{gathered}
\left(1, a^{\omega}\right)<\left(a, a^{\omega-1}\right)<\left(a^{2}, a^{\omega-2}\right)<\cdots<p<\cdots \\
\cdots<\left(a^{\omega-2}, a^{2}\right)<\left(a^{\omega-1}, a\right)<\left(a^{\omega}, 1\right) .
\end{gathered}
$$

But $\left(a^{\omega}, a^{\omega}\right) / \sim$ has infinitely many elements, namely, the pairs of the form $\left(g, g^{\omega-1}\right)$, where $g$ is an element in the maximal subgroup contain$\operatorname{ing} a^{\omega}$.

Examples 7.1 and 7.2 fit in the following definition.

Definition 7.3 (Clustered sets). We say that the partially ordered set $L$ is clustered if the following conditions hold:

(C.1) $L$ has a minimum min $L$ and a maximum $\max L$;

(C.2) for every $q \in L$, if $q=\min L$ or $q$ has a predecessor, then $q$ has a successor or $q=\max L$;

(C.3) for every $q \in L$, if $q=\max L$ or $q$ has a successor, then $q$ has a predecessor or $q=\min L$;

(C.4) for every $p, q \in L$, if $] p, q[$ is nonempty, then there is a step point in the interval $] p, q[$. 
Property (C.4) translates into saying that the set of step points of $L$ is dense with respect to the order topology of $L$.

Theorem 7.4. Let $S$ be a profinite semigroup which is finitely cancelable, and let $s \in S$. Then $\mathfrak{L}(s)$ is clustered.

Proof: Property (C.1) in Definition 7.3 holds trivially, with $\min \mathfrak{L}(s)=$ $(1, s)$ and $\max \mathfrak{L}(s)=(s, 1)$.

Let us show (C.2). Take $s \in S$ and $p, q \in \mathfrak{F}(s)$ with $p \prec q$. We have to show that either $q$ has a successor, or $q=\max \mathfrak{L}(s)$. Let $A$ be the generating set with respect to which $S$ is finitely cancelable. By Proposition 6.7, there are $u, v \in S^{I}$ and $a \in A$ with $p=(u, a v)$ and $q=(u a, v)$. If $v=1$, then $q=\max \mathfrak{L}(s)$, so that we may assume $v \neq 1$. Let $v=b w$ with $b \in A$, and let $r=(u a b, w)$. Clearly, we have $q \leq r$. We claim that $q \prec r$. Indeed, if $q=r$, then $u a=u a b$ and $b w=w$. Therefore, $a=b, u=u a$, and $a v=a a w=a w=v$, showing that $p=q$, in contradiction with the hypothesis. Hence, we must have $q<r$, since otherwise we would obtain $q \sim r$ and $q \neq r$, which entails $p \sim q$ by Lemma 6.5. Finally, by Proposition 6.7(b) applied to $q$ and $r$, we get $q \prec r$. This establishes (C.2), and (C.3) holds dually.

Finally, let us prove (C.4). If $p$ is a step point, then $] p, q[\neq \emptyset$ implies that the successor of $p$ belongs to $] p, q[$. Hence, it suffices to consider the case where $p$ is stationary. Let $\hat{p} \in \chi^{-1}(p)$ and $\hat{q} \in \chi^{-1}(q)$. By Proposition 6.6, there are $u, v \in S^{I}$ and $a \in A$ such that $\hat{p} \leq(u, a v)<$ $(u a, v) \leq \hat{q}$ in $\mathfrak{F}(s)$. By Proposition 6.7(b), we have $(u, a v) \prec(u a, v)$. Therefore, $(u, a v)$ and $(u a, v)$ are step points. In particular, we have $(u, a v) \in] p, q[\cap \operatorname{step}(\mathfrak{L}(s))$.

In the next result, we characterize the stationary points as the vertices with a nontrivial local monoid in the category of transitions.

Proposition 7.5. Let $S$ be a profinite semigroup which is finitely cancelable. Let $(u, v) \in \mathfrak{F}(S)$. Then $(u, v)$ is a stationary point if and only if it is stabilized by some element of $S$.

Proof: Suppose that $(u, v)$ is stationary. Let $A$ be the set with respect to which $S$ is finitely cancelable. Since $v \neq 1$, we may take a factorization $v=a w$ with $a \in A$ and $w \in S^{I}$. Clearly, $(u, a w) \leq(u a, w)$ holds, and so $(u, a w) \sim(u a, w)$ by Proposition 6.7(b). Hence, there is an edge $(u a, w) \stackrel{t}{\rightarrow}(u, a w)$, for some $t \in S^{I}$. This implies that at $\in S$ stabilizes $(u, v)$. 
Conversely, suppose there is $z \in S$ stabilizing $(u, v)$. There is some factorization of the form $z=a t$, for some $a \in A$ and $t \in S^{I}$. The following equalities and inequalities

$$
(u, v)=(u, a t v) \leq(u a, t v) \leq(u a t, v)=(u, v)
$$

show that $(u, v)=(u, a t v) \sim(u a, t v)$. It follows from Proposition 6.7(a) that $(u, v)$ has no successor, since otherwise this successor would be $(u a, t v)$, in contradiction with $(u a, t v) \leq(u, v)$. Since $(u, v)$ is not the maximum of $\mathfrak{F}(s)$ (because $v=z v \neq 1$ ), it must be a stationary point.

Proposition 7.5 is applied in the proof of the next result.

Proposition 7.6. Let $S$ be an equidivisible profinite semigroup $S$ which is finitely cancelable. Then, in $\mathcal{T}(S)$, any two coterminal edges between elements of distinct strongly connected components are equal. In other words, if $(\alpha, \beta) \stackrel{t}{\rightarrow}(\gamma, \delta)$ and $(\alpha, \beta) \stackrel{s}{\rightarrow}(\gamma, \delta)$ are edges of $\mathcal{T}(S)$ such that $(\alpha, \beta)<(\gamma, \delta)$, then $t=s$.

Proof: The hypothesis translates into the following equalities: $\gamma=\alpha t=$ $\alpha s$ and $\beta=t \delta=s \delta$. We first assume that at least one of the points $(\alpha, \beta)$ and $(\gamma, \delta)$ is a step point. By symmetry, we may as well assume that $(\alpha, \beta)$ is a step point. From the equality $\alpha t=\alpha s$, by equidivisibility and without loss of generality, we may assume that there is some $z \in S^{I}$ such that $\alpha z=\alpha$ and $t=z s$. Then, we have $z \beta=z s \delta=t \delta=\beta$. This shows that $(\alpha, \beta) \stackrel{z}{\rightarrow}(\alpha, \beta)$ is a loop of $\mathcal{T}(w)$. Since $(\alpha, \beta)$ is assumed to be a step point, applying Proposition 7.5 we obtain $z=1$, thus $t=s$.

It remains to consider the case where both $(\alpha, \beta)$ and $(\gamma, \delta)$ are stationary. By Theorem 7.4 , there is a step point $(x, y) \in \mathfrak{F}(w)$ such that $(\alpha, \beta)<(x, y)<(\gamma, \delta)$. By the preceding paragraph, there are unique edges in $\mathcal{T}(w)$ from $(\alpha, \beta)$ to $(x, y)$ and from $(x, y)$ to $(\gamma, \delta)$. Let $r_{1}$ and $r_{2}$ be the respective labels. To prove the proposition, it suffices to show that $\tau=r_{1} r_{2}$ whenever $(\alpha, \beta) \stackrel{\tau}{\rightarrow}(\gamma, \delta)$ is an edge of $\mathcal{T}(w)$.

Note that $\left(x, r_{2}\right)$ and $(\alpha, \tau)$ are elements of $\mathfrak{F}(\gamma)$. Since $\beta=\tau \delta$ and $y=r_{2} \delta$, if $\left(x, r_{2}\right) \leq(\alpha, \tau)($ in $\mathfrak{F}(\gamma))$, then $(x, y) \leq(\alpha, \beta)($ in $\mathfrak{F}(w))$ by Remark 4.6 , which gives a contradiction. Hence, by equidivisibility, there is in $\mathcal{T}(\gamma)$ an edge $(\alpha, \tau) \stackrel{r_{0}}{\longrightarrow}\left(x, r_{2}\right)$. In particular, we have $\tau=r_{0} r_{2}$. Remark 4.6 guarantees the existence of the edge $(\alpha, \beta) \stackrel{r_{0}}{\longrightarrow}(x, y)$. But we defined, in the previous paragraph, the edge $(\alpha, \beta) \stackrel{r_{1}}{\longrightarrow}(x, y)$ as the unique edge from $(\alpha, \beta)$ to $(x, y)$. Therefore $r_{0}=r_{1}$, and so we have the equality $\tau=r_{1} r_{2}$, which we have seen to be sufficient to conclude the proof. 
Corollary 7.7. Let $S$ be an equidivisible profinite semigroup $S$ which is finitely cancelable. Let $p_{1}, p_{2} \in \mathfrak{L}(w)$. If $p_{1}<p_{2}$ then the set of transitions from $p_{1}$ to $p_{2}$ is contained in a J-class of $S$.

Proof: Let $x_{1}, x_{1}^{\prime} \in p_{1}$ and $x_{2}, x_{2}^{\prime} \in p_{2}$, and consider edges $x_{1} \stackrel{t}{\rightarrow} x_{2}$ and $x_{1}^{\prime} \stackrel{t^{\prime}}{\rightarrow} x_{2}^{\prime}$. Then we have edges $x_{i} \stackrel{s_{i}}{\longrightarrow} x_{i}^{\prime}$ and $x_{i}^{\prime} \stackrel{r_{i}}{\longrightarrow} x_{i}$, and also $x_{1} \stackrel{s_{1} t^{\prime} r_{2}}{\longrightarrow} x_{2}$ and $x_{1}^{\prime} \stackrel{r_{1} t s_{2}}{\longrightarrow} x_{2}^{\prime}$. By Proposition 7.6, we must have $t=$ $s_{1} t^{\prime} r_{2}$ and $t^{\prime}=r_{1} t s_{2}$, whence $t \mathcal{J} t^{\prime}$.

Remark 7.8. We remark that there is a large class of pseudovarieties whose corresponding finitely generated relatively free profinite semigroups satisfy the hypotheses of Proposition 7.6. Let $\mathrm{V}$ be a pseudovariety of semigroups such that $\mathrm{V}=\mathrm{LI} \oplus \mathrm{V}$. Then every semigroup of the form $\bar{\Omega}_{A} \mathrm{~V}$, with $A$ a finite alphabet, satisfies all conditions in Proposition 7.6: they are profinite, equidivisible, and finitely cancelable (cf. Theorem 3.3 and Proposition 6.4, where the latter may be applied because $\mathrm{V}=\mathrm{LI}(\mathrm{m} \mathrm{V}$ implies $\mathrm{V} \supseteq \mathrm{LI}$ and thus $\mathrm{V} \not \subset \mathrm{CS})$.

The pseudovarieties of the form $\mathrm{V}=\mathrm{LI} / \mathrm{O}$ are closed under the twosided Karnofsky-Rhodes expansion, and therefore also under the corresponding profinite expansion that is defined according to the general method given in [39]. From this it easily follows that Proposition 7.6 holds in the case where $S$ is a semigroup of the form $\bar{\Omega}_{A} \mathrm{~V}$ for some finite alphabet $A$ and some pseudovariety $\mathrm{V}$ such that $\mathrm{V}=\mathrm{LI} \oplus \mathrm{V}$. Indeed, in that case, the (profinite) two-sided Karnofsky-Rhodes expansion of $\bar{\Omega}_{A} \mathrm{~V}$ with respect to the generating mapping $\bar{\Omega}_{A} \mathrm{~V} \rightarrow \bar{\Omega}_{A} \mathrm{~V}$ is isomorphic to $\bar{\Omega}_{A} \mathrm{~V}$, which straightforwardly entails that $\mathcal{T}\left(\bar{\Omega}_{A} \mathrm{~V}\right)$ does not have distinct edges between two connected components.

Proposition 7.6 is used in the proof of the following lemma, establishing a sufficient condition for equality between stationary points.

Lemma 7.9. Let $S$ be an equidivisible profinite semigroup which is finitely cancelable. Let $p$ and $q$ be stationary points of $\mathfrak{L}(S)$. If there is a transition $p \stackrel{t}{\rightarrow} q$ such that $t$ lies $\mathcal{J}$-above both $J_{p}$ and $J_{q}$ then $p=q$.

Proof: We have $p \leq q$, so, arguing by contradiction, suppose that $p<q$. Consider an edge $(u, v) \stackrel{t}{\rightarrow}(x, y)$ of $\mathcal{T}(S)$ with $(u, v) \in p$ and $(x, y) \in q$. Let $e \in J_{p}$ and $f \in J_{q}$ be idempotents respectively stabilizing $(u, v)$ and $(x, y)$. Then we have a transition $(u, v) \stackrel{\text { etf }}{\longrightarrow}(x, y)$. By Proposition 7.6, it follows that et $f=t$, whence, since $S$ is stable, and by the hypothesis that $t$ lies $\mathcal{J}$-above both $e$ and $f$, we have $e \mathcal{R} t \mathcal{L} f$. Therefore, there exists $s \in J_{p}=J_{q}$ such that $t s=e$ and $s t=f$. Now, the 
assumption that we have a transition $(u, v) \stackrel{t}{\rightarrow}(x, y)$ means that the equalities $u t=x$ and $v=t y$ hold. Combining with the equalities $u e=u$ and $f y=y$, we deduce that $x s=u t s=u e=u$ and $s v=s t y=f y=y$. Hence $(x, y) \leq(u, v)$, which contradicts the assumption $p<q$. To avoid the contradiction, we must have $p=q$.

The following is an example obtained by application of Lemma 7.9.

Example 7.10. Let $\mathrm{V}$ be a pseudovariety containing LSI, and let $A$ be a finite alphabet. It is shown in [2] (see also [4]), using Zorn's Lemma and a standard compactness argument, that $\bar{\Omega}_{A} \mathrm{~V}$ contains regular elements that are $\mathcal{J}$-maximal among the elements of $\bar{\Omega}_{A} \vee \backslash A^{+}$. We next verify that if $\mathrm{V}$ is closed under concatenation and $u$ is a $\mathcal{J}$-maximal regular element of $\bar{\Omega}_{A} \mathrm{~V}$, then the order type of $\mathfrak{L}(u)$ is $\omega+1+\omega^{*}$. Indeed, let $p, q$ be two stationary points of $\mathfrak{L}(u)$ such that $p \leq q$, and let $e \in J_{p}$, $f \in J_{q}$. From the maximality assumption on $u$, we deduce that $e \mathcal{J} u \mathcal{J} f$. Hence $p=q$ by Lemma 7.9 , showing that $\mathfrak{L}(u)$ has only one stationary point.

Restricting our attention to the case of profinite semigroups that are free relatively to pseudovarieties closed under concatenation, we obtain stronger results about step and stationary points. The next lemma is a provisional result with this flavor, which is improved later on, namely in Proposition 7.13 .

Lemma 7.11. Let $A$ be a finite alphabet, and let $\mathrm{V}$ be a pseudovariety closed under concatenation. Let $w \in \bar{\Omega}_{A} \mathrm{~V}$. If $(u, v)$ is a stationary point of $\mathfrak{F}(w)$, then there exists a strictly increasing sequence $\left(u_{n}, v_{n}\right)_{n}$ of points of $\mathfrak{F}(w)$ such that $\lim u_{n}=u$ and $\lim v_{n}=v$.

Proof: Since $A$ is finite, $\bar{\Omega}_{A} \vee$ is metrizable, and so $\mathfrak{L}(w)$ is metrizable by Corollary 4.4. Let $p=(u, v) / \sim$. By Theorem 7.4, there is a strictly increasing sequence $\left(x_{n}, y_{n}\right)_{n}$ of step points converging in $\mathfrak{L}(w)$ to $p$. As $S$ is compact, taking subsequences we may assume that $\left(x_{n}, y_{n}\right)_{n}$ converges in $\mathfrak{F}(w)$ to some $(x, y) \in \mathfrak{F}(w)$. By Proposition 4.3, we know that $(x, y) \in$ $p$. Then there are edges $(x, y) \stackrel{s}{\rightarrow}(u, v)$ and $(u, v) \stackrel{t}{\rightarrow}(x, y)$ in $\mathcal{T}(w)$. Note that $\lim x_{n}=x=x$ st. Hence, by Theorem 3.1 and Lemma 3.2, for every $n$ there is a factorization $x_{n}=x_{n}^{\prime} s_{n} t_{n}$ with $\lim x_{n}^{\prime}=x$, $\lim s_{n}=s$, and $\lim t_{n}=t$. Then, we have $\lim \left(x_{n}^{\prime} s_{n}, t_{n} y_{n}\right)=(u, v)$ in $\mathfrak{F}(w)$, thus $\lim \left(x_{n}^{\prime} s_{n}, t_{n} y_{n}\right) / \sim=p$ in $\mathfrak{L}(w)$ by Proposition 4.3. And since $\left(x_{n}^{\prime} s_{n}, t_{n} y_{n}\right) \leq\left(x_{n}, y_{n}\right)<(x, y)$, we conclude that the sequence $\left(\left(x_{n}^{\prime} s_{n}, t_{n} y_{n}\right) / \sim\right)_{n}$ has $p$ as supremum but not as maximum, enabling us to extract from the sequence $\left(x_{n}^{\prime} s_{n}, t_{n} y_{n}\right)_{n}$ a strictly increasing subsequence. Since this subsequence also converges to $(u, v)$ in $\mathfrak{F}(w)$, this completes the proof. 
Lemma 7.12. Let $A$ be a finite alphabet, and let $\mathrm{V}$ be a pseudovariety closed under concatenation. Let $w \in \bar{\Omega}_{A} \vee$ and let $K$ be a nonempty clopen subset of $\mathfrak{F}(w)$. Then $K$ has minimum and maximum, and both of them are step points.

Proof: As $K$ is closed, whence compact, we know from Proposition 4.3 that its projection under $\chi$ is also compact, whence closed. Let $p=$ $\inf \chi(K)$ and $q=\sup \chi(K)$. Since $\chi(K)$ is closed, we have $p=\min \chi(K)$ and $q=\max \chi(K)$. Hence, there exist $\hat{p} \in K \cap \chi^{-1}(p)$ and $\hat{q} \in K \cap$ $\chi^{-1}(q)$. Although these points are perhaps not unique, all choices are $\sim$-equivalent. Moreover, for every other point $r \in K$, from $\chi(r) \in[p, q]$ we deduce that $\hat{p} \leq r \leq \hat{q}$.

It remains to show that $\hat{p}$ and $\hat{q}$ are step points. Suppose on the contrary that $\hat{p}$ is a stationary point. By Lemma 7.11 , there is a strictly increasing sequence $\left(\hat{p}_{n}\right)_{n}$ in $\mathfrak{F}(w)$ converging to $\hat{p}$. Since $K$ is open, it must contain points of the form $\hat{p}_{n}$. As $\hat{p}_{n}<\hat{p}$, this contradicts the fact that $\hat{p}$ is the minimum of $K$. Hence, $\hat{p}$ is a step point and, similarly, so is $\hat{q}$.

Lemma 7.11 was used to prove Lemma 7.12. We next use Lemma 7.12 to show that the sequence in Lemma 7.11 may be formed only by step points.

Proposition 7.13. Let $A$ be a finite alphabet, and let $\mathrm{V}$ be a pseudovariety closed under concatenation. Let $w \in \bar{\Omega}_{A} \mathrm{~V}$. If $(u, v)$ is a stationary point of $\mathfrak{F}(w)$, then there exists a strictly increasing sequence $\left(u_{n}, v_{n}\right)_{n}$ of step points of $\mathfrak{F}(w)$ such that $\lim u_{n}=u$ and $\lim v_{n}=v$.

Proof: Consider in $\mathfrak{F}(w)$ the ball $B\left((u, v), \frac{1}{n}\right)$ of radius $\frac{1}{n}$ centered at $(u, v)$. It is a clopen subset of $\mathfrak{F}(w)$. By Lemma 7.12, its minimum is a step point $\left(u_{n}, v_{n}\right)$. Clearly, the sequence $\left(u_{n}, v_{n}\right)_{n}$ contains some subsequence with the required properties.

\section{Cluster words}

In this section, we use the knowledge about the labeled linear orders induced by pseudowords over $A$ to obtain a representation theorem: every pseudoword over A may be represented by a (partially) labeled linear order having specific properties, which we introduce now.

By a partially labeled ordered set, we mean a pair $(P, f)$ such that $P$ is an ordered set and $f$ is a function (the labeling) with domain contained in $P$. An isomorphism between partially labeled ordered sets $(P, f)$ and $(Q, g)$ is an isomorphism $\varphi: P \rightarrow Q$ of ordered sets such that $\operatorname{Dom} g=\varphi(\operatorname{Dom} f)$ and $f(p)=g \circ \varphi(p)$ for every $p \in \operatorname{Dom} f$. 
Consider a profinite equidivisible semigroup $S$ which is finitely cancelable with respect to $A$. We may then consider the mappings i and t from $S^{I}$ to $A \uplus\{1\}$ such that $\mathrm{i}(1)=\mathrm{t}(1)=1$ and, for $s \in S$, the images $\mathrm{i}(s)$ and $\mathrm{t}(s)$ are respectively the unique prefix and the unique suffix of $s$ in $A$. For $s \in S$, denote by $\mathfrak{L}_{c}(s)$ the partially labeled linearly ordered set $(\mathfrak{L}(s), \ell)$ defined by the mapping $\ell$ : $\operatorname{step}(L) \rightarrow A \uplus\{1\}$ such that $\ell(u, v)=\mathrm{i}(v)$, for $(u, v) \in \operatorname{step}(\mathfrak{L}(s))$. Note that $\ell(p)=1$ if and only if $p=(s, 1)$. Recall from Theorem 7.4 that $\mathfrak{L}(s)$ is clustered. By a cluster word over $A$ we mean a partially labeled linearly ordered set $(L, \ell)$ such that $L$ is clustered, $\ell$ is a function $\operatorname{step}(L) \rightarrow A \uplus\{1\}$, and $\ell(p)=1$ if and only if $p=\max L$.

Example 8.1. For the pseudoword $w=\left(a^{\omega} b\right)^{\omega}$ of $\bar{\Omega}_{\{a, b\}} \mathrm{A}$, the cluster word $\mathfrak{L}_{c}(w)$ is described in Diagram (8.1),

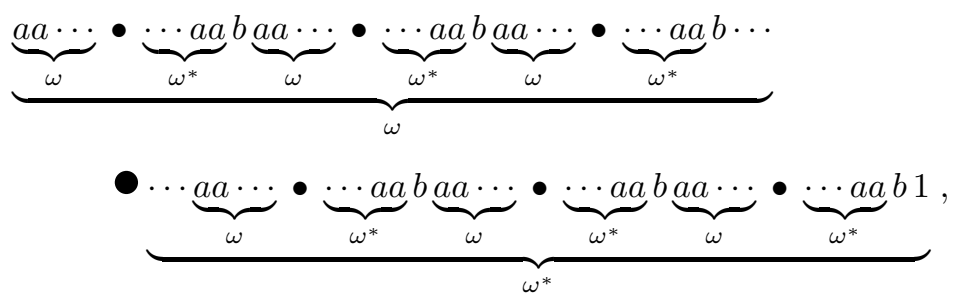

where each of the small bullets • represent a stationary point $q$ such that $a^{\omega} \in J_{q}$ and the bigger bullet represents the unique stationary point $r$ such that $\left(a^{\omega} b a^{\omega}\right)^{\omega} \in J_{r}$. Note that, in particular, the order type of $\mathfrak{L}(w)$ is $\left(\omega+1+\omega^{*}\right) \omega+1+\left(\omega+1+\omega^{*}\right) \omega^{*}$.

We next introduce, in Definition 8.2, a notion of algebraic recognition of cluster words inspired by the definition of automata recognizing words indexed by linear orders, introduced in [13]. A notable similarity resides in the role played by cofinal sets, whose definition we next recall. In a linearly ordered set $L$, a subset $X$ of $L$ is left cofinal at $p$ if $X \cap] q, p[\neq \emptyset$ for every $q<p$, right cofinal at $p$ if $X \cap] p, q[\neq \emptyset$ for every $q>p$, and cofinal if it is right or left cofinal at $p$. Cofinality of sets may be viewed as a sort of Büchi recognizability condition [35]. In other words, it captures in finite recognizing devices the behavior at limit points.

Definition 8.2. Let $\varphi: A \rightarrow S$ be a generating mapping of a semigroup $S$, and let $s \in S$. We say that the cluster word $(L, \ell)$ over $A$ is recognized by the pair $(\varphi, s)$ if there is a mapping $g: \operatorname{step}(L) \rightarrow \mathfrak{F}(s)$ satisfying: 
(R.1) $g(\min L)=(1, s)$;

(R.2) $g(\max L)=(s, 1)$;

(R.3) if $p_{1} \prec p_{2}$ in $L$, then $g\left(p_{1}\right) \stackrel{\varphi\left(\ell\left(p_{1}\right)\right)}{\longrightarrow} g\left(p_{2}\right)$ is an edge of $\mathcal{T}(s)$;

(R.4) if $p$ is a stationary point of $L$ then, for every $q \in \mathfrak{F}(s)$, the set $g^{-1}(q)$ is left cofinal at $p$ if and only if it is right cofinal at $p$.

If such conditions are satisfied, then we say that $(L, \ell)$ is $g$-recognized by the pair $(\varphi, s)$. We also say that $(L, \ell)$ is recognized by the pair $(\varphi, s)$ if it is $g$-recognized, for some $g$. Finally, when $(L, \ell)$ is $g$-recognized by $(\varphi, s)$, we define $F_{g}$ to be the function from stat $(L)$ to the power set $\mathcal{P}(\mathfrak{F}(s))$ such that

$$
\begin{aligned}
F_{g}(p) & =\left\{q \in \mathfrak{F}(s): g^{-1}(q) \text { is left cofinal at } p\right\} \\
& =\left\{q \in \mathfrak{F}(s): g^{-1}(q) \text { is right cofinal at } p\right\}
\end{aligned}
$$

for every stationary point $p$ of $L$ (cf. (R.4)).

Remark 8.3. If $f:\left(L^{\prime}, \ell^{\prime}\right) \rightarrow(L, \ell)$ is an isomorphism of cluster words over $A$ and if $(L, \ell)$ is $g$-recognized by $(\varphi, s)$, then $\left(L^{\prime}, \ell^{\prime}\right)$ is $(g \circ f)$-recognized by $(\varphi, s)$. Hence, the property of $(L, \ell)$ being recognized by $(\varphi, s)$ is invariant under isomorphism of cluster words.

The next lemma, concerning the function $F_{g}$, will be applied in Section 11.

Lemma 8.4. Consider a cluster word $(L, \ell)$ over $A$, recognized by the pair $(\varphi, s)$, where $\varphi: A \rightarrow S$ is a generating mapping of a finite semigroup $S$ and $s \in S$. Suppose that the order topology of $L$ is metrizable. Let $p$ be a stationary point of $L$. Then the following properties hold:

(a) if $P$ is a subset of $\mathfrak{F}(s)$ such that $F_{g}^{-1}(P)$ is cofinal at $p$, then $P \subseteq F_{g}(p)$

(b) there is an open interval I containing $p$ such that $F_{g}(q) \subseteq F_{g}(p)$ for every stationary point $q$ in $I$.

Proof: (a) Without loss of generality, suppose that $F_{g}^{-1}(P)$ is left cofinal at $p$. Then, taking into account that $L$ is metrizable, there is a strictly increasing sequence $\left(p_{n}\right)_{n}$ of stationary points of $L$ converging to $p$ such that $F_{g}\left(p_{n}\right)=P$ for all $n$. Consider a metric $d$ on $L$ inducing the order topology of $L$. For each $k \geq 1$, let ] $q_{k}, p$ [ be an open interval of $L$ such that $d\left(q_{k}, p\right)<\frac{1}{k}$. Then, there is $n_{k}$ such that $\left.p_{n_{k}} \in\right] q_{k}, p[$. Let $\left(s_{1}, s_{2}\right) \in P$. By the definition of $F_{g}$, we know that $g^{-1}\left(s_{1}, s_{2}\right)$ is left cofinal at $p_{n_{k}}$. Therefore, there is in the interval $] q_{k}, p_{n_{k}}$ [ a step point belonging to $g^{-1}\left(s_{1}, s_{2}\right)$. Since $] q_{k}, p_{n_{k}}[\subseteq] q_{k}, p[$, this proves that 
$g^{-1}\left(s_{1}, s_{2}\right)$ is left cofinal at $p$, thus $\left(s_{1}, s_{2}\right) \in F_{g}(p)$. We have therefore proved that the inclusion $P \subseteq F_{g}(p)$ holds.

(b) Let $\mathcal{P}$ be the set of subsets $P$ of $\mathfrak{F}(s)$ such that $F_{g}^{-1}(P)$ is not cofinal at $p$. For each $P \in \mathcal{P}$, there is an open interval $I_{P}$ containing $p$ such that $F_{g}^{-1}(P) \cap I_{P}=\emptyset$. Because $S$ is a finite semigroup, the set $\mathcal{P}$ is finite, and so $I=\bigcap_{P \in \mathcal{P}} I_{P}$ is an open interval of $L$ containing $p$. Let $q$ be a stationary point in $I$. Let $R=F_{g}(q)$. Suppose $R \in \mathcal{P}$. Then $q \in I_{R}$. But we also have $q \in F_{g}^{-1}(R)$, which leads to $F_{g}^{-1}(R) \cap I_{R} \neq \emptyset$, a contradiction with the definition of $I_{R}$. This shows that $R \notin \mathcal{P}$. We then deduce from part (a) of the lemma that $R \subseteq F_{g}(p)$.

The main results in this section are about cluster words defined by elements of $\bar{\Omega}_{A} \mathrm{~A}$, but in the next proposition we embrace without additional effort all pseudovarieties closed under concatenation.

Proposition 8.5. Consider a pseudovariety $\mathrm{V}$ closed under concatenation. Given $w \in \bar{\Omega}_{A} \mathrm{~V}$, and a generating mapping $\varphi: A \rightarrow S$ of a semigroup $S$ of $\mathrm{V}$, let $s=\varphi \vee(w)$. Consider the mapping $g_{w, \varphi}: \operatorname{step}(\mathfrak{L}(w)) \rightarrow$ $\mathfrak{F}(s)$ such that $g_{w, \varphi}(u, v)=(\varphi \vee(u), \varphi \vee(v))$ for every step point $(u, v)$ of $\mathfrak{L}(w)$. Then the cluster word $\mathfrak{L}_{c}(w)$ is $g_{w, \varphi}$-recognized by $(\varphi, s)$.

Proof: The conditions (R.1)-(R.2) in Definition 8.2 for $g_{w, \varphi}$-recognition by $(\varphi, s)$ are clearly satisfied, and (R.3) follows directly from Proposition $6.7(\mathrm{a})$.

Let us check condition (R.4). Let $(x, y) \in \mathfrak{F}(s)$ be such that $g_{w, \varphi}^{-1}(x, y)$ is left cofinal at $p$. Then, there is a strictly increasing sequence $\left(u_{n}, v_{n}\right)_{n \geq 1}$ of step points belonging to $g_{w, \varphi}^{-1}(x, y)$ converging in $\mathfrak{F}(w)$ to an element $(u, v)$ of $p$. Since $\left(\varphi \vee\left(u_{n}\right), \varphi \vee\left(v_{n}\right)\right)=(x, y)$ for every $n \geq 1$, we also have $(\varphi \vee(u), \varphi \vee(v))=(x, y)$, by continuity of $\varphi \vee$. By the dual of Proposition 7.13 , there is a strictly decreasing sequence $\left(u_{n}^{\prime}, v_{n}^{\prime}\right)_{n \geq 1}$ of step points converging to $(u, v)$ in $\mathfrak{F}(w)$. In particular, by continuity of $\varphi \vee$, for all sufficiently large $n$, we have $\left(\varphi \vee\left(u_{n}^{\prime}\right), \varphi \vee\left(v_{n}^{\prime}\right)\right)=(x, y)$, and so $g_{w, \varphi}^{-1}(x, y)$ is right cofinal at $p$. Dually, if $g_{w, \varphi}^{-1}(x, y)$ is right cofinal at $p$ then $g_{w, \varphi}^{-1}(x, y)$ is left cofinal at $p$. This establishes condition (R.4).

In the case of unambiguous aperiodic semigroups, we have a converse for Proposition 8.5, as stated in the next theorem.

Theorem 8.6. Let $w \in \bar{\Omega}_{A} \mathrm{~A}$, and consider a generating mapping $\varphi: A \rightarrow$ $S$ of a finite aperiodic unambiguous semigroup $S$. Then $\varphi_{\mathrm{A}}(w)=s$ if and only if $\mathfrak{L}_{c}(w)$ is recognized by $(\varphi, s)$. 
We defer the proof of Theorem 8.6 to Section 11 (but note that the direct implication in Theorem 8.6 is an immediate application of Proposition 8.5). Meanwhile, we use it to prove the following main result.

Theorem 8.7. Let $u, v \in \bar{\Omega}_{A} \mathrm{~A}$. Then $u=v$ if and only if the cluster words $\mathfrak{L}_{c}(u)$ and $\mathfrak{L}_{c}(v)$ are isomorphic.

Proof: The isomorphism of cluster words is clearly necessary to have $u=$ $v$. Conversely, suppose $\mathfrak{L}_{c}(u)$ and $\mathfrak{L}_{c}(v)$ are isomorphic. Let $\varphi: A \rightarrow S$ be a generating mapping of a finite unambiguous aperiodic semigroup $S$. Take $s=\varphi_{\mathrm{A}}(u)$. Then $\mathfrak{L}_{c}(u)$ is recognized by $(\varphi, s)$, according to the direct implication in Theorem 8.6. But then $\mathfrak{L}_{c}(v)$ is also recognized by $(\varphi, s)\left(\mathrm{cf}\right.$. Remark 8.3). Hence, we have $s=\varphi_{\mathrm{A}}(v)$ by the converse implication in Theorem 8.6. By Proposition 2.1, this establishes $u=$ $v$.

\section{Stabilizers}

Given a semigroup $S$ and $s \in S$, we say that an element $x$ of $S^{I}$ stabilizes $s$ on the right if $s x=s$; the set $\operatorname{rstab}(s)$ of all such $x$ constitutes a submonoid of $S^{I}$ and is called the right stabilizer of $s$. One defines dually the elements that stabilize $s$ on the left, which form a $\operatorname{submonoid} \operatorname{lstab}(s)$ of $S^{I}$, called the left stabilizer of $s$.

An application of the following result will be required in the sequel.

Theorem 9.1. Let $S$ be an equidivisible profinite semigroup which is finitely cancelable. Let $u \in S$. Let $g$ be an element of the monoid $\operatorname{rstab}(u)$ or of the monoid $\operatorname{Istab}(u)$. If $g$ is regular within that monoid, then $g=g^{2}$.

In Theorem 9.1, the hypothesis that $S$ is finitely cancelable is not superfluous: if $G$ is a group, then the semigroup $G^{0}$ obtained from $G$ by adjoining a zero is equidivisible, and $G^{0}$ is the left and right stabilizer of zero.

We should mention that we do not know of any other examples of equidivisible profinite semigroups that are finitely cancelable other than free pro- $\mathrm{V}$ semigroups, where $\mathrm{V}$ is a pseudovariety with suitable closure properties. For such semigroups, Theorem 9.1 follows from more general results in [39, Theorem 13.1]. Nevertheless, since our results apply to all equidivisible profinite semigroups that are finitely cancelable, we present a proof of Theorem 9.1 which may be of independent interest.

As a first step we have the following simple statement.

Lemma 9.2. Let $x$ and $y$ be $\mathcal{D}$-equivalent elements of a stable semigroup $S$. If $y x=x$ then $y=y^{2}$. 
Proof: Since $y x=x$ and $y$ are in the same $\mathcal{D}$-class and $S$ is stable, we must have $y \mathcal{R} x$. Therefore, $y=x u$ for some $u \in S^{I}$, so $y^{2}=y x u=$ $x u=y$.

We proceed with an auxiliary lemma.

Lemma 9.3. Let $S$ be an equidivisible profinite semigroup which is finitely cancelable. Let $g, w \in S$ be such that $g w=w$. If $(x, y) \in \mathfrak{F}(w)$ is a step point satisfying $\left(g^{\omega}, w\right) \leq(x, y)$, then $g x=x$.

Proof: Note that $\left(g^{\omega}, w\right) \leq(x, y)$ implies $x \leq_{\mathcal{R}} g^{\omega}$, thus $x=g^{\omega} x$, a fact that we shall use along the proof.

By equidivisibility, $(x, y)$ and $(g x, y)$ are comparable in $\mathfrak{F}(w)$. Suppose first that $(g x, y) \leq(x, y)$. Then there is $t \in S^{I}$ such that $g x t=x$ and $t y=y$. It suffices to show that $t=1$. The equality $g x t=x$ entails $x=$ $g^{n} x t^{n}$ for every $n \geq 1$, thus $x=g^{\omega} x t^{\omega}=x t^{\omega}$. And since $t y=y$, we conclude that $t^{\omega}$ stabilizes $(x, y)$ in $\mathcal{T}(S)$. Because $(x, y)$ is a step point, Proposition 7.5 implies that $t=1$.

Suppose next that $(x, y) \leq(g x, y)$. Then $\left(g^{\omega-1} x, y\right) \leq\left(g^{\omega} x, y\right)=$ $(x, y)$ (cf. Remark 4.6) and, by the preceding case, we deduce that $g^{\omega-1} x=x$. With a left multiplication by $g$ on both sides of the latter equality, we obtain $x=g^{\omega} x=g x$, as desired.

Proof of Theorem 9.1: It suffices to consider the case where $g$ is an element of $\operatorname{Istab}(u)$, as the other case is dual.

We first establish the theorem when $g$ is a group element, that is, $g=g^{\omega+1}$.

Let $R$ be the set of elements $(\alpha, \beta)$ of $\mathfrak{F}(u)$ such that $\alpha \mathcal{R} g$. Note that $R$ is nonempty, indeed $(g, u) \in R$. The set $R$ is closed, whence compact, and so by continuity of $\chi$, the image $\chi(R)$ is also compact, whence closed. Therefore, by completeness of $\mathfrak{L}(u)$ (cf. Proposition 4.5), the closed set $\chi(R)$ has a maximum $p=(x, y) / \sim$. Let us observe that, since two $\sim$-equivalent elements must have $\mathcal{R}$-equivalent first components, the inclusion $\chi^{-1}(p) \subseteq R$ holds. Moreover, since $g=g^{\omega+1}$, we have $\left(g^{\omega}, u\right) \in$ $R$, thus $\left(g^{\omega}, u\right) \leq(x, y)$ by definition of $p$.

Suppose first that $p$ is a step point. Then $g x=x$ by Lemma 9.3. As $x \mathcal{R} g$, we conclude that $g=g^{2}$ by Lemma 9.2 .

If $p$ is stationary, then, by Theorem 7.4 , there is a net $\left(x_{i}, y_{i}\right)_{i \in I}$ of step points converging in $\mathfrak{L}(u)$ to $p$ and such that $p<\left(x_{i}, y_{i}\right)$ for all $i \in I$. By compactness, taking a subnet, we may assume that $\left(x_{i}, y_{i}\right)_{i \in I}$ converges in $\mathfrak{F}(u)$ to some element $\left(x^{\prime}, y^{\prime}\right)$ of $p$. As $\left(g^{\omega}, u\right)<\left(x_{i}, y_{i}\right)$, we deduce from Lemma 9.3 that $g x_{i}=x_{i}$ for every $i \in I$. Taking limits, it follows that $g x^{\prime}=x^{\prime}$. Since $\left(x^{\prime}, y^{\prime}\right) \in p$, we have $x \mathcal{R} x^{\prime}$, whence $g \mathcal{R} x^{\prime}$, and we again deduce that $g=g^{2}$ by Lemma 9.2. 
We have thus concluded the proof for the case where $g$ is a group element of $S$. Let us now suppose that $g$ is regular within Istab $(u)$. Then there is $h \in \operatorname{Istab}(u)$ such that $g=g h g$ and $h=h g h$. Since $h u=$ $g u$, by equidivisibility we know that $h$ and $g$ are $\mathcal{R}$-comparable in $S$. We actually have $h \mathcal{R} g$ in $S$, by stability of $S$. Let $z \in S^{I}$ be such that $h=g z$. Then $g=g^{2} z g$, and therefore $g \mathcal{R} g^{2}$. This shows that $g$ is a group element of $S$, and so, as we are in the case already proved, we get $g=g^{2}$.

A semigroupoid $S$ is trivial if, for any two vertices $p, q \in S$, there is at most one edge $p \rightarrow q$.

Corollary 9.4. Let $S$ be an equidivisible profinite semigroup which is finitely cancelable. For every $p \in \mathfrak{L}(S)$, the ideal $\mathcal{K}_{p}$ is a trivial category.

Proof: Let $(u, v) \stackrel{s}{\rightarrow}(x, y)$ and $(u, v) \stackrel{t}{\rightarrow}(x, y)$ be edges of $\mathcal{K}_{p}$. The proof amounts to showing that $s=t$. There is an edge $(x, y) \stackrel{z}{\rightarrow}(u, v)$ in $\mathcal{K}_{p}$. In particular, $s z$ and $(s z)^{2}$ label loops in $\mathcal{K}_{p}$ at vertex $(u, v)$. Hence, $s z$ and $(s z)^{2}$ belong to $J_{p}$, and so $s z$ is a group element of $S$ stabilizing $u$ on the right. We then deduce from Theorem 9.1 that $s z$ is an idempotent of $J_{p}$, denoted by $e$, which stabilizes $(u, v)$. Similarly, $t z$ is an idempotent of $J_{p}$ stabilizing $(u, v)$. It follows from Lemma 5.3 that $s z=t z=e$. Symmetrically, we have $z s=z t=f$, with $f$ an idempotent of $J_{p}$. As $s, t, z$ belong to $J_{p}$, this shows that $s=t$.

For a compact semigroup $S$ and $p \in \mathfrak{L}(S)$, let $U_{p}$ be the union of the maximal subgroups of $J_{p}$. The next proposition, whose proof relies on Theorem 9.1, should be compared with Proposition 5.9. We show that $U_{p}$ parameterizes in a natural way the class $p$, without assuming aperiodicity, but assuming equidivisibility and finite cancelability.

Proposition 9.5. Let $S$ be an equidivisible profinite semigroup which is finitely cancelable. Let $p \in \mathfrak{L}(S)$ be a stationary point. Then we have a bijection $\nu_{p}: U_{p} \rightarrow p$, defined as follows:

(a) for each idempotent $e \in J_{p}$, fix an element $\left(u_{e}, v_{e}\right)$ of $p$ stabilized by $e$;

(b) to each $g$ in the maximal subgroup $H_{e}$ of $S$ containing e, associate the element $\nu_{p}(g)=\left(u_{e} g, g^{\omega-1} v_{e}\right)$ of $p$.

Proof: We first verify that the function $\nu_{p}$ is well defined. Corollary 5.6 guarantees that every idempotent $e \in J_{p}$ stabilizes some $\left(u_{e}, v_{e}\right) \in p$. If $g \in H_{e}$, then $\left(u_{e} g, g^{\omega-1} v_{e}\right) \stackrel{g^{\omega-1}}{\longrightarrow}\left(u_{e}, v_{e}\right)$ and $\left(u_{e}, v_{e}\right) \stackrel{g}{\rightarrow}\left(u_{e} g, g^{\omega-1} v_{e}\right)$ are edges of $\mathcal{T}(S)$, whence $\left(u_{e} g, g^{\omega-1} v_{e}\right) \sim\left(u_{e}, v_{e}\right)$. It follows that $\nu_{p}$ is indeed well defined. 
We next show that $\nu_{p}$ is injective. If $g \in U_{p}$ then $g^{\omega}$ is an idempotent of $J_{p}$ stabilizing $\nu_{p}(g)=\left(u_{g^{\omega}} g, g^{\omega-1} v_{g^{\omega}}\right)$. Hence, by Lemma 5.3, if $\nu_{p}(g)=\nu_{p}(h)$, then $g^{\omega}=h^{\omega}$, thus $g, h$ belong to the same maximal subgroup and $u_{g^{\omega}} g=u_{g^{\omega}} h$. The latter is equivalent to $u_{g^{\omega}}=u_{g^{\omega}} h g^{\omega-1}$, and so, by Theorem 9.1, we have $h g^{\omega-1}=g^{\omega}$. This means that $g=h$, thereby showing that $\nu_{p}$ is injective.

It remains to show that $\nu_{p}$ is surjective. Let $(u, v)$ be an arbitrary element of $p$ and let $e$ be an idempotent in $J_{p}$ stabilizing $(u, v)$. Since $p$ is the set of vertices of a strongly connected component of $\mathcal{T}(S)$, there is some edge $\left(u_{e}, v_{e}\right) \stackrel{t}{\rightarrow}(u, v)$, and whence also an edge $\left(u_{e}, v_{e}\right) \stackrel{\text { ete }}{\rightarrow}(u, v)$. By Lemma 5.2, it follows that ete $\in H_{e}$. Hence, we must have $\nu_{p}($ ete $)=$ $(u, v)$.

Note that, under the conditions of Proposition 9.5, the set $p_{e}$ of elements of $p$ stabilized by $e$ is precisely $\nu_{p}\left(H_{e}\right)$.

\section{A characterization of the $\mathcal{J}$-class associated to a $\sim$-class}

Let $S$ be an equidivisible compact semigroup, $w \in S$ and $p \in \mathfrak{L}(w)$. We define a subset $L_{p}$ of $S^{I}$, depending only on $p$, as follows. Take an arbitrary strictly increasing sequence $p_{1}<p_{2}<\cdots$ converging to $p$ in $\mathfrak{L}(w)$ - if such a sequence does not exist, for instance, if $p$ has a predecessor, then take $L_{p}=\emptyset$. For each $m \geq 1$ and $n>m$, let $t_{m, n}$ be a transition from $p_{m}$ to $p_{n}$. For fixed $m \geq 1$, let $t_{m}$ be an accumulation point of the sequence $\left(t_{m, n}\right)_{n>m}$. If $t$ is an accumulation point of the sequence $\left(t_{m}\right)_{m}$ then $t \in L_{p}$, and every element of $L_{p}$ is obtained by this process, the sequence $p_{1}<p_{2}<\cdots$ being allowed to change. Dually, taking strictly decreasing sequences converging to $p$, we define a subset $R_{p}$ of $S$ associated with $p$.

Theorem 10.1. Let $S$ be an equidivisible compact semigroup. For every $p \in \mathfrak{L}(S)$, the sets $L_{p}$ and $R_{p}$ are contained in $J_{p}$.

Proof: Let $w \in S$ be such that $p \in \mathfrak{L}(w)$, and suppose that $\left(p_{n}\right)_{n}$ is a strictly increasing sequence of elements of $\mathfrak{L}(w)$ converging to $p$. For each $m \geq 1$ and $n>m$, let $t_{m, n}$ be a transition from $p_{m}$ to $p_{n}$, and let $t_{m}$ be an accumulation point of the sequence $\left(t_{m, n}\right)_{n}$. Finally, let $t$ be an accumulation point of the sequence $\left(t_{m}\right)_{m}$. The proof that $L_{p}$ is contained in $J_{p}$ is concluded once we show that $t \in J_{p}$.

We first claim that, for every fixed $m \geq 1, t_{m}$ is a transition from $p_{m}$ to $p$. For each $n>m$, choose $\left(u_{n}, v_{n}\right) \in p_{m}$ and $\left(x_{n}, y_{n}\right) \in p_{n}$ such that $u_{n} t_{m, n}=x_{n}$ and $v_{n}=t_{m, n} y_{n}$. By compactness, the sequence 
$\left(t_{m, n}, u_{n}, v_{n}, y_{n}\right)_{n}$ has some subnet $\left(t_{m, n_{k}}, u_{n_{k}}, v_{n_{k}}, y_{n_{k}}\right)_{n_{k}}$ such that $t_{m}=$ $\lim _{k} t_{m, n_{k}}$ and the nets $\left(u_{n_{k}}\right)_{k},\left(v_{n_{k}}\right)_{k}$, and $\left(y_{n_{k}}\right)_{k}$ converge, respectively to some $u, v$, and $y$. Since $\left(u_{n_{k}}, t_{m, n_{k}} y_{n_{k}}\right)=\left(u_{n_{k}}, v_{n_{k}}\right) \in p_{m}$ and $\left(u_{n_{k}} t_{m, n_{k}}, y_{n_{k}}\right)=\left(x_{n_{k}}, y_{n_{k}}\right) \in p_{n_{k}}$, it follows from Proposition 4.3 that $(u, v)=\left(u, t_{m} y\right) \in p_{m}$ and $\left(u t_{m}, y\right) \in p$, which proves the claim.

Since $\left(p_{m}\right)_{m}$ converges to $p$ and $t$ is an accumulation point of the sequence $\left(t_{m}\right)_{m}$, it follows again from Proposition 4.3 that $t$ is a transition from $p$ to $p$.

Choose $z \in J_{p}$ such that there is a loop $\left(u t_{m}, y\right) \stackrel{z}{\rightarrow}\left(u t_{m}, y\right)$. Since $(u, v)<\left(u t_{m}, y\right)$ and $(u, v) \stackrel{t_{m}}{\longrightarrow}\left(u t_{m}, y\right)$ is an edge of $\mathcal{T}(S)$, Lemma 4.7 yields that $z$ is a suffix of $t_{m}$. This holds for every $m \geq 1$, whence $z$ is a suffix of $t$. As we have already shown that $t$ is a transition from $p$ to $p$, we deduce that $t \in J_{p}$ by Lemma 5.2. Hence we have $L_{p} \subseteq J_{p}$ and dually $R_{p} \subseteq J_{p}$.

Corollary 10.2. Let $S$ be an equidivisible profinite semigroup which is finitely cancelable. Let $w \in S$. Suppose that $\left(u_{n}, v_{n}\right)_{n}$ is a strictly increasing sequence in $\mathfrak{F}(w)$ converging to a stationary point $(u, v)$. For each pair $m<n$, let $t_{m, n} \in S$ be a transition $\left(u_{m}, v_{m}\right) \rightarrow\left(u_{n}, v_{n}\right)$. Then, for each $m$, the sequence $\left(t_{m, n}\right)_{n}$ converges to the unique transition from $\left(u_{m}, v_{m}\right)$ to $(u, v)$. Moreover, the sequence $\left(t_{m}\right)_{m}$ converges to the label of the only loop at the vertex $(u, v)$ in the trivial category $\mathcal{K}_{p}$, where $p=(u, v) / \sim$.

Proof: Every accumulation point of the sequence $\left(t_{m, n}\right)_{n}$ labels an edge from $\left(u_{m}, v_{m}\right)$ to $(u, v)$. But there is only one such edge, by Proposition 7.6. Since we are dealing with a compact space, this implies that $\left(t_{m, n}\right)_{n}$ converges to some element $t_{m}$. To conclude the proof, it suffices to show that every accumulation point $t$ of the sequence $\left(t_{m}\right)_{m}$ labels the same loop at vertex $(u, v)$. By the definition of $L_{p}$, we have $t \in L_{p}$, whence $t \in J_{p}$ by Theorem 10.1. Moreover, in $\mathcal{T}(w)$ the sequence of edges $\left(u_{m}, v_{m}\right) \stackrel{t_{m}}{\rightarrow}(u, v)$ admits the loop $(u, v) \stackrel{t}{\rightarrow}(u, v)$ as an accumulation point. Since $t \in J_{p}$, this loop belongs to $\mathcal{K}_{p}$ by Corollary 5.4. By Corollary 9.4 , there is only one loop of $\mathcal{K}_{p}$ at $(u, v)$. Therefore, every accumulation point of $\left(t_{m}\right)_{m}$ is the label of that loop.

In some cases, Theorem 10.1 can be strengthened, as seen next.

Theorem 10.3. Let $A$ be a finite alphabet, and let $\mathrm{V}$ be a pseudovariety closed under concatenation. Then $L_{p}=J_{p}=R_{p}$ for every stationary point $p \in \mathfrak{L}\left(\bar{\Omega}_{A} \bigvee\right)$.

Proof: According to Theorem 10.1, it remains to prove that $J_{p}$ is contained in $L_{p}$ and in $R_{p}$. By symmetry, it suffices to prove that $J_{p} \subseteq L_{p}$. 
Let $\tau$ be an element of $J_{p}$. Then by Proposition 5.7 there are elements $(u, v)$ and $\left(u^{\prime}, v^{\prime}\right)$ of $p$ such that $(u, v) \stackrel{\tau}{\rightarrow}\left(u^{\prime}, v^{\prime}\right)$ is an edge of $\mathcal{K}_{p}$. According to Proposition 7.13, there are strictly increasing sequences $\left(q_{n}\right)_{n}$ and $\left(q_{n}^{\prime}\right)_{n}$ of step points of $\mathfrak{F}(w)$ converging to $(u, v)$ and $\left(u^{\prime}, v^{\prime}\right)$, respectively. We may define recursively a strictly ascending sequence of step points $p_{n}$ as follows:

- $p_{1}=q_{1}$;

- if $n>1$ is even, then $p_{n}$ is the smallest term of the sequence $\left(q_{n}^{\prime}\right)_{n}$ belonging to $] p_{n-1}, p[$;

- if $n>1$ is odd, then $p_{n}$ is the smallest term of the sequence $\left(q_{n}\right)_{n}$ belonging to $] p_{n-1}, p[$;

For each $m \geq 1$ and each $n \geq m$ let $t_{m, n}$ be the unique transition from $p_{2 m-1}$ to $p_{2 n}$. Let $t_{m}$ be an accumulation point of the sequence $\left(t_{m, n}\right)_{n \geq m}$. Since $\left(p_{2 n}\right)_{n}$ converges to $\left(u^{\prime}, v^{\prime}\right)$, the pseudoword $t_{m}$ labels an edge from $p_{2 m-1}$ to $\left(u^{\prime}, v^{\prime}\right)$. Let $t$ be an accumulation point of the sequence $\left(t_{m}\right)_{m}$. Since $\left(p_{2 m-1}\right)_{m}$ converges to $(u, v)$, the pseudoword $t$ labels an edge from $(u, v)$ to $\left(u^{\prime}, v^{\prime}\right)$. By the definition of $L_{p}$, we have $t \in L_{p}$. It follows that $t \in J_{p}$ by Theorem 10.1. By Corollary 9.4, the category $\mathcal{K}_{p}$ is trivial, whence $t=\tau$, thus proving that $\tau \in L_{p}$.

\section{Proof of Theorem 8.6}

Throughout this section, when not explicitly stated, we consider $w$ to be an element of $\bar{\Omega}_{A} \mathrm{~A}$, where $A$ is a finite alphabet, and $\varphi: A \rightarrow S$ to be a generating mapping of a finite aperiodic semigroup $S$. We also take $s=\varphi_{\mathrm{A}}(w)$.

Consider a mapping $g: \operatorname{step}(\mathfrak{L}(w)) \rightarrow \mathfrak{F}(s)$. From hereon, we assume that $\mathfrak{L}_{c}(w)$ is $g$-recognized by the pair $(\varphi, s)$. In particular, all properties of Definition 8.2 are fulfilled when $(L, \ell)=\mathfrak{L}_{c}(w)$.

Definition 11.1 (g-projection). Let $x$ and $y$ be two step points of $\mathfrak{L}(w)$ such that $x \leq y$. By Propositions 7.6 (in case $x<y$ ) and 7.5 (in case $x=y$ ), there is a unique edge in $\mathcal{T}(w)$ from $x$ to $y$. Let $t$ be its label, which is 1 if $x=y$. If $g(x) \stackrel{\varphi_{\mathrm{A}}(t)}{\longrightarrow} g(y)$ is an edge of $\mathcal{T}(s)$, then we say that the edge $x \stackrel{t}{\rightarrow} y$ is $g$-projected (to $g(x) \stackrel{\varphi_{\mathrm{A}}(t)}{\longrightarrow} g(y)$ ).

The unique edge from $x$ to $y$ will sometimes be denoted simply by $x \rightarrow$ $y$, without reference to the label.

Remark 11.2. Let $x, y$, and $z$ be step points such that $x \leq y \leq z$. If $x \rightarrow y$ and $y \rightarrow z$ are $g$-projected, then $x \rightarrow z$ is $g$-projected. 
Given two step points $x$ and $y$, write $x \prec \prec y$ if $x \leq y$ and the interval $[x, y]$ is finite.

Remark 11.3. If $x \prec \prec y$, then $x \rightarrow y$ is $g$-projected.

Let $\approx$ be the equivalence relation on $\operatorname{step}(\mathfrak{L}(w))$ generated by $\prec \prec$, that is, $x \approx y$ if and only $x \prec \prec y$ or $y \prec \prec x$. The $\approx$-class of $x$ is denoted $[x]_{\approx}$. Note that $w \in A^{+}$if and only if $(1, w) \approx(w, 1)$. Let $\mathcal{O}_{w}$ be a subset of $\operatorname{step}(\mathfrak{L}(w))$ such that each $\approx$-class contains exactly one element of $\mathcal{O}_{w}$, with the additional restriction that if $w \notin A^{+}$then we have

$$
\mathcal{O}_{w} \cap[(1, w)]_{\approx}=\{(1, w)\} \quad \text { and } \quad \mathcal{O}_{w} \cap[(w, 1)]_{\approx}=\{(w, 1)\} .
$$

Definition 11.4 (Bridges). A bridge in $\mathfrak{L}(w)$, with respect to the mapping $g$, is a nonempty open interval $I$ of $\mathfrak{L}(w)$ such that, for every pair of step points $x, y$ of $I$, with $x<y$, the edge $x \rightarrow y$ is $g$-projected. A special bridge in $\mathfrak{L}(w)$ is a bridge of the form $\left[X, Y\left[\right.\right.$, with $X, Y \in \mathcal{O}_{w}$ such that $X<Y$.

Notice that every nonempty interval contained in a bridge is also a bridge, and that special bridges are clopen intervals.

Lemma 11.5. Let $\mathcal{F}$ be a nonempty family of special bridges of $\mathfrak{L}(w)$. If $\bigcup \mathcal{F}$ is a closed interval, then $\bigcup \mathcal{F}$ is a special bridge.

Before proving Lemma 11.5, we remark that the hypothesis that the union $\bigcup \mathcal{F}$ is closed cannot be removed. Indeed, consider a case where we have a strictly increasing sequence $\left(q_{n}\right)_{n}$ of stationary points converging to a stationary point $q$ (cf. Example 8.1). Between $q_{n}$ and $q_{n+1}$ pick an element $p_{n}$ of $\mathcal{O}_{w}$. Then the union of the special bridges $\left[\min \mathfrak{L}(w), p_{n}[\right.$ is $[\min \mathfrak{L}(w), q[$, which is not a special bridge.

Proof of Lemma 11.5: Since $\bigcup \mathcal{F}$ is a compact set having an open cover by the elements of $\mathcal{F}$, we have $\bigcup \mathcal{F}=\bigcup \mathcal{F}^{\prime}$ for some finite subfamily $\mathcal{F}^{\prime}$ of $\mathcal{F}$. Then, for some $n \geq 1$, we may assume that there are elements $X_{1}<X_{2}<\cdots<X_{n}$ and $Y_{1}<Y_{2}<\cdots<Y_{n}$ of $\mathcal{O}_{w}$ such that

$$
\mathcal{F}^{\prime}=\left\{\left[X_{k}, Y_{k}[: 1 \leq k \leq n\} .\right.\right.
$$

Consider the set

$$
Z=\left\{X_{i}: 1 \leq k \leq n\right\} \cup\left\{Y_{i}: 1 \leq k \leq n\right\},
$$

and let $Z_{1}<Z_{2}<\cdots<Z_{m}$ be the elements of $z$. Notice that $m \geq 2$. For each $k \in\{1, \ldots, m-1\}$, denote by $I_{k}$ the interval $\left[Z_{k}, Z_{k+1}[\right.$. In case $Z_{k}=X_{j}$ for some $j$, we have $Z_{k+1} \leq Y_{j}$, and so $I_{k}$ is a special bridge contained in the special bridge $\left[X_{j}, Y_{j}\left[\right.\right.$. If $Z_{k}=Y_{j}$ for some $j$, 
then, since $Z_{k}<Z_{m}$, we must have $Z_{k} \in \bigcup \mathcal{F}$, whence $Z_{k} \in\left[X_{i}, Y_{i}\right.$ [ for some $i$. As $Z_{k}<Y_{i}$, we have $Z_{k+1} \leq Y_{i}$, and so $I_{k}$ is a special bridge contained in the special bridge $\left[X_{i}, Y_{i}[\right.$. Hence, the set

$$
\mathcal{F}^{\prime \prime}=\left\{I_{k}: 1 \leq k \leq m-1\right\}
$$

is a family of special bridges.

Let $U_{k}=\bigcup_{j=1}^{k} I_{j}$. Note that $U_{k}=\left[Z_{1}, Z_{k+1}[\right.$. We prove by induction on $k \in\{1, \ldots, m-1\}$ that $U_{k}$ is a special bridge. The initial step is trivial. Suppose that $U_{k}$ is a special bridge, for some $k \in\{1, \ldots, m-2\}$. To prove that $U_{k+1}$ is a special bridge, consider two step points $x, y$ in $U_{k+1}$ with $x<y$. We have to show that the edge $x \rightarrow y$ is $g$-projected. Using induction, it suffices to consider the case where $x \in U_{k}$ and $y \in$ $I_{k+1}$. Let $Z_{k+1}^{\prime}$ be the predecessor of the step point $Z_{k+1}$ in $\mathfrak{L}(w)$. Since $U_{k}=\left[Z_{1}, Z_{k+1}\left[\right.\right.$ and $I_{k+1}=\left[Z_{k+1}, Z_{k+2}\left[\right.\right.$, the edges $x \rightarrow Z_{k+1}^{\prime}$ and $Z_{k+1} \rightarrow y$ are $g$-projected, and the same is true obviously for the edge $Z_{k+1}^{\prime} \rightarrow Z_{k+1}$; hence, by Remark $11.2, x \rightarrow y$ is $g$-projected. This proves that $U_{k+1}$ is a special bridge, concluding the induction.

The result now follows because $U_{m-1}=\bigcup \mathcal{F}^{\prime \prime}=\bigcup \mathcal{F}^{\prime}=\bigcup \mathcal{F}$.

In the proof of the next lemma and in the sequel, we use the notation $\lambda(x)=\inf [x] \approx$ and $\rho(x)=\sup [x] \approx$, for a step point $x$. Note that $\lambda(x)$ is stationary unless $(1, w) \prec \prec x$, and $\rho(x)$ is stationary unless $x \prec \prec(w, 1)$. Also, when $r \in \operatorname{step}(\mathfrak{L}(w))$, the unique element of $\mathcal{O}_{w} \cap[r] \approx$ is denoted $\mathcal{O}_{w}(r)$.

Lemma 11.6. Let $X, Y \in \mathcal{O}_{w}$ be such that $X<Y$. Suppose that for every stationary point $p$ in $[X, Y[$ there is a special bridge containing $p$. Then $[X, Y[$ is a special bridge.

Proof: Let $P=[X, Y[\cap \operatorname{stat}(\mathfrak{L}(w))$. By hypothesis, for each $p \in P$ there is a special bridge $I_{p}$ containing $p$. Note that $I_{p} \cap[X, Y$ is also a special bridge containing $p$. Therefore, we may as well assume that $I_{p} \subseteq[X, Y[$. Let $U=\bigcup_{p \in P} I_{p}$. Then $U \subseteq[X, Y[$.

We claim that $U=[X, Y[$. As we trivially have $P \subseteq U$, we are reduced to showing that, for every $z \in[X, Y[\cap \operatorname{step}(\mathfrak{L}(w))$, we have $z \in U$.

If $\rho(X)<z<\lambda(Y)$ holds, then the stationary points $\rho(z)$ and $\lambda(z)$ belong to $\left[X, Y[\right.$. Either $z \in] \lambda(z), \mathcal{O}_{w}(z)\left[\right.$ or $z \in\left[\mathcal{O}_{w}(z), \rho(z)[\right.$. As ]$\lambda(z), \mathcal{O}_{w}(z)\left[\subseteq I_{\lambda(z)}\right.$ and $\left[\mathcal{O}_{w}(z), \rho(z)\left[\subseteq I_{\rho(z)}\right.\right.$, it follows that $z \in U$.

Suppose that $\rho(X) \geq z$. Then, since $X$ is the only point in $\mathcal{O}_{w}$ that belongs to $I_{\rho(X)} \cap\left[X, \rho(X)\left[\right.\right.$ and $z \in\left[X, \rho(X)\left[\right.\right.$, we have $z \in I_{\rho(X)}$. Similarly, if $\lambda(Y) \leq z$ then $z \in I_{\lambda(Y)}$. In both cases $z \in U$.

We proved that $U=[X, Y[$. By Lemma 11.5, the interval $[X, Y[$ is a special bridge. 
Let $p_{1}$ and $p_{2}$ be two elements of $\mathfrak{L}(w)$ such that $p_{1} \leq p_{2}$. If $p_{1}<p_{2}$, then, as written in Corollary 7.7, the set of transitions from $p_{1}$ to $p_{2}$ is contained in a $\mathcal{J}$-class $J_{p_{1}, p_{2}}$. In case $p_{1}=p_{2}$, let $J_{p_{1}, p_{2}}=J_{p_{1}}$. By a J-minimum transition from $p_{1}$ to $p_{2}$, we mean a transition from $p_{1}$ to $p_{2}$ belonging to $J_{p_{1}, p_{2}}$; this terminology is useful to unify both cases $p_{1}<p_{2}$ and $p_{1}=p_{2}$ in some of our arguments.

Lemma 11.7. Let $p_{1}, p_{2} \in \mathfrak{L}(w)$, with $p_{1} \leq p_{2}$. For every $\left(x_{1}, y_{1}\right) \in p_{1}$ and $\left(x_{2}, y_{2}\right) \in p_{2}$, the intersection $I$ of $J_{p_{1}, p_{2}}$ with the set of labels of edges from $\left(x_{1}, y_{1}\right)$ to $\left(x_{2}, y_{2}\right)$ is a singleton. If $t$ is the unique element of $I$, and if $e_{i}$ is the unique idempotent of $J_{p_{i}}$ that stabilizes $\left(x_{i}, y_{i}\right)$ (cf. Lemma 5.3), then $t=e_{1} t e_{2}$.

Proof: If $p_{1}<p_{2}$, then the lemma follows straightforwardly from Proposition 7.6. If $p_{1}=p_{2}=p$, then $J_{p_{1}, p_{2}}=J_{p}$. Since edges in $\mathcal{T}_{p}$ labeled by elements of $J_{p}$ are edges of $\mathcal{K}_{p}$ by Corollary 5.4, and since $\mathcal{K}_{p}$ is trivial by Corollary 9.4, we have also in this case that there is only one edge from $\left(x_{1}, y_{1}\right)$ to $\left(x_{2}, y_{2}\right)$ with label in $J_{p_{1}, p_{2}}$. Let $e_{i}$ and $t$ be as in the statement of the lemma. Because $e_{i}$ stabilizes $\left(x_{i}, y_{i}\right)$, we may consider the edge $\left(x_{1}, y_{1}\right) \stackrel{e_{1} t e_{2}}{\longrightarrow}\left(x_{1}, y_{2}\right)$, and so $t \mathcal{J} e_{1} t e_{2}$. By the already proved uniqueness, we must have $t=e_{1} t e_{2}$.

Definition 11.8 (J-bridge). A pair of elements $\left(p_{1}, p_{2}\right)$ of $\operatorname{stat}(\mathfrak{L}(w))$ is a $\mathcal{J}$-bridge with respect to $g$, if

(a) $p_{1} \leq p_{2}$,

(b) $F_{g}\left(p_{1}\right) \cap F_{g}\left(p_{2}\right) \neq \emptyset$,

(c) the elements of $\varphi_{\mathrm{A}}\left(J_{p_{1}}\right)$ are $\mathcal{J}$-equivalent to the elements of $\varphi_{\mathrm{A}}\left(J_{p_{2}}\right)$, and

(d) if $\tau$ is a $\mathcal{J}$-minimum transition from an element of $p_{1}$ to an element of $p_{2}$, then $\varphi_{\mathrm{A}}(\tau)$ is $\mathcal{J}$-equivalent to the elements of $\varphi_{\mathrm{A}}\left(J_{p_{1}}\right)$.

Note that $(p, p)$ is a J-bridge, whenever $p$ is a stationary point.

In the proof of the following proposition, the hypothesis that $S$ is an unambiguous aperiodic semigroup is essential.

Proposition 11.9. Suppose that $S$ is an unambiguous aperiodic semigroup and let $\left(p_{1}, p_{2}\right)$ be a J-bridge with respect to $g$. Suppose there are step points $z_{1}$ and $z_{2}$ such that $\left[z_{1}, p_{1}[\right.$ and $\left.] p_{2}, z_{2}\right]$ are bridges with respect to g. Let $\left(s_{1}, s_{2}\right) \in F_{g}\left(p_{1}\right) \cap F_{g}\left(p_{2}\right)$. Then there are step points $x_{1} \in$ $\left[z_{1}, p_{1}\left[\right.\right.$ and $\left.\left.x_{2} \in\right] p_{2}, z_{2}\right]$ such that $x_{1} \rightarrow x_{2}$ is g-projected to an idempotent loop of $\mathcal{T}(s)$ of the form $\left(s_{1}, s_{2}\right) \rightarrow\left(s_{1}, s_{2}\right)$.

Proof: Let $I_{1}=\left[z_{1}, p_{1}\left[\right.\right.$ and $\left.\left.I_{2}=\right] p_{2}, z_{2}\right]$. By the definition of $F_{g}\left(p_{i}\right)$, there are strictly monotone sequences $\left(r_{i, m}\right)_{m \geq 1}$ (increasing if $i=1$, 
decreasing if $i=2$ ) of step points in $I_{i} \cap g^{-1}\left(s_{1}, s_{2}\right)$, converging in $\mathfrak{F}(w)$ to elements $r_{i}$ of $p_{i}(i=1,2)$. Denote by $e_{i}$ the unique idempotent of $J_{p_{i}}$ that stabilizes $r_{i}$.

For each $n \geq m$, let $t_{1, m, n}$ be the label of the unique edge from $r_{1, m}$ to $r_{1, n}$, and let $t_{2, m, n}$ be the label of the unique edge from $r_{2, n}$ to $r_{2, m}$. By Corollary 10.2 and its dual, for each $i \in\{1,2\}$ the sequence $\left(t_{i, m, n}\right)_{n}$ converges to an element $t_{i, m}$, and in turn the sequence $\left(t_{i, m}\right)_{m}$ converges to $e_{i}$. Therefore, by continuity of $\varphi_{\mathrm{A}}$, for each $i \in\{1,2\}$ we may take $m_{i} \geq 1$ and $n_{i} \geq m_{i}$ for which we have

$$
\varphi_{\mathrm{A}}\left(e_{i}\right)=\varphi_{\mathrm{A}}\left(t_{i, m_{i}}\right)=\varphi_{\mathrm{A}}\left(t_{i, m_{i}, n_{i}}\right) .
$$

Our hypothesis yields that the edge $r_{1, m_{1}} \stackrel{t_{1, m_{1}, n_{1}}}{\longrightarrow} r_{1, n_{1}}$ is $g$-projected to the edge $\left(s_{1}, s_{2}\right) \stackrel{\varphi_{\mathrm{A}}\left(e_{1}\right)}{\longrightarrow}\left(s_{1}, s_{2}\right)$. In particular, $\left(s_{1}, s_{2}\right)$ is stabilized by $\varphi_{\mathrm{A}}\left(e_{1}\right)$. Similarly, $\left(s_{1}, s_{2}\right)$ is stabilized by $\varphi_{\mathrm{A}}\left(e_{2}\right)$. It follows that $\varphi_{\mathrm{A}}\left(e_{1}\right)=\varphi_{\mathrm{A}}\left(e_{2}\right)$, by Lemma 5.3 , since the finite semigroup $S$ is unambiguous.

By Lemma 11.7, the unique minimum $\mathcal{J}$-transition $\tau$ from $r_{1}$ to $r_{2}$ is such that $\tau=e_{1} \tau e_{2}$. By the definition of $\mathcal{J}$-bridge, we have $\varphi_{\mathrm{A}}\left(e_{1}\right) \mathrm{J}$ $\varphi_{\mathrm{A}}(\tau)$. On the other hand, $\tau \leq_{\mathcal{R}} e_{1}$ and $\tau \leq_{\mathcal{L}} e_{2}$. We deduce that $\varphi_{\mathrm{A}}\left(e_{1}\right) \mathcal{H} \varphi_{\mathrm{A}}(\tau)$ because $\varphi_{\mathrm{A}}\left(e_{1}\right)=\varphi_{\mathrm{A}}\left(e_{2}\right)$ and $S$ is stable. Therefore,

$$
\varphi_{\mathrm{A}}\left(e_{1}\right)=\varphi_{\mathrm{A}}(\tau)=\varphi_{\mathrm{A}}\left(e_{2}\right),
$$

since $S$ is aperiodic.

The unique edge (cf. Proposition 7.6) from $r_{1, m_{1}}$ to $r_{2, m_{2}}$, with label $\zeta$, is factorized by the edges $r_{1, m_{1}} \stackrel{t_{1, m_{1}}}{\longrightarrow} r_{1}, r_{1} \stackrel{\tau}{\rightarrow} r_{2}$, and $r_{2} \stackrel{t_{2, m_{2}}}{\longrightarrow} r_{2, m_{2}}$. It follows that $\zeta=t_{1, m_{1}} \tau t_{2, m_{2}}$. From (11.2) and (11.1) we get $\varphi_{\mathrm{A}}(\zeta)=$ $\varphi_{\mathrm{A}}\left(e_{1}\right)$. Therefore, $r_{1, m_{1}} \rightarrow r_{2, m_{2}}$ is $g$-projected to the idempotent loop $\left(s_{1}, s_{2}\right) \stackrel{\varphi_{\mathrm{A}}(\zeta)}{\longrightarrow}\left(s_{1}, s_{2}\right)$. Since $r_{i, m_{i}}$ is a step point in $I_{i}$, this concludes the proof.

We next show a pair of lemmas where the function $g$ does not appear, but which will be used later in this section in the proof of Theorem 8.6.

Lemma 11.10. Consider a pseudovariety $\mathrm{V}$ closed under concatenation. Let $w$ be an element of $\bar{\Omega}_{A} \vee$. Consider a generating mapping $\varphi: A \rightarrow S$ of a semigroup in $\mathrm{V}$. For every stationary point $p$ of $\mathfrak{L}(w)$, there is a clopen interval I containing $p$ such that, if $t$ is a transition between elements $q, r$ of $I$ with $q \leq r$, then $\varphi_{\mathrm{V}}(t)$ is a factor of the elements of $\varphi \vee\left(J_{p}\right)$.

Proof: Let $(u, v) \in p$. By Proposition 7.13 and its dual, there is in $\mathfrak{F}(w)$ an increasing sequence $\left(p_{n}\right)_{n}$ of step points converging to $(u, v)$ and a 
decreasing sequence $\left(p_{n}^{\prime}\right)_{n}$ of step points converging to $(u, v)$. Denote by $\tau_{n}$ the unique edge from $p_{n}$ to $(u, v)$, and by $\tau_{n}^{\prime}$ the unique edge from $(u, v)$ to $p_{n}^{\prime}$ (uniqueness of these edges is given by Proposition 7.6). Let $\varepsilon$ be a loop in $\mathcal{T}_{p}$ at vertex $(u, v)$. Then $\tau_{n} \varepsilon \tau_{n}^{\prime}$ converges to a loop $\tilde{\varepsilon}$ of $\mathcal{T}(w)$ at vertex $(u, v)$. Since $\varepsilon$ is a factor of $\tilde{\varepsilon}$, we must have $\Lambda(\tilde{\varepsilon}) \in J_{p}$ (thus we have $\tilde{\varepsilon}=\varepsilon$ by Corollary 9.4 , although we shall not use that fact). Therefore, since $\varphi_{\mathrm{A}}$ and the labeling mapping $\Lambda$ are continuous, there is $N$ such that $\varphi \vee\left(\Lambda\left(\tau_{n} \varepsilon \tau_{n}^{\prime}\right)\right) \in \varphi_{\vee}\left(J_{p}\right)$ for all $n \geq N$. Consider the clopen interval $I=\left[p_{N}, p_{N}^{\prime}\right]$. Let $q$ and $r$ be elements of $I$ such that $q \leq r$, and let $t$ be a transition from $q$ to $r$. Then $t$ is a factor of a transition from $p_{N}$ to $p_{N}^{\prime}$. But $\Lambda\left(\tau_{N} \varepsilon \tau_{N}^{\prime}\right)$ is the unique transition from $p_{N}$ to $p_{N}^{\prime}$, by Proposition 7.6. Hence, $\varphi \vee(t)$ is a factor of the elements of $\varphi \mathrm{v}\left(J_{p}\right)$.

Lemma 11.11. Let $\left(q_{n}\right)_{n}$ be a sequence of stationary points of $\mathfrak{L}(w)$ converging to $q$. For each $n$, let $u_{n}$ be an element of $J_{q_{n}}$. Suppose that $\left(u_{n}\right)_{n}$ converges to $u$. Then $u$ is J-above $J_{q}$.

Proof: The sequence of edges $\left(q_{n} \stackrel{u_{n}}{\rightarrow} q_{n}\right)_{n}$ of $\mathcal{T}(w)$ converges to $q \stackrel{u}{\rightarrow} q$. Since $u$ labels a transition from $q$ to itself, $u$ is $\mathcal{J}$-above $J_{q}$.

In Lemma 11.11 it is not true in general that $u \in J_{q}$. In Example 8.1, the stationary point $r$ such that $\left(a^{\omega} b a^{\omega}\right)^{\omega}$ belongs to $J_{r}$ is the limit of a sequence $\left(q_{n}\right)_{n}$ of stationary points such that $a^{\omega} \in J_{q_{n}}$, but $a^{\omega} \notin J_{r}$.

Definition 11.12 (Mapping $\Gamma$ ). Let $J_{S}$ be the set of regular $\mathcal{J}$-classes of $S$, and denote by $\Upsilon$ the mapping stat $(\mathfrak{L}(w)) \rightarrow J_{S}$ sending a stationary point $p$ to the $\mathcal{J}$-class containing $\varphi_{\mathrm{A}}\left(J_{p}\right)$. Denote by $\Gamma$ the mapping $\Upsilon \times$ $F_{g}: \operatorname{stat}(\mathfrak{L}(w)) \rightarrow J_{S} \times \operatorname{Im}\left(F_{g}\right)$ sending $p$ to $\left(\Upsilon(p), F_{g}(p)\right)$.

In the next lemma, we continue to assume that $\mathfrak{L}_{c}(w)$ is $g$-recognized by $(\varphi, s)$.

Lemma 11.13. Suppose that the semigroup $S$ is unambiguous. Let $X, Y$ be elements of $\mathcal{O}_{w}$ such that $X<Y$. Suppose that the restriction of $\Gamma$ to $\operatorname{stat}(\mathfrak{L}(w)) \cap[X, Y[$ is constant. Then $[X, Y[$ is a special bridge.

Proof: Let $p \in \operatorname{stat}(\mathfrak{L}(w)) \cap[X, Y[$. By Lemma 11.6, it suffices to prove that there is a special bridge containing $p$. Let $I$ be a clopen interval containing $p$ satisfying the properties described in Lemma 11.10, and let $\left[x_{0}, y_{0}\right]$ be the clopen interval $I \cap[X, Y$, which is indeed nonempty as it contains $p$. Note that $x_{0}$ and $y_{0}$ are step points. Let $X^{\prime}=\mathcal{O}_{w}\left(x_{0}\right)$ and $Y^{\prime}=\mathcal{O}_{w}\left(y_{0}\right)$. By the definition of $\mathcal{O}_{w}, x_{0} \geq X$ implies $X^{\prime} \geq X$, and $y_{0} \leq Y$ implies $Y^{\prime} \leq Y$, thus $\left[X^{\prime}, Y^{\prime}[\subseteq[X, Y[\right.$. Therefore, we have $p \in\left[X^{\prime}, Y^{\prime}\left[\right.\right.$ and $\left[\rho\left(X^{\prime}\right), \lambda\left(Y^{\prime}\right)\right] \subseteq I \cap[X, Y[$. 
Let $x, y$ be step points in $\left[X^{\prime}, Y^{\prime}[\right.$ such that $x<y$. We want to show that $x \rightarrow y$ is $g$-projected. If $x \prec \prec y$, then we apply Remark 11.3, so we suppose that is not the case, which implies $\rho(x) \leq \lambda(y)$. Note that $[\rho(x), \lambda(y)] \subseteq\left[\rho\left(X^{\prime}\right), \lambda\left(Y^{\prime}\right)\right]$. If $t$ is a $\mathcal{J}$-minimum transition from $\rho(x)$ to $\lambda(y)$ then $t \leq_{\mathcal{J}} J_{\rho(x)}$ and $t \leq_{\mathcal{J}} J_{\lambda(y)}$ by Lemma 11.7, thus $\varphi_{\mathrm{A}}(t) \leq_{\mathcal{J}} \Upsilon(\rho(x))$ and $\varphi_{\mathrm{A}}(t) \leq_{\mathcal{J}} \Upsilon(\lambda(y))$. Hence $\varphi_{\mathrm{A}}(t) \leq_{\mathfrak{J}} \Upsilon(p)$, because the restriction of $\Upsilon$ to $\operatorname{stat}(\mathfrak{L}(w)) \cap[X, Y[$ is constant. By the choice of $I$ (cf. Lemma 11.10), this implies $\varphi_{\mathrm{A}}(t) \in \Upsilon(p)$. Therefore, since the restriction of $\Gamma$ to stat $(\mathfrak{L}(w)) \cap[X, Y[$ is constant, we have shown that the pair $(\rho(x), \lambda(y))$ is a $\mathcal{J}$-bridge. By Remark 11.3, $[x, \rho(x)[$ and $] \lambda(y), y]$ are bridges, whence it follows from Proposition 11.9 that there are step points $x^{\prime} \in\left[x, \rho(x)\left[\right.\right.$ and $\left.\left.y^{\prime} \in\right] \lambda(y), y\right]$ such that $x^{\prime} \rightarrow y^{\prime}$ is $g$-projected. Therefore $x \rightarrow y$ is $g$-projected by Remarks 11.3 and 11.2. This concludes the proof that $\left[X^{\prime}, Y^{\prime}[\right.$ is a special bridge containing $p$.

Proposition 11.14. Suppose that the finite aperiodic semigroup $S$ is unambiguous. Then every stationary point $p$ of $\mathfrak{L}(w)$ is contained in some special bridge.

Proof: Endow $J_{S} \times \operatorname{Im}\left(F_{g}\right)$ with the following partial order:

$$
\left(J_{1}, P_{1}\right) \leq\left(J_{2}, P_{2}\right) \Longleftrightarrow\left(J_{2}<_{\mathfrak{g}} J_{1} \text { or }\left(J_{1}=J_{2} \text { and } P_{1} \subseteq P_{2}\right)\right) .
$$

For each $p \in \operatorname{stat}(\mathfrak{L}(w))$, let

$$
G(p)=\{\Gamma(q): q \in \operatorname{stat}(\mathfrak{L}(w)) \text { and } \Gamma(q)<\Gamma(p)\}
$$

We shall prove by induction on the cardinal of $G(p)$ that $p$ is contained in some special bridge.

We start with some preliminary remarks. Let $I=[\alpha, \beta]$ be a clopen interval containing $p$ satisfying the properties described in Lemmas 11.10 and 8.4(b). Let $X=\mathcal{O}_{w}(\alpha)$ and $Y=\mathcal{O}_{w}(\beta)$. Then the following holds:

$$
\operatorname{stat}(\mathfrak{L}(w)) \cap I=\operatorname{stat}(\mathfrak{L}(w)) \cap[X, Y[.
$$

Let $q \in \operatorname{stat}(\mathfrak{L}(w)) \cap[X, Y[$. Then we have $\Upsilon(p) \leq \mathcal{J} \Upsilon(q)$ by the choice of $I$ (cf. Lemma 11.10). It also follows from the choice of $I$ (cf. Lemma 8.4(b)) that $F_{g}(q) \subseteq F_{g}(p)$. Hence we have

$$
\Gamma(q) \leq \Gamma(p) \quad \text { for all } q \in \operatorname{stat}(\mathfrak{L}(w)) \cap[X, Y[
$$


Initial step. Suppose that $G(p)=\emptyset$. It follows from (11.3) that $\Gamma(q)=$ $\Gamma(p)$ for every $q \in \operatorname{stat}(\mathfrak{L}(w)) \cap[X, Y[$. Therefore, $[X, Y[$ is a special bridge, by Lemma 11.13 .

Inductive step. Suppose that for every stationary point $q$ such that $|G(q)|<|G(p)|$, there is a special bridge containing $q$. Consider the sets

$$
\begin{aligned}
M & =\{q \in \operatorname{stat}(\mathfrak{L}(w)) \cap[X, Y[: \Gamma(q)<\Gamma(p)\}, \\
N & =\{q \in \operatorname{stat}(\mathfrak{L}(w)) \cap[X, Y[: \Gamma(q)=\Gamma(p)\} .
\end{aligned}
$$

Note that $M \cup N=\operatorname{stat}(\mathfrak{L}(w)) \cap[X, Y$, by (11.3). Note also that, by the induction hypothesis, every element of $M$ is contained in some special bridge, as $\Gamma(q)<\Gamma(p)$ implies $G(q) \subsetneq G(p)$ with $\Gamma(q) \in G(p) \backslash G(q)$. Our goal is to apply Lemma 11.6 to the interval $[X, Y[$. So, it remains to show that every element of $N$ is contained in some special bridge. For that purpose, we prove the following lemma.

Lemma 11.15. The set $N$ is closed.

Proof: Consider a sequence $\left(q_{n}\right)_{n}$ of elements of $N$ converging to $q$. Since $[X, Y[$ and $\operatorname{stat}(\mathfrak{L}(w))$ are closed (the latter in view of Proposition 7.5), we only need to prove that $\Gamma(q)=\Gamma(p)$.

By Lemma 8.4(b), we have $F_{g}\left(q_{n}\right) \subseteq F_{g}(q)$ for sufficiently large $n$. By the definition of $N$, we have $F_{g}\left(q_{n}\right)=F_{g}(p)$ for all $n$, whence $F_{g}(p) \subseteq$ $F_{g}(q)$. On the other hand, since $q \in[X, Y[$ and thus $q \in I$, by the choice of $I$ (cf. Lemma 8.4(b)) we have $F_{g}(q) \subseteq F_{g}(p)$. This concludes the proof of the equality $F_{g}(q)=F_{g}(p)$.

By Lemma 11.11, we have $\Upsilon(q) \leq_{\mathfrak{J}} \Upsilon\left(q_{n}\right)$ for some sufficiently large $n$. By the definition of $N$, we have $\Upsilon(p)=\Upsilon\left(q_{n}\right)$ for all $n$, whence $\Upsilon(q) \leq_{\mathcal{J}}$ $\Upsilon(p)$. On the other hand, since $q \in\left[X, Y\right.$ [ we have $\Upsilon(p) \leq_{\text {J }} \Upsilon(q)$ by (11.3). Therefore we have $\Gamma(p)=\Gamma(q)$.

Let us proceed with the proof of Proposition 11.14. Suppose that $q \in N$. Let $I^{\prime}$ be a clopen interval containing $q$, and contained in $[X, Y$, satisfying the properties described in Lemmas 11.10 and 8.4(b). In a similar way as we have built $X, Y$ from $I$, we may build from $I^{\prime}$ elements $X^{\prime}, Y^{\prime} \in \mathcal{O}_{w}$ such that $q \in\left[X^{\prime}, Y^{\prime}\left[\right.\right.$ and $\operatorname{stat}(\mathfrak{L}(w)) \cap\left[X^{\prime}, Y^{\prime}\left[\subseteq I^{\prime}\right.\right.$.

Let $x$ and $y$ be two step points of $\left[X^{\prime}, Y^{\prime}[\right.$ such that $x<y$. We want to prove that the edge $x \rightarrow y$ is $g$-projected. By Remark 11.3, we may as well assume that $x$ and $y$ are not $\approx$-equivalent.

Suppose $N \cap[x, y]=\emptyset$. Then every element of the nonempty intersection $\operatorname{stat}(\mathfrak{L}(w)) \cap[x, y$ [ belongs to $M$ and is therefore contained in 
a special bridge, as observed earlier. Hence $\left[\mathcal{O}_{w}(x), \mathcal{O}_{w}(y)[\right.$ is a special bridge by Lemma 11.6. It follows that the edge $x \rightarrow y$ is $g$-projected (cf. Remarks 11.3 and 11.2).

Suppose that $N \cap[x, y] \neq \emptyset$. Then $N \cap[x, y]$ has a minimum $r_{x}$ and a maximum $r_{y}$, because $N \cap[x, y]$ is closed by Lemma 11.15.

Let $t$ be a $\mathcal{J}$-minimum transition from $r_{x}$ to $r_{y}$. Then we have $t \leq \mathcal{J} J_{r_{x}}$ by Lemma 11.7, thus $\varphi_{\mathrm{A}}(t) \leq_{\mathcal{J}} \Upsilon\left(r_{x}\right)$. On the other hand, $r_{x}, r_{y} \in I^{\prime}$, and so, by the choice of $I^{\prime}$ (cf. Lemma 11.10), we know that $\varphi_{\mathrm{A}}(t) \geq_{\mathrm{f}}$ $\Upsilon(q)$. Since by the definition of $N$, the equalities $\Upsilon\left(r_{x}\right)=\Upsilon\left(r_{y}\right)=\Upsilon(q)$ hold, we obtain $\varphi_{\mathrm{A}}(t) \in \Upsilon(q)$. Also by the definition of $N$, we conclude that $F_{g}\left(r_{x}\right)=F_{g}\left(r_{y}\right)$. This shows that $\left(r_{x}, r_{y}\right)$ is a $\mathcal{J}$-bridge.

We claim that $\left[x, r_{x}\right.$ [ is a bridge. Let $x_{1}$ and $x_{2}$ be step points such that $x<x_{1} \leq x_{2}<r_{x}$. We want to show that $x_{1} \rightarrow x_{2}$ is $g$-projected, for which we may as well assume that $x_{1}$ and $x_{2}$ are not $\approx$-equivalent. Recall that $M \cup N=\operatorname{stat}(\mathfrak{L}(w)) \cap[X, Y[$ by (11.3), and so, by the definition of $r_{x}$, the set stat $(\mathfrak{L}(w)) \cap\left[\mathcal{J}_{w}\left(x_{1}\right), \mathcal{O}_{w}\left(x_{2}\right)\right.$ [ is contained in $M$. But we already observed that every element of $M$ is contained in some special bridge. Hence, $\left[\mathcal{O}_{w}\left(x_{1}\right), \mathcal{O}_{w}\left(x_{2}\right)\right.$ [ is a special bridge by Lemma 11.6, and so $x_{1} \rightarrow x_{2}$ is $g$-projected (cf. Remarks 11.3 and 11.2), thus proving the claim. Similarly, $\left.] r_{y}, y\right]$ is a bridge.

By Proposition 11.9, there are step points $x_{0}$ and $y_{0}$ satisfying $x<$ $x_{0}<r_{x}$ and $r_{y}<y_{0}<y$ such that $x_{0} \rightarrow y_{0}$ is $g$-projected. Again by the definition of $r_{x}$ and by (11.3), every element of $\operatorname{stat}(\mathfrak{L}(w)) \cap$ $\left[\mathcal{O}_{w}(x), \mathcal{O}_{w}\left(x_{0}\right)\right.$ [ belongs to $M$, and so $\left[\mathcal{O}_{w}(x), \mathcal{O}_{w}\left(x_{0}\right)[\right.$ is a special bridge by Lemma 11.6. Hence, the edge $x \rightarrow x_{0}$ is $g$-projected. Similarly, $y_{0} \rightarrow$ $y$ is $g$-projected. It follows that $x \rightarrow y$ is $g$-projected by Remark 11.2.

We showed in all cases that $x \rightarrow y$ is $g$-projected. Hence, $\left[X^{\prime}, Y^{\prime}\right.$ [ is a special bridge containing $q$.

We have established that every element of $\operatorname{stat}(\mathfrak{L}(w)) \cap[X, Y[$ is contained in some special bridge. From Lemma 11.6, we then deduce that $[X, Y[$ is a special bridge containing $p$. This concludes the inductive step in our proof, thereby showing that the proposition holds.

Theorem 11.16. Let $w$ be an element of $\bar{\Omega}_{A} \mathrm{~A}$. Let $\varphi: A \rightarrow S$ be a generating mapping of a finite aperiodic unambiguous semigroup $S$. Suppose that $\mathfrak{L}_{c}(w)$ is g-recognized by $(\varphi, s)$. Then $\mathfrak{L}(w)$ is a bridge.

Proof: Clearly, if $w \in A^{+}$then $\mathfrak{L}(w)$ is a bridge by Remark 11.3. Suppose that $w \in \bar{\Omega}_{A} \mathrm{~A} \backslash A^{+}$. By Proposition 11.14, for each stationary point $p$ of $\mathfrak{L}(w)$, there is a special bridge $\left[X_{p}, Y_{p}\right.$ [ containing it. The union of the nonempty family $\left(\left[X_{p}, Y_{p}[)_{p \in \operatorname{stat}(\mathfrak{L}(w))}\right.\right.$ is $\mathfrak{L}(w) \backslash\{(w, 1)\}$, therefore $\mathfrak{L}(w)$ is a bridge, in view of Lemma 11.5. 
Conclusion of the proof of Theorem 8.6: The direct implication in Theorem 8.6 follows directly from Proposition 8.5.

Conversely, suppose that $\mathfrak{L}(w)$ is $g$-recognized by $(\varphi, s)$, for some $g$. Then $(1, w) \stackrel{w}{\longrightarrow}(w, 1)$ is $g$-projected to $g(1, w) \stackrel{\varphi_{A}(w)}{\longrightarrow} g(w, 1)$ by Theorem 11.16. But $g(1, w)=(1, s)$ and $g(w, 1)=(s, 1)$. It follows that $s=\varphi_{\mathrm{A}}(w)$.

\section{The effect of multiplication on the quasi-order}

In this section, under suitable conditions, we relate $\mathfrak{F}(u v)$ on one hand, with $\mathfrak{F}(u)$ and $\mathfrak{F}(v)$ on the other hand.

The quasi-orders considered in this section are all total, as they stem from the quasi-order of 2-factorizations of equidivisible semigroups. We want to compare different intervals of quasi-ordered sets of 2-factorizations. This leads us to introduce the following definitions. Let $(P, \leq)$ and $(Q, \leq)$ be two quasi-ordered sets, and let $\varphi$ be a function from $P$ to $Q$. Recall that $\varphi$ is monotone if $p \leq q$ implies $\varphi(p) \leq \varphi(q)$, for every $p, q \in P$. Suppose moreover that the quasi-order on $P$ is total. Then we say that $\varphi$ is a quasi-isomorphism if $\varphi$ is a surjective monotone mapping such that, for all $p, q \in P$, we have $p<q \Rightarrow \varphi(p)<\varphi(q)$. Because the quasi-order on $P$ is total, we have $\varphi(p)<\varphi(q) \Rightarrow p<q$ and $\varphi(p) \sim \varphi(q) \Rightarrow p \sim q$, for all $p, q \in P$. Therefore, $\varphi$ induces the isomorphism of linearly ordered sets $\tilde{\varphi}: P / \sim \rightarrow Q / \sim \operatorname{sending} p / \sim$ to $\varphi(p) / \sim$. In particular, the quasi-order on $Q$ is also total.

Let $I_{u}$ be an interval of $\mathfrak{L}(u)$ and $I_{v}$ an interval of $\mathfrak{L}(v)$, for some $u, v$ in a compact semigroup $S$. A mapping $\theta: I_{u} \rightarrow I_{v}$ is said to be $J$-preserving if $J_{p}=J_{\theta(p)}$ for every $p \in I_{u}$.

Proposition 12.1. Consider an equidivisible profinite semigroup $S$ which is finitely cancelable, and take $w \in S$. Let $(u, v) \in \mathfrak{F}(w)$. For $p=(u, v) / \sim$, let e be the unique idempotent of $J_{p}$ stabilizing $(u, v)$. Then the following mappings are quasi-isomorphisms of intervals of the respective totally quasi-ordered sets $\mathfrak{F}(w), \mathfrak{F}(u)$, and $\mathfrak{F}(v)$ :

$$
\begin{aligned}
\lambda_{(u, v)}:[(1, u),(u, e)] & \rightarrow[(1, w),(u, v)] \\
(x, y) & \mapsto(x, y v), \\
\rho_{(u, v)}:[(e, v),(v, 1)] & \rightarrow[(u, v),(w, 1)] \\
(x, y) & \mapsto(u x, y) .
\end{aligned}
$$

Moreover, the induced isomorphisms $\tilde{\lambda}_{(u, v)}$ and $\tilde{\rho}_{(u, v)}$ are J-preserving. 
Before proceeding with the proof of Proposition 12.1, let us recall that the uniqueness of $e$ mentioned in its statement is guaranteed by Lemma 5.3. The next technical lemma will be used in the proof of Proposition 12.1.

Lemma 12.2. Let $S$ be an equidivisible compact semigroup, and let $x, y, z \in S$. If $x y=x y z$, then there exists some idempotent $e \in S^{I}$ such that $y z^{\omega}=e y$ and $x e=x$. Dually, if $y z=x y z$, then there exists some idempotent $e \in S^{I}$ such that $x^{\omega} y=$ ye and $e z=z$.

Proof: We deal only with the case $x y=x y z$, as the other case is dual. Since $S$ is equidivisible, the pairs $(x, y)$ and $(x, y z)$ are comparable elements of the quasi-ordered set $\mathfrak{F}(x y)$. If $(x, y z) \leq(x, y)$, then there exists $t \in S^{I}$ such that $x=x t$ and $y z=t y$, and so $y z^{k}=t^{k} y$ for every $k \geq 1$, whence $y z^{\omega}=t^{\omega} y$ and we choose $e=t^{\omega}$. Otherwise, $(x, y)<(x, y z)$, and so there exists $u \in S$ such that $x=x u$ and $y=u y z$, whence $x=x u^{\omega}$ and $y=u^{\omega} y z^{\omega}$; since $y z^{\omega}=y=u^{\omega} y$, we may choose $e=u^{\omega}$.

Proof of Proposition 12.1: By symmetry, it suffices to consider the mapping $\lambda=\lambda_{(u, v)}$. By Remark 4.6, and since $e v=v$, the mapping $\lambda$ indeed takes its values in the interval $[(1, w),(u, v)]$ and it is monotone.

Let $(x, z) \in \mathfrak{F}(w)$ be such that $(x, z) \leq(u, v)$. Then there is $t \in S^{I}$ such that $x t=u$ and $z=t v$. And, since $u=u e$, we deduce that $(x, t e) \stackrel{t}{\rightarrow}(u, e)$ is and edge of $\mathcal{T}(u)$, whence $(x, t e)$ belongs to the interval $[(1, u),(u, e)]$. As $\lambda(x, t e)=(x, t e v)=(x, z)$, we conclude that $\lambda$ is surjective.

To prove that $\lambda$ is a quasi-isomorphism, it remains to show that if $\left(x_{1}, y_{1}\right),\left(x_{2}, y_{2}\right)$ are elements of $[(1, u),(u, e)]$, then

$$
\left(x_{1}, y_{1}\right)<\left(x_{2}, y_{2}\right) \Longrightarrow\left(x_{1}, y_{1} v\right)<\left(x_{2}, y_{2} v\right) .
$$

Reasoning by reductio ad absurdum, suppose that the implication fails, that is, that $\left(x_{1}, y_{1}\right)<\left(x_{2}, y_{2}\right)$ and $\left(x_{2}, y_{2} v\right) \leq\left(x_{1}, y_{1} v\right)$. We may then consider $s, t \in S^{I}$ such that $x_{1} t=x_{2}, y_{1}=t y_{2}, x_{2} s=x_{1}$, and $y_{2} v=s y_{1} v$. The latter equality can be written as $y_{2} v=s t \cdot y_{2} v$, and applying the second case of Lemma 12.2 to it, we conclude that there exists an idempotent $f \in S^{I}$ such that $(s t)^{\omega} y_{2}=y_{2} f$ and $f v=v$. The calculations

$$
x_{2} \cdot(s t)^{\omega-1} s=x_{1}(t s)^{\omega}=x_{1} \text { and }(s t)^{\omega-1} s \cdot y_{1}=(s t)^{\omega} y_{2}=y_{2} f
$$

show that $x_{2} \cdot y_{2} f=x_{1} y_{1}=u$ and

$$
\left(x_{2}, y_{2} f\right) \leq\left(x_{1}, y_{1}\right)
$$


in $[(1, u),(u, e)]$. Since $\left(x_{1}, y_{1}\right)<\left(x_{2}, y_{2}\right)$, we reach a contradiction provided we prove that $y_{2} f=y_{2}$.

Recall that $v=f v$, and note that $u=x_{2} y_{2} f$ implies $u=u f$. Hence, as $(u, v) \stackrel{e}{\rightarrow}(u, v)$ belongs to the ideal $\mathcal{K}_{p}$, we conclude that $(u, v) \stackrel{\text { efe }}{\longrightarrow}$ $(u, v)$ also belongs to $\mathcal{K}_{p}$. From Corollary 9.4, we get efe $=e$. On the other hand, since $\left(x_{2}, y_{2} f\right) \leq(u, e)($ cf. $(12.2))$ and $\left(x_{2}, y_{2}\right) \leq(u, e)$, we have $y_{2} f e=y_{2} f$ and $y_{2} e=y_{2}$. Hence, $y_{2} f=y_{2} f e=y_{2} e f e=$ $y_{2} e=y_{2}$, and so we reach the desired contradiction. The contradiction was originated by the assumption that the implication (12.1) fails in the interval $[(1, u),(u, e)]$. Hence, the implication holds, which concludes the proof that $\lambda$ is a quasi-isomorphism.

It remains to show that, for $(x, y) \in[(1, u),(u, e)]$, we have $J_{q}=J_{\tilde{\lambda}(q)}$, where $q=(x, y) / \sim$. Let $\varepsilon \in S^{I}$ be an idempotent. Observe that if $\varepsilon$ stabilizes $(x, y)$ then it also stabilizes $(x, y v)$, which shows that $J_{q}$ lies J-above $J_{\tilde{\lambda}(q)}$.

Conversely, suppose that $\varepsilon$ stabilizes $(x, y v)$. As $\varepsilon y v=y v$, it follows from Lemma 12.2 that there is some idempotent $f \in S^{I}$ with $\varepsilon y=y f$ and $f v=v$.

We claim that $(x, y f) \leq(u, e)$. Suppose on the contrary that

$$
(u, e)<(x, y f) \text {. }
$$

Then $e \leq_{\mathcal{L}} y f$, and so in particular we have $e=e f$. Similarly, from

$$
(x, y) \leq(u, e)
$$

we get $y=y e$. Therefore, $y f=y e f=y e=y$, yielding a contradiction between (12.3) and (12.4). This shows the claim that $(x, y f) \leq(u, e)$.

We are now assured that $(x, y f)$ belongs to the domain of $\lambda$. From $v=$ $f v$, we get $\lambda(x, y)=(x, y v)=\lambda(x, y f)$. We have already proved that $\lambda$ is a quasi-isomorphism, and so we conclude that $(x, y) \sim(x, y f)$. On the other hand, $\varepsilon$ clearly stabilizes $(x, y f)=(x, \varepsilon y)$. Hence, $J_{\tilde{\lambda}(q)}$ lies $\mathcal{J}$-above $J_{q}$, which proves that the two $\mathcal{J}$-classes coincide.

Remark 12.3. Notice that in Proposition 12.1, in the special case where $(u, v)$ is a step point, we have $e=1$ and so the domains of $\lambda_{(u, v)}$ and $\rho_{(u, v)}$ are, respectively, $\mathfrak{F}(u)$ and $\mathfrak{F}(v)$.

Remark 12.4. Suppose that in Proposition 12.1 we have $e \neq 1$. Let $q$ be the stationary point $(e, e) / \sim$ of $\mathfrak{L}(e)$. Since $e$ stabilizes $(e, e)$ and $J_{q}$ is $\mathcal{J}$-above $e$, we have $e \in J_{q}$. Therefore, applying Proposition 12.1, we may consider the quasi-isomorphisms $\lambda_{(e, v)}:[(1, e),(e, e)] \rightarrow[(1, v),(e, v)]$ and $\rho_{(u, e)}:[(e, e),(e, 1)] \rightarrow[(u, e),(u, 1)]$. 
The diagram in Figure 3 may facilitate the understanding of the applications of Proposition 12.1 in the case in which $(u, v)$ is a stationary point.

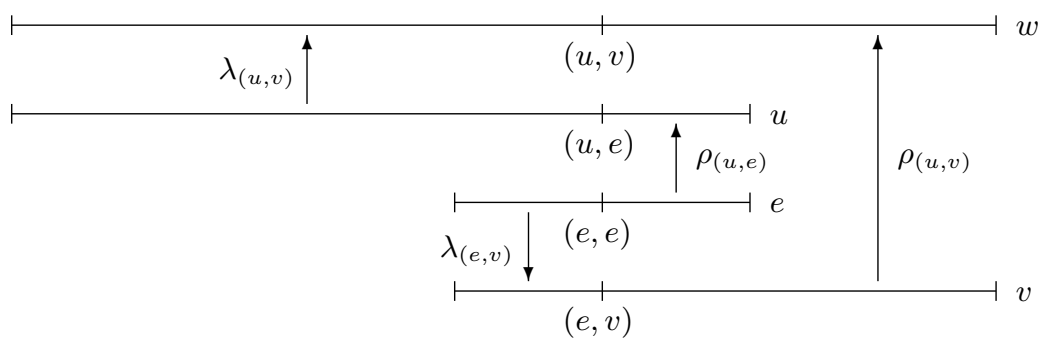

FIGURE 3. Quasi-isomorphisms associated with a stationary point $(u, v)$.

The arrows indicate quasi-isomorphisms between various intervals of the quasi-ordered sets $\mathfrak{F}(w), \mathfrak{F}(u), \mathfrak{F}(v)$, and $\mathfrak{F}(e)$. Those quasi-isomorphisms induce isomorphisms between the corresponding intervals of the linearly ordered sets $\mathfrak{L}(w), \mathfrak{L}(u), \mathfrak{L}(v)$, and $\mathfrak{L}(e)$. The picture is perhaps clearer if interpreted in this context, in which case, the points $(u, v),(u, e),(e, v)$, and $(e, e)$ should be replaced by their respective $\sim$-classes.

Let $S$ be an equidivisible profinite semigroup $S$ which is finitely cancelable, and let $w \in S$. We endow every ordered subset $Q$ of $\mathfrak{L}(w)$ with the following labeling $l$ : for a step point $p=(u, v)$ of $\mathfrak{L}(w)$ belonging to $Q$, let $l(p)=\mathrm{i}(v)$ (and so if $Q=\mathfrak{L}(w)$ then the labeling on step points is the one defining the cluster word $\left.\mathfrak{L}_{c}(w)\right)$; for a stationary point $p$ of $\mathfrak{L}(w)$ belonging to $Q$, let $l(p)=J_{p}$. The resulting labeled ordered set is denoted $Q_{l}$. In the next result, $P_{l}+Q_{l}$ denotes the labeled ordered set with underlying ordered set $P+Q$ and labeling whose restriction to $P$ and $Q$ is respectively the labeling of $P_{l}$ and $Q_{l}$. The symbol $\cong$ stands for isomorphism of labeled ordered sets.

Proposition 12.5. Let $S$ be an equidivisible profinite semigroup $S$ which is finitely cancelable. Take $w \in S$. Let $u, v \in S$ be such that $w=u v$. If

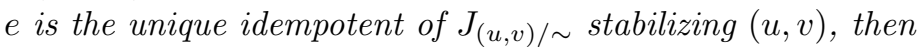

$$
\mathfrak{L}(w)_{l} \cong\left[(1, u) / \sim,(u, e) / \sim\left[_{l}+[(e, v) / \sim,(v, 1) / \sim]_{l} .\right.\right.
$$

In particular, if $(u, v)$ is a step point, then $\mathfrak{L}(w)_{l} \cong(\mathfrak{L}(u) \backslash\{(u, 1)\})_{l}+$ $\mathfrak{L}(v)_{l}$.

Proof: Consider the quasi-isomorphism $\lambda_{(u, v)}$ and respective isomorphism $\tilde{\lambda}_{(u, v)}$ as in Proposition 12.1. Then, the pair $(x, y) \in \mathfrak{F}(u)$ is a step 
point of the interval $\left[(1, u),(u, e)\left[\right.\right.$ if and only if its image $\lambda_{(u, v)}(x, y)=$ $(x, y v)$ is a step point of $[(1, w),(u, v)[$, and $\mathrm{i}(y)=\mathrm{i}(y v)$. This fact, together with $\tilde{\lambda}_{(u, v)}$ being $J$-preserving, enables us to conclude that

$$
\left[(1, u) / \sim,(u, e) / \sim\left[_ { l } \cong \left[(1, w) / \sim,(u, v) / \sim\left[_{l} .\right.\right.\right.\right.
$$

Similarly, we obtain

$$
[(e, v) / \sim,(v, 1) / \sim]_{l} \cong[(u, v) / \sim,(w, 1) / \sim]_{l} .
$$

Since we clearly have

$$
\mathfrak{L}(w)_{l}=[(1, w) / \sim,(u, v) / \sim)\left[_{l}+[(u, v) / \sim,(w, 1) / \sim]_{l},\right.
$$

this concludes the proof.

\section{The image of the representation $w \mapsto \mathfrak{L}_{c}(w)$ in the aperiodic case}

Consider a cluster word $(L, \ell)$ over $A$. Let $\varphi: A \rightarrow S$ be a generating mapping of a semigroup $S$. Let $s \in S$ and $g: \operatorname{step}(L) \rightarrow \mathfrak{F}(s)$ be such that $(L, \ell)$ is $g$-recognized by $(\varphi, s)$. We say that $g$ is a $(\varphi, s)$-recognizer of $(L, \ell)$.

Lemma 13.1. Let $\varphi: A \rightarrow S$ be a generating mapping of a finite semigroup $S$, and let $\pi: S \rightarrow T$ be an onto homomorphism of semigroups. Suppose that $(L, \ell)$ is recognized by $(\varphi, s)$. Then $(L, \ell)$ is recognized by $(\pi \circ \varphi, \pi(s))$.

Proof: Let $g: \operatorname{step}(L) \rightarrow \mathfrak{F}(s)$ be a $(\varphi, s)$-recognizer of $(L, \ell)$. For each $p \in \operatorname{step}(L)$, let $g(p)=\left(u_{p}, v_{p}\right)$. Consider the mapping $h: \operatorname{step}(L) \rightarrow$ $\mathfrak{F}(\pi(s))$ defined by $h(p)=\left(\pi\left(u_{p}\right), \pi\left(v_{p}\right)\right)$. We claim that $(L, \ell)$ is $h$-recognized by $(\pi \circ \varphi, \pi(s))$. The conditions (R.1)-(R.3) in Definition 8.2 for $h$-recognition by $(\pi \circ \varphi, \pi(s))$ are clearly satisfied. It remains to show that condition (R.4) holds. Let $p$ be a stationary point of $(L, \ell)$. Take an element $q$ of $\mathfrak{F}(\pi(s))$ such that $h^{-1}(q)$ is left cofinal at $p$. Consider the set

$$
X=\{(u, v) \in \mathfrak{F}(s):(\pi(u), \pi(v))=q\} .
$$

Then we have $h^{-1}(q)=g^{-1}(X)=\bigcup_{x \in X} g^{-1}(x)$. Since $h^{-1}(q)$ is left cofinal at $p$ and $X$ is finite, there is at least one element $x_{0}$ of $X$ such that $g^{-1}\left(x_{0}\right)$ is left cofinal at $p$. But then $g^{-1}\left(x_{0}\right)$ is also right cofinal at $p$, because $(L, \ell)$ is $g$-recognized by $(\varphi, s)$. Therefore, $h^{-1}(q)$ is right cofinal at $p$. Symmetrically, if $h^{-1}(q)$ is right cofinal at $p$, then it is left cofinal at $p$. This concludes the proof. 
For a cluster word $(L, \ell)$ over $A$, if $p$ and $q$ are step points of $L$ such that $p \leq q$, then $([p, q], \ell)$ is the cluster word obtained from $(L, \ell)$ by restricting $\ell$ to $[p, q[$, and letting $\ell(q)=1$.

We wish to study cluster words $(L, \ell)$ satisfying the following conditions:

(W.1) For every finite aperiodic unambiguous $A$-generated semigroup $S$, and every generating mapping $\varphi: A \rightarrow S$, there is a unique $s \in S$ such that $(L, \ell)$ is recognized by $(\varphi, s)$.

(W.2) If $p$ and $q$ are step points of $L$ such that $p<q$, then $([p, q], \ell)$ satisfies (W.1).

(W.3) Consider an arbitrary finite aperiodic unambiguous $A$-generated semigroup $S$ and a generating mapping $\varphi: A \rightarrow S$. Let $s$ be such that $(L, \ell)$ if recognized by $(\varphi, s)$. Take a $(\varphi, s)$-recognizer $g$. Suppose $p$ and $q$ are step points such that $p<q$. If $t \in S$ is such that $(\varphi, t)$ recognizes $([p, q], \ell)$, then $g(p) \stackrel{t}{\rightarrow} g(q)$ is an edge of $\mathcal{T}(s)$.

Remark 13.2. In the setting of condition (W.3), there is only one such $(\varphi, s)$-recognizer, assuming that (W.1) and (W.2) also hold. Indeed, if $g$ is a $(\varphi, s)$-recognizer, and $p$ is a step point of $L$, and if $s_{1}$ and $s_{2}$ are (the unique) elements of $S$ such that $([\min L, p], \ell)$ and $([p, \max L], \ell)$ are respectively recognized by $\left(\varphi, s_{1}\right)$ and $\left(\varphi, s_{2}\right)$, then $g(\min L) \stackrel{s_{1}}{\rightarrow} g(p)$ and $g(p) \stackrel{s_{2}}{\longrightarrow} g(\max L)$ are edges of $\mathcal{T}(s)$, and so $g(p)=\left(s_{1}, s_{2}\right)$.

Finally we consider a fourth condition, assuming (W.1)-(W.3) hold:

(W.4) For every step point $p$ of $L$, there is a finite aperiodic unambiguous semigroup $S$ and a generating mapping $\varphi: A \rightarrow S$ such that, for the unique $(\varphi, s)$-recognizer $g$ of $(L, \ell)$, there are no elements of $S$ that stabilize $g(p)$ in $\mathcal{T}(S)$.

A cluster word satisfying conditions (W.1)-(W.4) is called a worthy cluster word.

Theorem 13.3. A cluster word $(L, \ell)$ over $A$ is isomorphic to a cluster word of the form $\mathfrak{L}_{c}(w), w \in \bar{\Omega}_{A} \mathrm{~A}$, if and only if it is a worthy cluster word.

Proof: Let $w \in \bar{\Omega}_{A}$ A. By Proposition 8.5 and Theorem 11.16, the cluster word $\mathfrak{L}_{c}(w)$ satisfies condition (W.1).

Take two step points $p$ and $q$ of $\mathfrak{L}_{c}(w)$ such that $p<q$. Let $t \in \bar{\Omega}_{A} \mathrm{~A}$ be the unique transition from $p$ to $q$. Applying twice Proposition 12.1, we conclude that $([p, q], \ell)$ is isomorphic with $\mathfrak{L}_{c}(t)$. Therefore, by the preceding paragraph, $([p, q], \ell)$ satisfies condition (W.1). 
By the previous paragraph and by Theorem 11.16, the cluster word $\mathfrak{L}_{c}(w)$ satisfies condition (W.3).

Suppose condition (W.4) does not hold for $\mathfrak{L}_{c}(w)$. Then, there is a step point $p$ such that, for every finite aperiodic unambiguous $A$-generated semigroup $S$ and every generating mapping $\varphi: A \rightarrow S$, the vertex $g(p)$ of $\mathcal{T}(s)$ is stabilized by some element of $S$. Let $p=(u, v)$. We then have $g(p)=\left(\varphi_{\mathrm{A}}(u), \varphi_{\mathrm{A}}(v)\right)$ (cf. Theorem 11.16). By a standard compactness argument, this implies that $(u, v)$ is stabilized by some element of $\bar{\Omega}_{A} \mathrm{~A}$. In view of Proposition 7.5, this is impossible since $p$ is a step point.

Conversely, suppose that $(L, \ell)$ is a worthy cluster word over $A$. Let $\left(\pi_{i}\right)_{i \in I}$ be an inverse system of continuous homomorphisms $\pi_{i}: \bar{\Omega}_{A} \mathrm{~A} \rightarrow$ $S_{i}$ onto finite aperiodic unambiguous $A$-generated semigroups, with connecting homomorphisms $\pi_{j, i}: S_{j} \rightarrow S_{i}$, such that $\bar{\Omega}_{A} \mathrm{~A}=\lim _{i \in I} S_{i}$. According to condition (W.1), for each $i \in I$, we may consider the unique element $s_{i}$ of $S_{i}$ such that $(L, \ell)$ is recognized by $\left(\pi_{i}, s_{i}\right)$. Applying Lemma 13.1, we then conclude that $\pi_{j, i}\left(s_{j}\right)=s_{i}$, whenever $i, j \in I$ are such that $i \leq j$. Hence, we may consider the unique element $w_{L}$ of $\bar{\Omega}_{A} \mathrm{~A}$ such that

$$
\pi_{i}\left(w_{L}\right)=s_{i}
$$

for every $i \in I$.

Consider the mapping $\lambda: \operatorname{step}(L) \rightarrow \operatorname{step}\left(\mathfrak{L}\left(w_{L}\right)\right)$ defined by

$$
\lambda(p)=\left(w_{[\min L, p]}, w_{[p, \max L]}\right) .
$$

Note that condition (W.2) ensures that $w_{[\min L, p]}$ and $w_{[p, \max L]}$ are well defined. We claim that $\lambda$ is an isomorphism between the cluster words $(L, \ell)$ and $\mathfrak{L}_{c}\left(w_{L}\right)$. In the process of proving this we show that $\left(w_{[\min L, p]}, w_{[p, \max L]}\right)$ is indeed a step point of $\mathfrak{F}\left(w_{L}\right)$ (and thus of $\mathfrak{L}\left(w_{L}\right)$ ).

We begin by observing that formula (13.1) generalizes to every generating mapping $\varphi: A \rightarrow S$ of a finite aperiodic unambiguous $A$-generated semigroup. Indeed, take $s \in S$ such that $(L, \ell)$ is recognized by $(\varphi, s)$. There is some $i \in I$ for which there is an onto homomorphism $\rho: S_{i} \rightarrow S$ satisfying $\varphi_{\mathrm{A}}=\rho \circ \pi_{i}$. By Lemma 13.1 and since $(L, \ell)$ satisfies (W.1), we know that $\rho\left(s_{i}\right)=s$. Therefore, we have

$$
\varphi_{\mathrm{A}}\left(w_{L}\right)=s
$$


For such a pair $(\varphi, s)$, let $g_{\varphi}: \operatorname{step}(L) \rightarrow \mathfrak{F}(s)$ be the unique $(\varphi, s)$-recognizer of $(L, \ell)$. If $p$ is a step point of $L$, then applying formula (13.2) to $[\min L, p]$ and to $[p, \max L]$, and taking into account Remark 13.2 , we conclude that

$$
g_{\varphi}(p)=\left(\varphi_{\mathrm{A}}\left(w_{[\min L, p]}\right), \varphi_{\mathrm{A}}\left(w_{[p, \max L]}\right)\right) .
$$

In particular, we have

$$
\varphi_{\mathrm{A}}\left(w_{[\min L, p]} w_{[p, \max L]}\right)=s=\varphi_{\mathrm{A}}\left(w_{L}\right) .
$$

Because $\varphi$ was arbitrarily chosen among generating mappings of finite aperiodic unambiguous $A$-generated semigroups, this shows that the pair $\lambda(p)=\left(w_{[\min L, p]}, w_{[p, \max L]}\right)$ indeed belongs to $\mathfrak{F}\left(w_{L}\right)$.

Consider step points $q$ and $r$ of $L$ such that $q \prec r$. Let $a=\ell(q)$. Take a generating mapping $\varphi: A \rightarrow S$ of a finite aperiodic unambiguous semigroup $S$ and $s \in S$ such that $(\varphi, s)$ recognizes $(L, \ell)$. By the definition of $(\varphi, s)$-recognizer, we can consider in $\mathcal{T}(s)$ the edge $g_{\varphi}(q) \stackrel{\varphi(a)}{\longrightarrow} g_{\varphi}(r)$. In view of formula (13.3), applied to $q$ and $r$, we then have

$\varphi_{\mathrm{A}}\left(w_{[\min L, q]} a\right)=\varphi_{\mathrm{A}}\left(w_{[\min L, r]}\right) \quad$ and $\quad \varphi_{\mathrm{A}}\left(w_{[q, \max L]}\right)=\varphi_{\mathrm{A}}\left(a w_{[r, \max L]}\right)$.

Since $\varphi$ was arbitrarily chosen among generating mappings of finite aperiodic unambiguous $A$-generated semigroups, we conclude that

$$
w_{[\min L, q]} a=w_{[\min L, r]} \quad \text { and } \quad w_{[q, \max L]}=a w_{[r, \max L]},
$$

that is, $\lambda(q) \stackrel{a}{\rightarrow} \lambda(r)$ is an edge of $\mathcal{T}\left(w_{L}\right)$. By Proposition 6.7 , we have either $\lambda(q) \sim \lambda(r)$ or $\lambda(q) \prec \lambda(r)$.

If $\lambda(q) \sim \lambda(r)$, then there is $z \in a\left(\bar{\Omega}_{A} \mathrm{~A}\right)^{I}$ such that $\lambda(q) \stackrel{z}{\rightarrow} \lambda(q)$ is an edge of $\mathcal{T}\left(w_{L}\right)$. Therefore, in view of formula (13.3), we conclude that $\varphi(z)$ labels a loop of $\mathcal{T}(s)$ rooted at $g_{\varphi}(q)$. This contradicts the assumption that condition (W.4) holds.

We then conclude that, for step points $q, r$ of $L$, we have

$$
q \prec r \Longrightarrow \lambda(q) \prec \lambda(r) \text {. }
$$

We also showed that $\ell(\lambda(q))=\ell(q)$, thus establishing that the mapping $\lambda: \operatorname{step}(L) \rightarrow \operatorname{step}\left(\mathfrak{L}\left(w_{L}\right)\right)$ has a well-defined codomain and that it preserves labels.

Notice that formula (13.3) can now be seen as follows: for the $(\varphi, s)$ recognizer $g_{w_{L}, \varphi}: \operatorname{step}\left(\mathfrak{L}\left(w_{L}\right)\right) \rightarrow \mathfrak{F}(s)$ of $\mathfrak{L}_{c}\left(w_{L}\right)$, as in Proposition 8.5, we have

$$
g_{\varphi}(p)=g_{w_{L}, \varphi}(\lambda(p)),
$$

for every step point $p$ of $L$. 
Let $q$ and $r$ be step points such that $q<r$. Suppose that $\lambda(q) \geq \lambda(r)$. Then, for the pair $(\varphi, s)$ considered so far, and in view of $(13.5)$, we may consider in $\mathcal{T}(s)$ an edge $g_{\varphi}(r) \stackrel{t}{\rightarrow} g_{\varphi}(q)$ labeled by some $t \in S^{I}$. On the other hand, according to condition (W.3), there is in $\mathcal{T}(s)$ an edge $g_{\varphi}(q) \stackrel{z}{\rightarrow} g_{\varphi}(r)$ labeled by some $z \in S$. It follows that there is a loop in $\mathcal{T}(s)$ at $g_{\varphi}(q)$ labeled by $z t \in S$. This contradicts (W.4). Hence, we have $\lambda(q)<\lambda(r)$.

It remains to show that $\lambda$ is onto. Let $(u, v)$ be a step point of $\mathfrak{L}\left(w_{L}\right)$. Consider the set

$$
X=\{q \in \operatorname{step}(L): \lambda(q) \leq(u, v)\} .
$$

Notice that $X$ is nonempty: indeed, one clearly has $\min L \in X$.

We claim that $p=\sup X$ is a step point. Suppose not. Let $\varphi: A \rightarrow S$ be the generating mapping of a finite aperiodic unambiguous $A$-generated semigroup. Since $\operatorname{Im} g_{\varphi}$ is finite and $\{q \in \operatorname{step}(L): q>p\}$ is right cofinal at $p$, there is $\left(s_{1}, s_{2}\right) \in \operatorname{Im} g_{\varphi}$ such that

$$
R=\left\{q \in \operatorname{step}(L): q>p \text { and } g_{\varphi}(q)=\left(s_{1}, s_{2}\right)\right\}
$$

is right cofinal at $p$. In particular, $g_{\varphi}^{-1}\left(s_{1}, s_{2}\right)$ is right cofinal at $p$. Taking into account condition (R.4) in Definition 8.2, we know that $g_{\varphi}^{-1}\left(s_{1}, s_{2}\right)$ is also left cofinal at $p$. Therefore, there is a step point $q$ such that $q<p$ and $g_{\varphi}(q)=\left(s_{1}, s_{2}\right)$. Since $p=\sup X$, there is a step point $q^{\prime}$ such that $q<q^{\prime}<p$ and $\lambda\left(q^{\prime}\right) \leq(u, v)$. We have already shown that $\lambda$ is injective and respects the order, so we actually have $\lambda(q)<(u, v)$. Let $r$ be an element of the nonempty set $R$. Since $r>p$, we have $(u, v)<\lambda(r)$. Let $t_{1}$ and $t_{2}$ be (the unique) transitions from $\lambda(q)$ to $(u, v)$ and from $(u, v)$ to $\lambda(r)$, respectively. Then, in $\mathcal{T}(s)$, we have the following edges

$$
g_{w_{L}, \varphi}(\lambda(q)) \stackrel{\varphi_{\mathrm{A}}\left(t_{1}\right)}{\longrightarrow} g_{w_{L}, \varphi}(u, v) \stackrel{\varphi_{\mathrm{A}}\left(t_{2}\right)}{\longrightarrow} g_{w_{L}, \varphi}(\lambda(r)) .
$$

But we have $g_{w_{L}, \varphi}(\lambda(q))=g_{\varphi}(q)=\left(s_{1}, s_{2}\right)=g_{\varphi}(r)=g_{w_{L}, \varphi}(\lambda(r))$. Hence, we can multiply the second edge in (13.6) with the first edge, obtaining a loop at $g_{w_{L}, \varphi}(u, v)=\left(\varphi_{\mathrm{A}}(u), \varphi_{\mathrm{A}}(v)\right)$ labeled by $\varphi_{\mathrm{A}}\left(t_{2} t_{1}\right) \in S$, leading to a contradiction, since $\mathfrak{L}_{c}\left(w_{L}\right)$ satisfies (W.4). This establishes the claim that $p$ is a step point, thus $p \in X$.

Suppose that $\lambda(p)<(u, v)$. Let $p^{\prime}$ be the step point such that $p \prec p^{\prime}$. Then, applying (13.4), we get $\lambda(p) \prec \lambda\left(p^{\prime}\right)$. Since $\lambda(p)<(u, v)$, we obtain $\lambda\left(p^{\prime}\right) \leq(u, v)$, and so $p^{\prime} \in X$. But then $p^{\prime} \leq \sup X=p$, a contradiction with $p<p^{\prime}$. As $p \in X$, to avoid the contradiction, we must have $\lambda(p)=(u, v)$. This concludes the proof that $\lambda$ is onto.

It would also be interesting to characterize the worthy clustered linear orders that arise as the images $\mathfrak{L}_{c}(w)$ of $\omega$-words $w$. We leave this as an open problem. 


\section{On the cardinality of the set of stationary points}

Let $\mathrm{V}$ be an equidivisible pseudovariety of semigroups not contained in CS. Then $\mathrm{V}$ is finitely cancelable (cf. Proposition 6.4), and so, by Theorem 7.4, for a finite alphabet $A$ and for $w \in \bar{\Omega}_{A} \bigvee$, the set $\operatorname{step}(\mathfrak{L}(w))$ of step points is the set of isolated points of $\mathfrak{L}(w)$, with respect to the order topology. Therefore, $\operatorname{step}(\mathfrak{L}(w))$ is at most countable by Corollary 4.4. The aim of this section is to show that when $A$ has at least two elements, there are elements $w$ in $\bar{\Omega}_{A} \vee$ for which the set $\operatorname{stat}(\mathfrak{L}(w))$ of stationary points has cardinal $2^{\aleph_{0}}$. This will be done using some tools originated from symbolic dynamics, following an approach that has been successfully used in recent years to elucidate structural aspects of relatively free profinite semigroups $[\mathbf{2}, \mathbf{9}, \mathbf{4}, \mathbf{1 7}, \mathbf{5}]$.

14.1. Subshifts. Consider a finite alphabet $A$, and endow $A^{\mathbb{Z}}$ with the product topology, where $A$ is endowed with the discrete topology. The shift map of $A^{\mathbb{Z}}$ is the homeomorphism $\sigma: A^{\mathbb{Z}} \rightarrow A^{\mathbb{Z}}$, defined by $\sigma\left(\left(x_{i}\right)_{i \in \mathbb{Z}}\right)=\left(x_{i+1}\right)_{i \in \mathbb{Z}}$. A symbolic dynamical system of $A^{\mathbb{Z}}$, also called subshift of $A^{\mathbb{Z}}$, is a nonempty closed subset $X$ of $A^{\mathbb{Z}}$ such that $\sigma(X)=X$. The books $[\mathbf{3 0}, \mathbf{2 7}]$ are good references on symbolic dynamical systems.

We say that a subset $L$ of a semigroup $S$ is

- factorial if it is closed under taking factors;

- prolongable if, for every $s \in L$, there are $t, u \in S$ such that $t s, s u \in L$;

- irreducible if, for all $s, t \in L$, there is $u \in S$ such that $s u t \in L$.

If $X$ is a subshift of $A^{\mathbb{Z}}$, then $L(X)$ denotes the language of the words of $A^{+}$of the form $x_{k} x_{k+1} \ldots x_{k+n}$, where $k \in \mathbb{Z}, n \geq 0$, and $\left(x_{i}\right)_{i \in \mathbb{Z}} \in X$. The set $L(X)$ is a factorial and prolongable language of $A^{+}$, and in fact all nonempty factorial and prolongable languages of $A^{+}$are of this form; moreover, $y \subseteq X$ if and only if $L(y) \subseteq L(X)$, whenever $X$ and $y$ are subshifts of $A^{\mathbb{Z}}$ [30, Proposition 1.3.4]. Finally, $X$ is said to be irreducible if $L(X)$ is an irreducible subset of $A^{+}$.

If $X$ is a subshift of $A^{\mathbb{Z}}$ then the sequence $\left(\frac{1}{n} \log _{2}\left|L(X) \cap A^{n}\right|\right)_{n}$ converges to its infimum, which is called the entropy of $X$ and denoted $h(X)[\mathbf{3 0}]$. Note that $X \subseteq y$ implies $h(X) \leq h(y)$, whenever $X$ and $y$ are subshifts.

Remark 14.1. If $X$ is a subshift of $A^{\mathbb{Z}}$ then $h(X) \leq \log _{2}|A|=h\left(A^{\mathbb{Z}}\right)$. Moreover, from the fact that $\left(\frac{1}{n} \log _{2}\left|L(X) \cap A^{n}\right|\right)_{n}$ converges to its infimum one easily deduces that the subshift $X$ of $A^{\mathbb{Z}}$ satisfies $h(X)=$ $\log _{2}|A|$ if and only if $\mathcal{X}=A^{\mathbb{Z}}$ (this a special case of [30, Corollary 4.4.9]). 
14.2. A special $\mathcal{J}$-class. Consider a subshift $\mathcal{X}$ of $A^{\mathbb{Z}}$, and suppose that $\mathrm{V}$ is a pseudovariety containing LSI. Let $M_{\mathrm{V}}(X)$ be the set of pseudowords $w \in \bar{\Omega}_{A} \vee$ such that all finite factors of $w$ belong to $L(X)$. The set $M_{\mathrm{V}}(\mathcal{X})$ is a factorial subset of $\bar{\Omega}_{A} \mathrm{~V}$. Because, as it is well known, the languages of the form $A^{*} u A^{*}$, with $u \in A^{+}$, are LSI-recognizable, the hypothesis that $\mathrm{V}$ contains $\mathrm{LSI}$ ensures that $M_{\mathrm{V}}(X)$ is a closed subset of $\bar{\Omega}_{A} \vee$ (cf. [16]).

Lemma 14.2. Let $X$ be an irreducible subshift of $A^{\mathbb{Z}}$. Consider a pseudovariety $\mathrm{V}$ containing LSI. For every $u, v \in M_{\mathrm{V}}(X)$ there is $w \in \bar{\Omega}_{A} \mathrm{~V}$, depending only on the finite suffixes of $u$ and on the finite prefixes of $v$, such that $u w v \in M_{\mathrm{V}}(X)$.

Proof: If $\mathrm{V}$ is a pseudovariety containing $\mathrm{D}$ and its dual, then every infinite element of $\bar{\Omega}_{A} \mathrm{~V}$ has a unique prefix (suffix) in $A^{+}$with length $n$, for every $n \geq 1$. Let $s_{n}$ be the suffix of length $n$ of $u$ and let $p_{n}$ be the prefix of length $n$ of $v$. Since $X$ is irreducible, for each $n \in \mathbb{N}$, there is $w_{n} \in L(X)$ such that $s_{n} w_{n} p_{n} \in L(X)$. Let $w$ be an accumulation point of $\left(w_{n}\right)_{n}$. Then $w$ has the desired property.

Proposition 14.3 ([17, Proposition 3.6]). Let $S$ be a compact semigroup and let $X \subseteq S$. Then $X$ is a closed, factorial, irreducible subset of $S$ if and only if $X$ consists of all factors of some regular element of $S$.

By Lemma 14.2 and Proposition 14.3, if $X$ is an irreducible subshift, then there is a unique regular $\mathcal{J}$-class $J_{V}(X)$ such that the elements of $M_{\mathrm{V}}(X)$ are the factors of elements of $J_{\mathrm{V}}(X)$.

Remark 14.4. It also follows from Proposition 14.3 that $J_{\mathrm{V}}(X) \leq_{\mathcal{J}} J_{\mathrm{V}}(y)$ if and only if $M_{\mathrm{V}}(\mathrm{y}) \subseteq M_{\mathrm{V}}(\mathcal{X})$. Since we clearly have $M_{\mathrm{V}}(\mathrm{y}) \subseteq M_{\mathrm{V}}(\mathcal{X})$ if and only if $L(y) \subseteq L(X)$, we conclude that

$$
y \subseteq X \Longleftrightarrow J_{\mathrm{V}}(X) \leq_{\mathcal{J}} J_{\mathrm{V}}(y) .
$$

Remark 14.5. If $X=A^{\mathbb{Z}}$ then $M_{\mathrm{V}}(X)=\bar{\Omega}_{A} \mathrm{~V}$, and so $J_{\mathrm{V}}(X)$ is the minimum ideal of $\bar{\Omega}_{A} \mathrm{~V}$.

14.3. Uncountable $<_{\mathcal{R}}$-chains and uncountable sets of stationary points. We use the standard notation $\lceil\alpha\rceil$ for the least integer greater than or equal to the real number $\alpha$.

Theorem 14.6. There is a family $\left(\mathcal{S}_{\beta}\right)_{\beta \in] 1,+\infty[}$ of symbolic dynamical systems, parameterized by the set of real numbers greater than one, such that:

(a) $\mathcal{S}_{\beta}$ is an irreducible subshift of $\{0, \ldots,\lceil\beta\rceil-1\}^{\mathbb{Z}}$;

(b) $h\left(\mathcal{S}_{\beta}\right)=\log _{2} \beta$;

(c) for every $\alpha, \beta \in] 1,+\infty$ [, we have $\alpha<\beta$ if and only if $\mathcal{S}_{\alpha} \subsetneq \mathcal{S}_{\beta}$. 
A concrete family of symbolic dynamical systems satisfying the conditions of Theorem 14.6 is the family of $\beta$-shifts. A comprehensive exposition about this family can be found in [31, Chapter 7] and [20]. That these subshifts are irreducible follows from them being coded [11] - a subshift $X$ of $A^{\mathbb{Z}}$ is coded if there is a prefix code $Y$ contained in $A^{+}$ such that $L(\mathcal{X})$ is the set of factors of elements of $Y^{+}$. The entropy of $\beta$-shifts was computed in $[\mathbf{3 8}, \mathbf{3 4}]$. The fact that this class fits into property (c) of Theorem 14.6 appears at the beginning of [25, Section 4] (actually, only the implication $\alpha \leq \beta \Rightarrow \mathcal{S}_{\alpha} \subseteq \mathcal{S}_{\beta}$ is explicit there, but from property (b) one gets $\mathcal{S}_{\alpha} \subsetneq \mathcal{S}_{\beta} \Rightarrow \alpha<\beta$ ).

As usual, the notation $<\mathcal{J}$ stands for the irreflexive relation originated by $\leq_{\mathcal{J}}$, and similarly for $<_{\mathcal{R}}$ and $<_{\mathcal{L}}$. From Theorem 14.6 and equivalence (14.1) in Remark 14.4 one immediately deduces the existence of a $<_{\mathfrak{g}}$-chain in $\bar{\Omega}_{A} \mathrm{~V}$ formed by $2^{\aleph_{0}}$ regular elements, whenever $\mathrm{V}$ contains LSI and $A$ has at least two letters. The next theorem gives a refinement of this, as it shows in particular the existence in $\bar{\Omega}_{A} \mathrm{~V}$ of a $<_{\mathcal{R}}$-chain formed by $2^{\aleph_{0}}$ regular elements. We remark that in $[\mathbf{1 8}]$ an example is given of a $<_{\mathcal{R}}$-chain of $2^{\aleph_{0}}$ non-regular elements in $\bar{\Omega}_{A} \mathrm{LSI}$, when $|A|>1$.

Theorem 14.7. Let $\mathrm{V}$ be a finitely cancelable pseudovariety of semigroups containing $\mathrm{LSI}$ and let $\left(\mathcal{S}_{\beta}\right)_{\beta \in] 1,+\infty[}$ be a family of subshifts as in Theorem 14.6. Fix an integer $n>1$ and let $A$ be the alphabet $\{0, \ldots, n-$ $1\}$. There is a family $\left(w^{(\beta)}\right)_{\beta \in] 1, n]}$ of pseudowords of $\bar{\Omega}_{A} \vee$ satisfying the following conditions:

(a) $w^{(\beta)} \in J_{\vee}\left(\mathcal{S}_{\beta}\right) \subseteq \bar{\Omega}_{A} \vee$, for every $\left.\left.\beta \in\right] 1, n\right]$;

(b) $\alpha<\beta \Leftrightarrow w^{(\beta)}<_{\mathcal{R}} w^{(\alpha)}$, for every $\left.\left.\alpha, \beta \in\right] 1, n\right]$;

(c) $w^{(n)}$ is an element of the minimum ideal of $\bar{\Omega}_{A} \mathrm{~V}$;

(d) there is a subnet of $\left(w^{(\beta)}\right)_{\beta \in] 1, n[}$ converging to $w^{(n)}$, where $] 1, n[$ is endowed with the usual order;

(e) for each $\beta \in] 1, n]$, there are $v^{(\beta)}, f^{(\beta)}$ such that $\left(w^{(\beta)}, v^{(\beta)}\right)$ is a stationary point of $\mathfrak{F}\left(w^{(n)}\right)$, and, for $q_{\beta}=\left(w^{(\beta)}, v^{(\beta)}\right) / \sim$, the pseudoword $f^{(\beta)}$ is an idempotent in $J_{q_{\beta}}$ stabilizing $\left(w^{(\beta)}, v^{(\beta)}\right)$ and satisfying $f^{(\beta)} \mathcal{L} w^{(\beta)}$;

(f) we have $\alpha<\beta \Rightarrow q_{\alpha}<q_{\beta}$, and if moreover $\bar{\Omega}_{A} \mathrm{~V}$ is equidivisible, then the equivalence $\alpha<\beta \Leftrightarrow q_{\alpha}<q_{\beta}$ holds, for every $\left.\left.\alpha, \beta \in\right] 1, n\right]$.

The proof of Theorem 14.7 will be done in several steps. But first we highlight the following corollary, which is our main motivation for the theorem. 
Corollary 14.8. Let $\mathrm{V}$ be a finitely cancelable pseudovariety of semigroups containing $\mathrm{LSI}$ and let $A$ be a finite alphabet with at least two elements. Then there are pseudowords $w$ in the minimum ideal of $\bar{\Omega}_{A} \mathrm{~V}$ such that $\operatorname{stat}(\mathfrak{L}(w))$ has $2^{\aleph_{0}}$ elements.

Note also that Theorem 14.7 gives an example of a pseudoword in the minimum ideal of $\bar{\Omega}_{A} \mathrm{~V}$ whose set of stationary points contains a subset with the same order type as the set of real numbers. In contrast, the following example exhibits a pseudoword also in the minimum ideal of $\bar{\Omega}_{A} \mathrm{~V}$ with only one stationary point. As was pointed out by the anonymous referee, all saturated labeled linear orders considered in [21] for elements of the minimum ideal of $\bar{\Omega}_{A} \mathrm{~A}$ are uncountable.

Example 14.9. Let $u_{1}, u_{2}, u_{3}, \ldots$ be an enumeration of the elements of $A^{+}$, and let $\mathrm{V}$ be a pseudovariety containing $\mathrm{LSI}$ such that $\bar{\Omega}_{A} \mathrm{~V}$ is equidivisible. For each $k \geq 1$, consider in $\bar{\Omega}_{A} \mathrm{~V}$ an accumulation point $v_{k}$ of the sequence $\left(u_{k} u_{k+1} \cdots u_{n-1} u_{n}\right)_{n \geq k}$ and an accumulation point $w_{k}$ of the sequence $\left(u_{n} u_{n-1} \cdots u_{k+1} u_{k}\right)_{n \geq k}$. As every element of $A^{+}$is a factor of $v_{k}$ and $w_{k}$, we know that $v_{k}$ and $w_{k}$ belong to the minimum ideal $K_{A}$ of $\bar{\Omega}_{A} \mathrm{~V}$. Therefore, if $p$ and $q$ are respectively the first and last stationary point of $\mathfrak{L}\left(v_{1} w_{1}\right)$, then $J_{p}=J_{q}=K_{A}$ by Theorem 10.1 , and so $p=q$ by Lemma 7.9 .

14.4. About the proof of Theorem 14.7. Let $S$ be a compact semigroup and $I$ an ordered set. Suppose that $F=\left(F_{i}\right)_{i \in I}$ is a nonempty family of compact subsets of $S$. Denote by $R_{F}$ the set of partial functions $f$ from $I$ to $\bigcup F$ such that $f(i) \in F_{i}$ for all $i \in \operatorname{Dom} f$, and such that $i \leq j \Rightarrow f(i) \leq_{\mathcal{R}} f(j)$ whenever $i, j \in \operatorname{Dom} f$. We endow $R_{F}$ with the partial order $\leq$ defined by

$$
f \leq g \Longleftrightarrow(f=g \vee \operatorname{Dom} f \subsetneq \operatorname{Dom} g) .
$$

Lemma 14.10. The ordered set $R_{F}$ has a maximal element.

Proof: Let $C$ be a chain of elements of $R_{F}$. We want to show that $C$ has an upper bound in $R_{F}$. For each $f \in R_{F}$, let $f^{\prime}$ be an element of $\prod_{i \in I} F_{i}$ whose restriction to $\operatorname{Dom} f$ equals $f$. Since $\prod_{i \in I} F_{i}$ is compact, the net $\left(f^{\prime}\right)_{f \in C}$ has a subnet converging to some $\varphi \in \prod_{i \in I} F_{i}$. For achieving our goal, we may as well assume that $\left(f^{\prime}\right)_{f \in C}$ converges. Let us fix an element $f_{0}$ of $C$, and take $i, j \in \operatorname{Dom} f_{0}$ such that $i \leq j$. For all $f \in C$ such that $f_{0} \leq f$, one has $f(i) \leq_{\mathcal{R}} f(j)$. As the net $\left(f^{\prime}\right)_{f \in C \wedge f_{0} \leq f}$ converges to $\varphi$ and $\leq_{\mathcal{R}}$ is a closed relation, we deduce that $\varphi(i) \leq_{\mathcal{R}} \varphi(j)$. Moreover, since $F_{i}$ is closed, we also have $\varphi(k) \in F_{k}$ for all $k \in \operatorname{Dom} f_{0}$. As $f_{0}$ was chosen arbitrarily from $C$, we conclude that the restriction 
of $\varphi$ to $\bigcup_{f \in C}$ Dom $f$ belongs to $R_{F}$ and is an upper bound for $C$. Hence, by Zorn's Lemma, $R_{F}$ has a maximal element.

Proposition 14.11. For the relation $\supseteq$, let $\mathcal{C}$ be a nonempty chain of irreducible subshifts of $A^{\mathbb{Z}}$. Consider a pseudovariety of semigroups $\mathrm{V}$ that contains LSI. Let $\mathscr{J}$ be the family of $\mathcal{J}$-classes $\left(J_{\mathrm{V}}(X)\right)_{x \in \mathcal{C}}$. Then there is an element of $R_{\mathscr{J}}$ with domain $\mathcal{C}$.

Proof: By Lemma 14.10, we know there is in $R_{\mathscr{J}}$ a maximal element $f$. We claim that Dom $f=\mathcal{C}$. Suppose this is false. Let $z \in \mathcal{C} \backslash \operatorname{Dom} f$. Supposing that $I=\{X \in \operatorname{Dom} f: X \subseteq Z\}$ is nonempty, let $u$ be an accumulation point of the net $(f(\mathcal{X}))_{X \in(I, \subset)}$; in case $I=\emptyset$, we let $u$ be any element of $J_{\vee}(Z)$. Since $X \subseteq Z$ implies $M_{\vee}(X) \subseteq M_{\vee}(Z)$, we have $f(X) \in M_{\vee}(\mathcal{Z})$ for all $X \in I$. And since $M_{\vee}(\mathcal{Z})$ is closed, we conclude that $u \in M_{\vee}(Z)$. Moreover, fixed $X \in I$, then, as $f \in R_{\mathscr{J}}$, we have $f(y) \leq_{\mathcal{R}} f(X)$ for all $y \in I$ such that $X \subseteq y$, whence

$$
X \in I \Longrightarrow u \leq_{\mathcal{R}} f(X) \text {. }
$$

Let $v \in J_{\mathrm{V}}(Z)$. By the irreducibility of $M_{\mathrm{V}}(Z)$, there is $w \in \bar{\Omega}_{A} \vee$ such that $u w v \in M_{\vee}(Z)$. Since $u w v$ is a factor of $v$ and $v$ is a $\mathcal{J}$-minimum element of $M_{\mathrm{V}}(Z)$, we have $u w v \in J_{\vee}(Z)$. As $f \in R_{\mathscr{J}}$, every two elements in the image of $f$ are $\mathcal{R}$-comparable, and so the elements in the image of $f$ have all the same set $P$ of finite prefixes. By Lemma 14.2, for each $X \in \operatorname{Dom} f$ such that $\mathcal{Z} \subseteq \mathcal{X}$, there is a pseudoword $w^{\prime}$, depending only on $u w v$ and $P$, such that $u w v w^{\prime} f(X) \in M_{\vee}(X)$. More precisely, we have $u w v w^{\prime} f(X) \in J_{\vee}(X)$, as $f(X) \in J_{\vee}(X)$. The partial function

$$
f^{\prime}: X \in \operatorname{Dom} f \cup\{z\} \mapsto \begin{cases}f(X) & \text { if } x \subsetneq z, \\ u w v & \text { if } X=z, \\ u w v w^{\prime} f(X) & \text { if } z \subsetneq \mathcal{X}\end{cases}
$$

belongs to $R_{\mathscr{J}}$ (cf. implication (14.2)) and $\operatorname{Dom} f \subsetneq \operatorname{Dom} f^{\prime}$. This contradicts the fact that $f$ is a maximal element of $R_{\mathscr{J}}$. The absurdity comes from the hypothesis $\mathrm{e} \backslash \operatorname{Dom} f \neq \emptyset$.

We recall the concept of entropy of a pseudoword, first introduced in [9], and applied there in the study of relatively free profinite semigroups. Some further applications were given in [17]. Let $\mathrm{V}$ be a pseudovariety containing LSI and $A$ an alphabet with at least two letters. For $w \in \bar{\Omega}_{A} \mathrm{~V}$, let $q_{w}(n)$ denote the number of factors of length $n$ of $w$. If $w$ is an infinite pseudoword then the sequence $\frac{1}{n} \log _{2} q_{w}(n)$ converges to its 
infimum, which is denoted by $h(w)$ and called the entropy of $w .^{1}$ This definition extends to finite words, by letting $h(w)=0$ when $w$ is finite. If $X$ is a subshift, then $h(X)=h(w)$ for every pseudoword $w$ whose set of finite factors is equal to $L(\mathcal{X})$. For instance, if $\mathcal{X}$ is irreducible then $h(X)=h(w)$ when $w \in J_{\mathrm{V}}(X)$.

Note that $h(w) \in\left[0, \log _{2}|A|\right]$, for all $w \in \bar{\Omega}_{A} \vee$. Moreover, we have the following fact from $[\mathbf{9}]$.

Proposition 14.12. Let $w \in \bar{\Omega}_{A} \vee$. Then $h(w)=\log _{2}|A|$ if and only if $w$ belongs to the minimum ideal of $\bar{\Omega}_{A} \mathrm{~V}$.

In particular, the entropy of pseudowords of $\bar{\Omega}_{A} \mathrm{~V}$ is not continuous, since every finite word has entropy zero and the set of finite words is dense. However, it is upper semi-continuous, as proved next.

Lemma 14.13. Let $\mathrm{V}$ be a pseudovariety containing LSI. If $\left(w_{n}\right)_{n}$ is a sequence of elements of $\bar{\Omega}_{A} \mathrm{~V}$ converging to $w$ then $\lim \sup h\left(w_{n}\right) \leq h(w)$.

Proof: Since limsup $h\left(w_{n}\right)$ is the greatest accumulation point of the sequence $\left(h\left(w_{n}\right)\right)_{n}$, the proof is reduced to the case where $\left(h\left(w_{n}\right)\right)_{n}$ converges.

Since $\lim w_{n}=w$ and $\mathrm{V}$ contains LSI, for each $k$ there is $p_{k}$ such that for all $n \geq p_{k}$ the pseudowords $w_{n}$ and $w$ have the same factors of length $k$. Let $\left(n_{k}\right)_{k}$ be the sequence recursively defined by $n_{1}=p_{1}$ and $n_{k+1}=\max \left\{n_{k}, p_{k+1}\right\}$. Given $\varepsilon>0$, consider the set $K=\{k$ : $\left.h\left(w_{n_{k}}\right) \geq h(w)+\varepsilon\right\}$. For every $k \in K$, one has

$$
\frac{1}{k} \log _{2} q_{w}(k)=\frac{1}{k} \log _{2} q_{w_{n_{k}}}(k) \geq h\left(w_{n_{k}}\right) \geq h(w)+\varepsilon .
$$

As $\lim \frac{1}{k} \log _{2} q_{w}(k)=h(w)$, if $K$ is infinite then (14.3) leads to the contradiction $h(w) \geq h(w)+\varepsilon$. Hence $K$ is finite, and so $\lim h\left(w_{n_{k}}\right) \leq$ $h(w)+\varepsilon$. Since $\varepsilon$ is arbitrary and $\left(h\left(w_{n}\right)\right)_{n}$ converges, we conclude that $\lim h\left(w_{n}\right) \leq h(w)$.

We now have all the tools to achieve the proof of Theorem 14.7.

Proof of Theorem 14.7: Let $\mathcal{C}$ be the chain $\left(\mathcal{S}_{\beta}\right)_{] 1, n}[$, ordered by $\supseteq$. Applying Proposition 14.11 to the family of $\mathcal{J}$-classes $\left(J_{\vee}(X)\right) x_{\in} \mathcal{e}$, we conclude that there is a function $f: \mathcal{C} \rightarrow \bar{\Omega}_{A} \vee$ such that $f\left(\mathcal{S}_{\beta}\right) \in J_{\vee}\left(\mathcal{S}_{\beta}\right)$ and

$$
\left.\mathcal{S}_{\beta} \supseteq \mathcal{S}_{\alpha} \Longrightarrow f\left(\mathcal{S}_{\beta}\right) \leq \mathcal{R} f\left(\mathcal{S}_{\alpha}\right), \quad \forall \alpha, \beta \in\right] 1, n[.
$$

\footnotetext{
${ }^{1}$ This is the definition used in [17]. In [9] the entropy of $w$ is defined as $\frac{1}{n} \log _{|A|} q_{w}(n)$, which equals $h(w) \log _{|A|} 2$ for $h(w)$ as defined here.
} 
On the other hand, if $f\left(\mathcal{S}_{\beta}\right) \leq_{\mathcal{R}} f\left(\mathcal{S}_{\alpha}\right)$, then $\mathcal{S}_{\beta} \supseteq \mathcal{S}_{\alpha}$ by equivalence (14.1) in Remark 14.4. Therefore, we actually have

$$
\left.\mathcal{S}_{\beta} \supsetneq \mathcal{S}_{\alpha} \Longleftrightarrow f\left(\mathcal{S}_{\beta}\right)<_{\mathcal{R}} f\left(\mathcal{S}_{\alpha}\right), \quad \forall \alpha, \beta \in\right] 1, n[.
$$

For each $\beta \in] 1, n\left[\right.$, let $w^{(\beta)}=f\left(\mathcal{S}_{\beta}\right)$. By the given characterization of $\left(\mathcal{S}_{\beta}\right)_{\beta \in] 1,+\infty}$ [ (cf. Theorem 14.6), we know that $\mathcal{S}_{\beta} \supsetneq \mathcal{S}_{\alpha}$ if and only if $\alpha<\beta$, whence (14.4) translates to

$$
\left.\alpha<\beta \Longleftrightarrow w^{(\beta)}<_{\mathcal{R}} w^{(\alpha)}, \quad \forall \alpha, \beta \in\right] 1, n[.
$$

Let $\left(\alpha_{k}\right)_{k}$ be an increasing sequence of elements of the open interval $] 1, n[$ such that $\lim \alpha_{k}=n$. Thus, we have $\lim h\left(w^{\left(\alpha_{k}\right)}\right)=\lim \log _{2} \alpha_{k}=$ $\log _{2} n$. Hence, if $w^{(n)}$ is an accumulation point of the sequence $\left(w^{\left(\alpha_{k}\right)}\right)_{k}$, then $h\left(w^{(n)}\right)=\log _{2} n$ by Lemma 14.13 and Remark 14.1. By Proposition 14.12, the pseudoword $w^{(n)}$ then belongs to the minimum ideal of $\bar{\Omega}_{A} \mathrm{~V}$. Since $\leq_{\mathcal{R}}$ is a closed relation, we have $w^{(n)} \leq_{\mathcal{R}} w^{(\beta)}$ for all $\beta \in] 1, n[$. Then, taking into account (14.5), we conclude that the net $\left(w^{(\beta)}\right)_{\beta \in] 1, n]}$ satisfies conditions (a)-(d) in Theorem 14.7.

As $w^{(n)} \leq_{\mathcal{R}} w^{(\beta)}$, there is $u^{(\beta)}$ with $w^{(n)}=w^{(\beta)} u^{(\beta)}$. Since $w^{(\beta)}$ is regular, there is an idempotent $f^{(\beta)}$ in the $\mathcal{L}$-class of $w^{(\beta)}$. Take $v^{(\beta)}=f^{(\beta)} u^{(\beta)}$. Then $\left(w^{(\beta)}, v^{(\beta)}\right)$ is an element of $\mathfrak{F}\left(w^{(n)}\right)$ stabilized by $f^{(\beta)}$. By Proposition 7.5, this implies that $q_{\beta}=\left(w^{(\beta)}, v^{(\beta)}\right) / \sim$ is a stationary point. The elements of $J_{q_{\beta}}$ are factors of $w^{(\beta)}$, and so by the minimality of $J_{q_{\beta}}$ we have $f^{(\beta)} \in J_{q_{\beta}}$. Hence, condition (e) in Theorem 14.7 holds.

Let $\alpha, \beta \in] 1, n]$. Since $q_{\alpha} \leq q_{\beta} \Rightarrow w^{(\beta)} \leq_{\mathcal{R}} w^{(\alpha)}$, we deduce from (14.5) that $q_{\alpha} \leq q_{\beta} \Rightarrow \alpha \leq \beta$. Thus, if $\alpha<\beta$ then we cannot have $q_{\beta} \leq q_{\alpha}$, and so assuming $\bar{\Omega}_{A} \mathrm{~V}$ is equidivisible, we get $q_{\alpha}<q_{\beta}$, thereby establishing condition (f) in Theorem 14.7.

\section{Acknowledgments}

The authors wish to thank the anonymous referee for numerous comments and suggestions that contributed to the improvement of the presentation of our results.

The work of the first, third, and fourth authors was partly supported by the PessoA French-Portuguese project "Separation in automata theory: algebraic, logical, and combinatorial aspects". The work of the first three authors was also partially supported respectively by CMUP (UID/MAT/ 00144/2019), CMUC (UID/MAT/00324/2019), and CMAT (UID/MAT/ 00013/2013), which are funded by FCT (Portugal) with national (MCTES) and European structural funds (FEDER), 
under the partnership agreement PT2020. The work of the fourth author was partly supported by ANR 2010 BLAN 020201 FREC and by the DeLTA project ANR-16-CE40-0007.

\section{References}

[1] J. Almeida, "Finite Semigroups and Universal Algebra", Translated from the 1992 Portuguese original and revised by the author, Series in Algebra 3, World Scientific Publishing Co., Inc., River Edge, NJ, 1994. DOI : 10.1142/2481.

[2] J. Almeida, Profinite groups associated with weakly primitive substitutions, (Russian), Fundam. Prikl. Mat. 11(3) (2005), 13-48; translation in: J. Math. Sci. (N.Y.) 144(2) (2007), 3881-3903. DOI : 10.1007/s10958-007-0242-y.

[3] J. AlmeidA, Profinite semigroups and applications. Notes taken by Alfredo Costa, in: "Structural Theory of Automata, Semigroups, and Universal Algebra", NATO Sci. Ser. II Math. Phys. Chem. 207, Springer, Dordrecht, 2005, pp. 1-45. DOI : $10.1007 / 1-4020-3817-8_{-} 1$.

[4] J. Almeida And A. Costa, Infinite-vertex free profinite semigroupoids and symbolic dynamics, J. Pure Appl. Algebra 213(5) (2009), 605-631. DOI: $10.1016 / j \cdot j$ paa. 2008.08.009.

[5] J. Almeida And A. Costa, Presentations of Schützenberger groups of minimal subshifts, Israel J. Math. 196(1) (2013), 1-31. DOI: 10.1007/s11856-0120139-4.

[6] J. Almeida And A. Costa, Equidivisible pseudovarieties of semigroups, Publ. Math. Debrecen 90(3-4) (2017), 435-453. DOI : 10.5486/PMD. 2017.7634.

[7] J. Almeida and O. Klíma, Representations of relatively free profinite semigroups, irreducibility, and order primitivity, Preprint (2015). arXiv:1509.01389.

[8] J. Almeida And M. V. Volkov, Profinite methods in finite semigroup theory, in: Proceedings of International Conference "Logic and Applications" honoring Yu. L. Ershov on his 60-th birthday and of International Conference on Mathematical Logic, honoring A. I. Mal'tsev on his 90-th birthday, and 275-th anniversary of the Russian Academy of Sciences (Novosibirsk, Russia) (S. S. Goncharov, ed.), 2002, pp. 3-28.

[9] J. Almeida And M. V. Volkov, Subword complexity of profinite words and subgroups of free profinite semigroups, Internat. J. Algebra Comput. 16(2) (2006), 221-258. DOI : 10.1142/S0218196706002883.

[10] J. Almeida And P. Weil, Free profinite $\mathscr{R}$-trivial monoids, Internat. J. Algebra Comput. 7(5) (1997), 625-671. DOI : 10.1142/S0218196797000289.

[11] A. Bertrand-Mathis, Développement en base $\theta$; répartition modulo un de la suite $\left(x \theta^{n}\right)_{n \geq 0}$; langages codés et $\theta$-shift, Bull. Soc. Math. France 114(3) (1986), 271-323. DOI : 10.24033/bsmf . 2058.

[12] J.-C. Birget, Iteration of expansions-unambiguous semigroups, J. Pure Appl. Algebra 34(1) (1984), 1-55. DOI : 10.1016/0022-4049(84)90055-0.

[13] V. Bruyère and O. Carton, Automata on linear orderings, J. Comput. System Sci. 73(1) (2007), 1-24. DOI : 10.1016/j.jcss.2006.10.009.

[14] J. H. Carruth, J. A. Hildebrant, and R. J. Koch, "The Theory of Topological Semigroups", Monographs and Textbooks in Pure and Applied Mathematics 75, Marcel Dekker, Inc., New York, 1983. 
[15] L. Chaubard, J.-É. Pin, and H. Straubing, Actions, wreath products of $\mathscr{C}$ varieties and concatenation product, Theoret. Comput. Sci. 356(1-2) (2006), 73-89. DOI : $10.1016 / \mathrm{j}$.tcs. 2006.01.039.

[16] A. Costa, Semigrupos profinitos e dinâmica simbólica, Ph.D. thesis, Universidade do Porto (2007).

[17] A. Costa And B. Steinberg, Profinite groups associated to sofic shifts are free, Proc. Lond. Math. Soc. (3) 102(2) (2011), 341-369. DOI : 10.1112/plms/pdq024.

[18] J. C. Costa, Free profinite locally idempotent and locally commutative semigroups, J. Pure Appl. Algebra 163(1) (2001), 19-47. DOI: 10.1016/S0022-4049 (00) 00176-6.

[19] S. EilenberG, "Automata, Languages, and Machines", Vol. B, With two chapters ("Depth decomposition theorem" and "Complexity of semigroups and morphisms") by B. Tilson, Pure and Applied Mathematics 59, Academic Press [Harcourt Brace Jovanovich, Publishers], New York-London, 1976.

[20] C. Frougny, Number representation and finite automata, in: "Topics in Symbolic Dynamics and Applications" (Temuco, 1997), London Math. Soc. Lecture Note Ser. 279, Cambridge Univ. Press, Cambridge, 2000, pp. 207-228. DOI: 10.1017/CB09780511758959.008.

[21] S. J. V. Gool And B. Steinberg, Pro-aperiodic monoids via saturated models, in: "34th Symposium on Theoretical Aspects of Computer Science", Art. No. 39, LIPIcs. Leibniz Int. Proc. Inform. 66, Schloss Dagstuhl. Leibniz-Zent. Inform., Wadern, 2017, 14 pp.

[22] P.-A. Grillet, "Semigroups. An Introduction to the Structure Theory", Monographs and Textbooks in Pure and Applied Mathematics 193, Marcel Dekker, Inc., New York, 1995.

[23] K. Henckell, J. Rhodes, and B. Steinberg, A profinite approach to stable pairs, Internat. J. Algebra Comput. 20(2) (2010), 269-285. DOI: $10.1142 / \mathrm{S} 0218196710005650$.

[24] M. Huschenbett And M. Kufleitner, Ehrenfeucht-Fraïssé games on omegaterms, in: "31st International Symposium on Theoretical Aspects of Computer Science”, LIPIcs. Leibniz Int. Proc. Inform. 25, Schloss Dagstuhl. Leibniz-Zent. Inform., Wadern, 2014, pp. 374-385. DOI : 10.4230/LIPIcs.STACS. 2014.374.

[25] S. Ito And Y. TAKahashi, Markov subshifts and realization of $\beta$-expansions, $J$. Math. Soc. Japan 26(1) (1974), 33-55. DOI : 10.2969/jmsj/02610033.

[26] P. T. Johnstone, "Stone Spaces", Reprint of the 1982 edition, Cambridge Studies in Advanced Mathematics 3, Cambridge University Press, Cambridge, 1986.

[27] B. P. Kitchens, "Symbolic Dynamics. One-sided, Two-sided and Countable State Markov Shifts", Universitext, Springer-Verlag, Berlin, 1998. DOI: 10.1007/978-3-642-58822-8.

[28] M. Kufleitner and J. P. Wächter, The word problem for omega-terms over the Trotter-Weil hierarchy (extended abstract), in: "Computer Science-Theory and Applications", Lecture Notes in Comput. Sci. 9691, Springer, Cham, 2016, pp. 237-250. DOI : 10.1007/978-3-319-34171-2_17.

[29] G. Lallement, "Semigroups and Combinatorial Applications", Pure and Applied Mathematics, A Wiley-Interscience Publication, John Wiley \& Sons, New York-Chichester-Brisbane, 1979.

[30] D. Lind And B. Marcus, "An Introduction to Symbolic Dynamics and Coding", Cambridge University Press, Cambridge, 1995. DOI : 10.1017/CB09780511626302. 
[31] M. Lothaire, "Algebraic Combinatorics on Words", With a preface by Berstel and Perrin, Encyclopedia of Mathematics and its Applications 90, Cambridge University Press, Cambridge, 2002. DOI : 10.1017/CB09781107326019.

[32] J. D. McKnight, Jr. And A. J. Storey, Equidivisible semigroups, J. Algebra 12(1) (1969), 24-48. DOI : 10.1016/0021-8693(69) 90015-5.

[33] A. MourA, Representations of the free profinite object over DA, Internat. J. Algebra Comput. 21(5) (2011), 675-701. DOI : 10.1142/S0218196711006467.

[34] W. PARry, On the $\beta$-expansions of real numbers, Acta Math. Acad. Sci. Hungar. 11(3-4) (1960), 401-416. DOI : 10.1007/BF02020954.

[35] D. Perrin And J.-É. Pin, "Infinite Words. Automata, Semigroups, Logic and Games", Pure and Applied Mathematics 141, Elsevier/Academic Press, The Netherlands, 2004.

[36] J.-É. PIN, Profinite methods in automata theory, in: "STACS 2009: 26th International Symposium on Theoretical Aspects of Computer Science", LIPIcs. Leibniz Int. Proc. Inform. 3, Schloss Dagstuhl. Leibniz-Zent. Inform., Wadern, 2009.

[37] J.-É. Pin AND P. WeIL, The wreath product principle for ordered semigroups, Comm. Algebra 30(12) (2002), 5677-5713. DOI : 10.1081/AGB-120016005.

[38] A. RÉNYI, Representations for real numbers and their ergodic properties, Acta Math. Acad. Sci. Hungar 8 (1957), 477-493. DOI : 10.1007/BF02020331.

[39] J. Rhodes and B. Steinberg, Profinite semigroups, varieties, expansions and the structure of relatively free profinite semigroups, Internat. J. Algebra Comput. 11(6) (2001), 627-672. DOI : 10.1142/S0218196701000784.

[40] J. Rhodes and B. Steinberg, "The q-theory of Finite Semigroups", Springer Monographs in Mathematics, Springer, New York, 2009. DOI : 10.1007/b104443.

[41] J. Rhodes and B. Tilson, The kernel of monoid morphisms, J. Pure Appl. Algebra 62(3) (1989), 227-268. DOI : 10.1016/0022-4049(89) 90137-0.

[42] J. G. Rosenstein, "Linear Orderings", Pure and Applied Mathematics 98, Academic Press, Inc. [Harcourt Brace Jovanovich, Publishers], New York-London, 1982.

[43] M. P. Schützenberger, On finite monoids having only trivial subgroups, Information and Control 8(2) (1965), 190-194. DOI: 10.1016/S0019-9958(65) 90108-7.

[44] H. Straubing, Aperiodic homomorphisms and the concatenation product of recognizable sets, J. Pure Appl. Algebra 15(3) (1979), 319-327. DOI: 10.1016/0022-4049(79)90025-2.

[45] W. Thomas, Languages, automata, and logic, in: "Handbook of Formal Languages", Vol. 3. Beyond Words, Springer, Berlin, 1997, pp. 389-455.

[46] B. Tilson, Categories as algebra: an essential ingredient in the theory of monoids, J. Pure Appl. Algebra 48(1-2) (1987), 83-198. DOI : 10.1016/00224049(87) 90108-3.

[47] P. WeIL, Profinite methods in semigroup theory. International Conference on Geometric and Combinatorial Methods in Group Theory and Semigroup Theory (Lincoln, NE, 2000), Internat. J. Algebra Comput. 12(1-2) (2002), 137-178. DOI : $10.1142 / \mathrm{S} 0218196702000912$.

[48] S. Willard, "General Topology", Addison-Wesley Publishing Co., Reading, Mass.-London-Don Mills, Ont. 1970. 
Jorge Almeida:

CMUP, Departamento de Matemática, Faculdade de Ciências, Universidade do Porto, Rua do Campo Alegre 687, 4169-007 Porto, Portugal

E-mail address: jalmeida@fc.up.pt

Alfredo Costa:

CMUC, Department of Mathematics, University of Coimbra, Apartado 3008, EC Santa Cruz, 3001-501 Coimbra, Portugal

E-mail address: amgc@mat.uc.pt

José Carlos Costa:

CMAT, Departamento de Matemática e Aplicações, Universidade do Minho, Campus de Gualtar, 4710-057 Braga, Portugal

E-mail address: jcosta@math.uminho.pt

Marc Zeitoun:

Université de Bordeaux, LaBRI, UMR 5800, F-33400 Talence, France

E-mail address: mz@labri.fr

Primera versió rebuda el 13 de març de 2017, darrera versió rebuda el 2 d'abril de 2019. 